\title{
QUANTIFICATION OF GOLD NANOPARTICLES IN BIOLOGICAL SAMPLES WITH TOTAL REFLECTION X-RAY FLUORESENCE
}

\author{
by \\ Gabriella Mankovskii \\ B.Sc., Ryerson University, 2015 \\ A thesis \\ presented to Ryerson University \\ in partial fulfilment of the \\ requirements for the degree of \\ Master of Science \\ in the program of \\ Biomedical Physics
}

Toronto, Ontario, Canada, 2017

(C) Gabriella Mankovskii, 2017 


\section{AUTHOR'S DECLARATION FOR ELECTRONIC SUBMISSION OF A THESIS}

I hereby declare that I am the sole author of this thesis. This is a true copy of the thesis, including any required final revisions, as accepted by my examiners.

I authorize Ryerson University to lend this thesis to other institutions or individuals for the purpose of scholarly research.

I further authorize Ryerson University to reproduce this thesis by photocopying or by other means, in total or in part, at the request of other institutions or individuals for the purpose of scholarly research.

I understand that my thesis may be made electronically available to the public.

GABRIELLA MANKOVSKII 


\title{
ABSTRACT
}

\section{QUANTIFICATION OF GOLD NANOPARTICLES IN BIOLOGICAL SAMPLES WITH TOTAL REFLECTION X-RAY FLUORESENCE}

\author{
Gabriella Mankovskii \\ Master of Science, Biomedical Physics \\ Department of Physics, Ryerson University, 2017
}

The applications of gold nanoparticles (AuNPs) in biomedicine span the imaging, diagnosis and therapy domains. Current methods of nanoparticle quantification are based on inductively coupled plasmas (ICP) methods, which are heavily influenced by sample preparation. This becomes challenging when quantifying trace-level amount of AuNPs in the presence of organic matrix. In this thesis, total reflection X-ray fluorescence (TXRF) spectrometry is proposed for quantifying cellular uptake of AuNPs in MDA-MB-231 breast cancer cell line suspensions. The most suitable internal standard and fitting approach that yield $90-110 \%$ recovery rate along with different sample preparation methods were investigated. Reference material AuNPs were used to validate the quantification capabilities of the newly developed method. Direct comparison of TXRF and ICP-AES showed ability of TXRF to accurately quantify nanoparticle uptake while further demonstrating the importance of sample preparation for ICP-AES. These results suggest that TXRF has potential for quantifying nanoparticle uptake and their kinetics. 


\section{ACKNOWLEDGMENTS}

This exciting and rewarding journey would not have been possible without the help of several individuals. I would like to thank a number of people who have contributed to the preparation and completion of this study.

It is with immense gratitude that I acknowledge the support and encouragement of my supervisor Prof. Ana Pejović-Milić. I have had the good fortune to work with Prof. Ana Pejović-Milić both as an undergraduate and graduate student. Throughout the years, I witnessed her inspiring creativity, drive and collaborative nature. I am very grateful for the opportunities and academic freedom she has given me. She provided insightful guidance throughout my thesis whilst giving me the freedom to explore my research interest. Her unique supervisory approach has made me a more confident researcher and taught me to appreciate the rigor and setbacks of a project. I am also very thankful for her support in attending international conferences in our discipline. The opportunity to present my work in front of a panel of experts has helped me grow as a researcher. Besides enriching my academic skills, Prof. Ana Pejović-Milić has also provided me with opportunities to build my soft skills. It has been an honor to be mentored by her.

I would also like to thank my supervisory committee members Dr. Raffi Karshafian and Dr. Carl Kumaradas. Their scientific input and line of questioning during our meetings strengthened this project and made me feel more prepared to defend my results. I appreciated their guidance as it taught me to be more skeptical of my data. Dr. James Gräfe is also gratefully acknowledged for his participation as an external member of my examination committee. I also want to recognize his offline assistance throughout my project and for providing me with lanthanum certified reference solution.

This thesis would not have been possible without the contributions of Dr. Devika Chithrani (University of Victoria) and her Ph.D. student Celina Yang. Under the guidance of Dr. Chithrani, Celina was solely responsible for making the gold nanoparticles used in this thesis and performing all the inductively coupled plasma measurements. I sincerely appreciate their willingness to provide me with nanoparticle and MDA-MB-231 samples. I am also very thankful 
for having the opportunity to engage in scientific discussions with both of them throughout this project.

I would also like to thank Diane Eichert for assisting me with PyMCA fitting. Despite her busy schedule, she always made time to address all my questions.

Prof. Peter Wobrauschek, Prof. Cristina Streli, and Prof. Tim Elam are also acknowledged for offering their expertise during the conferences I attended.

Matthew Forrest, Kevin Liu, Graham Ferrier and Arthur Worthington are recognized for their technical assistance. Sophia Finos' support with navigating the administrative aspects of the program is also very much appreciated. 
To my person 


\section{TABLE OF CONTENTS}

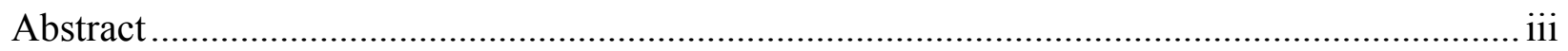

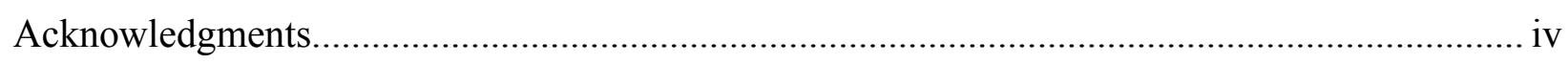

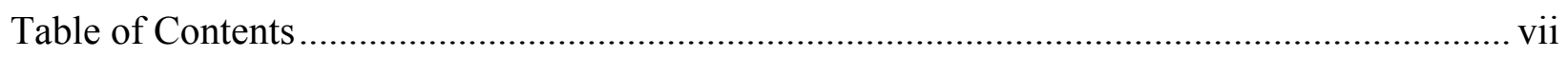

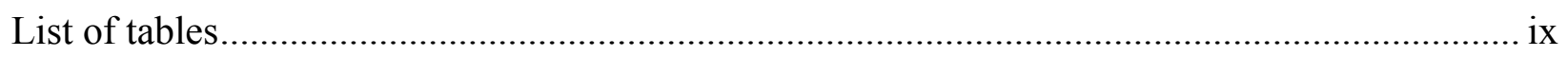

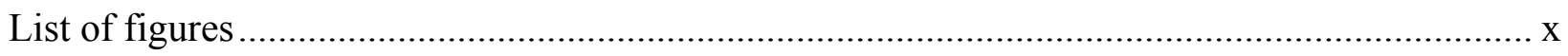

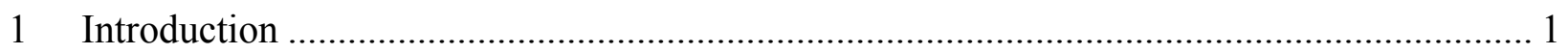

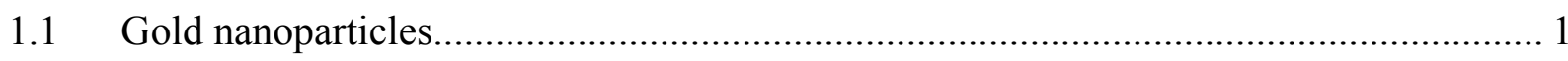

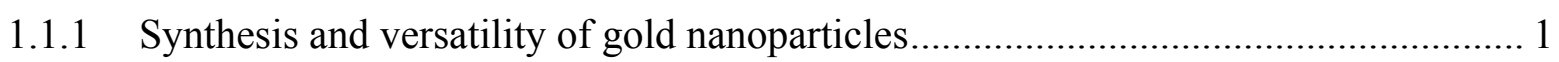

1.2 Biomedical applications of gold nanoparticles ........................................................ 3

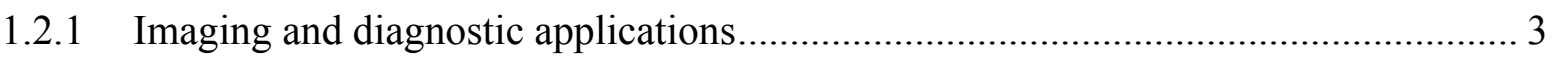

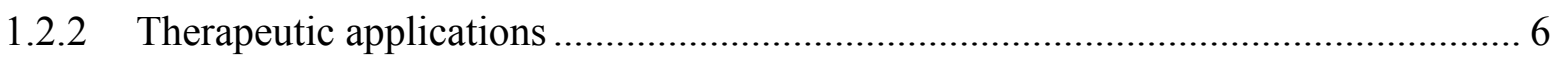

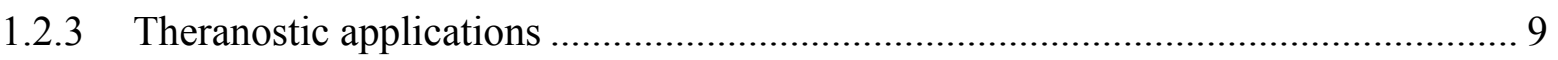

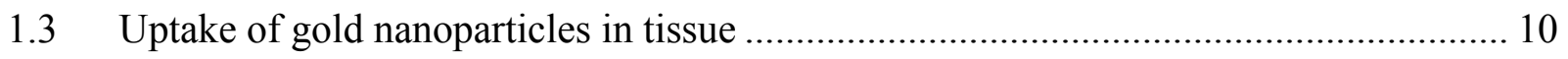

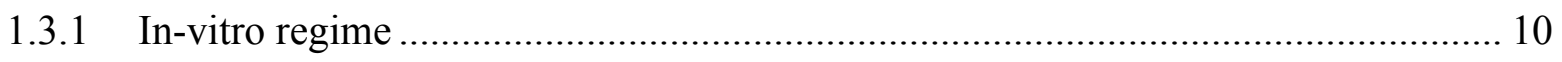

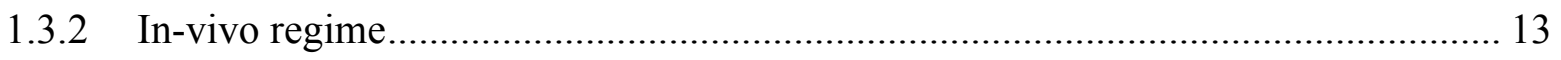

1.4 Quantitative methods of nanoparticle cellular uptake ................................................. 16

1.4.1 Inductively coupled plasma-based techniques.................................................... 17

$1.5 \quad$ Total reflection X-ray fluorescence spectrometry .................................................... 24

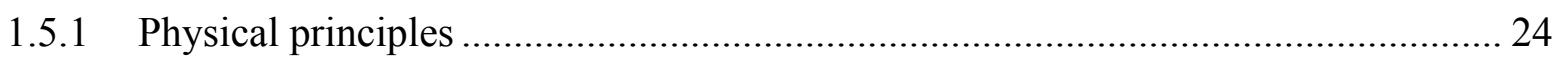

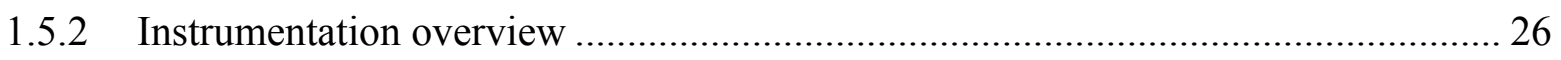

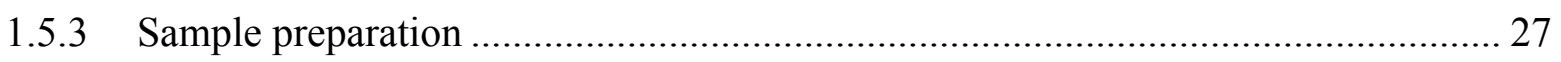

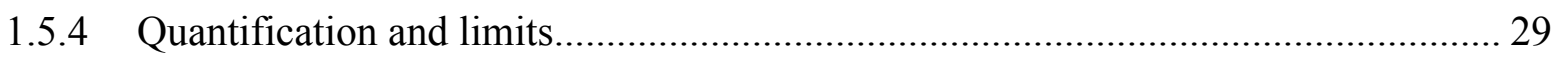

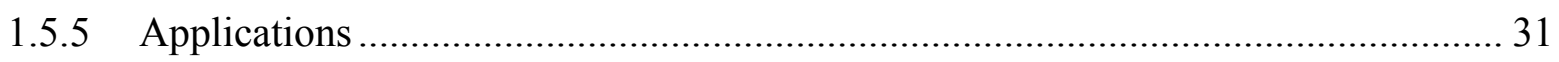

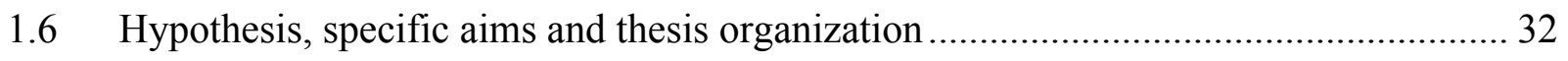

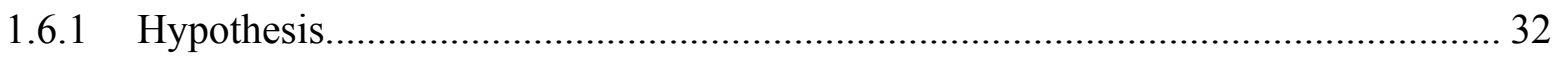

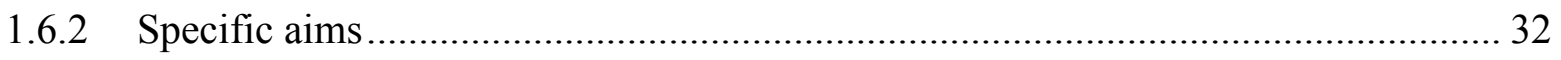

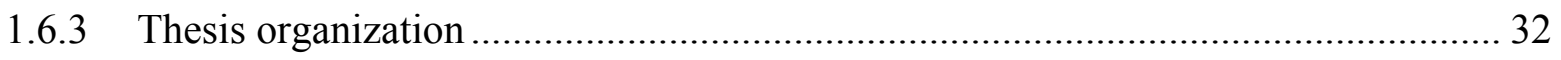

2 Total reflection X-Ray Fluorescence based quantification of gold nanoparticles in cancer

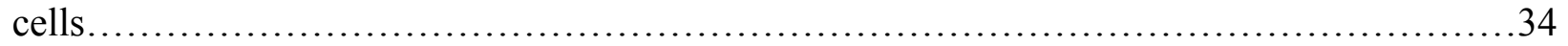

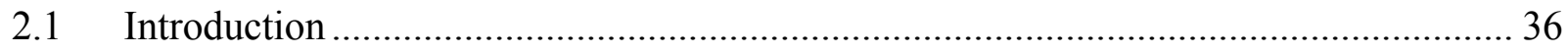

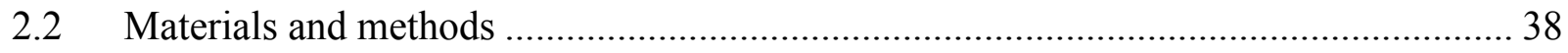

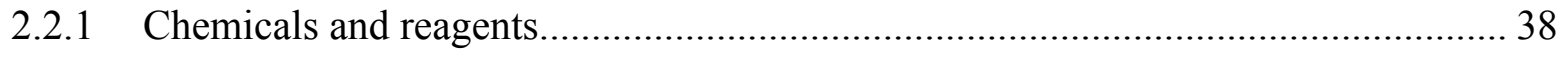

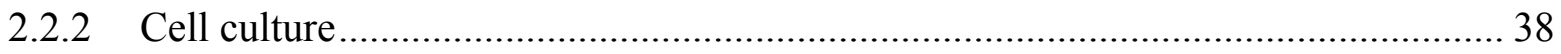


2.2.3 Sample preparation and measurement ............................................................ 39

2.2.4 Total reflection X-ray fluorescence spectrometer.............................................. 39

2.2.5 Spectral processing, calculations and statistical methods .................................. 40

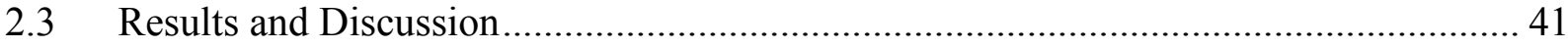

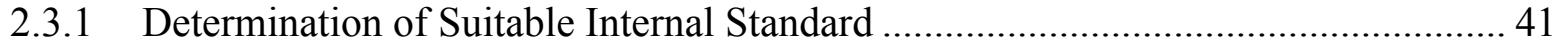

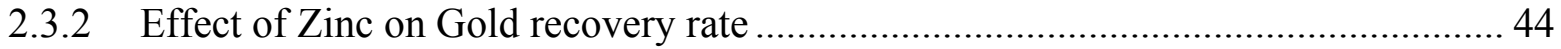

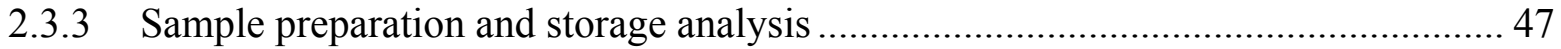

2.3.4 Recovery rate of reference material AuNPs .................................................... 50

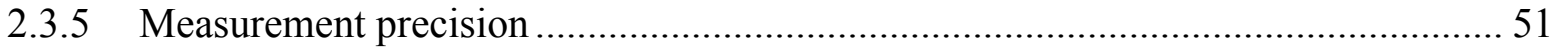

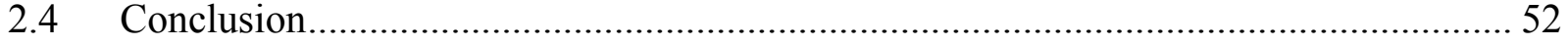

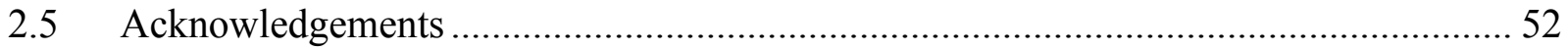

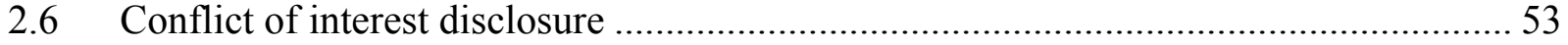

3 Comparison of TXRF quantification of gold nanoparticles with ICP-AES - preliminary

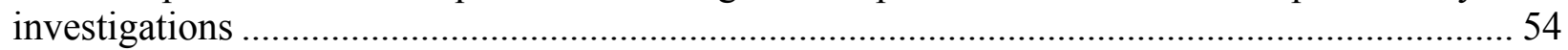

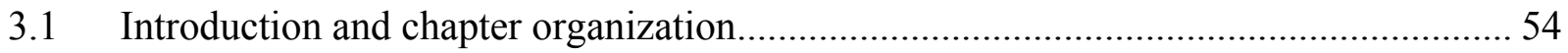

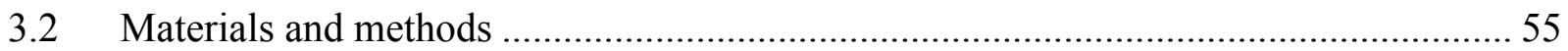

3.2.1 Cellular suspensions and nanoparticle synthesis ........................................... 55

3.2.2 ICP-AES: sample preparation and measurements ........................................... 57

3.2.3 TXRF: sample preparation and measurements ............................................... 58

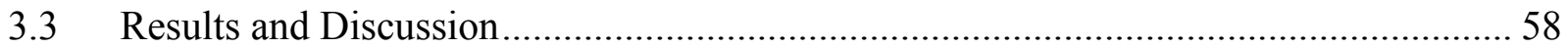

3.3.1 Recovery rates for reference material nanoparticles in presence of organic matrix. 58

3.3.2 ICP vs. TXRF: Comparisons in estimates of Au concentration ............................ 61

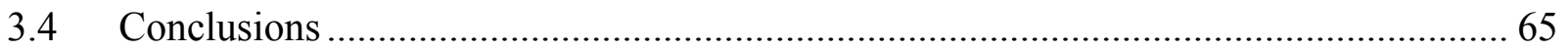

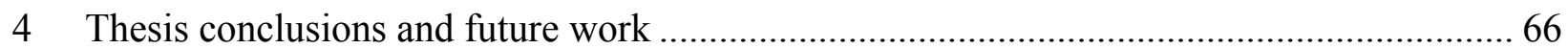

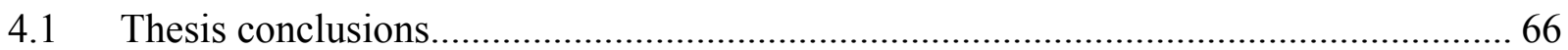

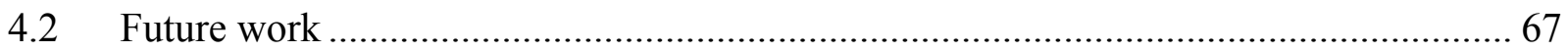

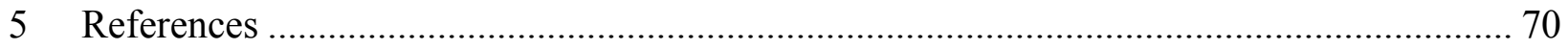




\section{LIST OF TABLES}

Table 2-1:Investigating the effect of sample position on measurement precision. Precision was evaluated with RSD\%. The results show obtained RSD\% for the 3 separate reflectors used per sample. Data was analyzed with PyMCA............................................................................ 51

Table 3-1: Summary of the samples measured with TXRF and ICP-AES.............................. 56 


\section{LIST OF FIGURES}

Figure 1-1: Versatility in the synthesis of AuNPs (adapted from [5]). (b) Optical absorption (given through extinction coefficient) spectrum of gold nanospheres (16 nm diameter), silver nanotriangles $(200 \mathrm{~nm}$ on edge) and gold nanorods $(10 \times 80 \mathrm{~nm})$ (adapted from [6]).................. 2

Figure 1-2: Light scattering images of anti-EGFR/gold (a) nanospheres and (b) nanorods after incubation for 30 minutes with oral cancer cells (adapted from [11]).

Figure 1-3: (a) Schematic of a lateral flow assay designed to detect the presence or absence of an analyte based on its interactions with GNPs (adapted from [14]). (b) Colorimetric detection of $\mathrm{Hg}^{2+}$ in urine based on mixing urine with gold nanoparticles and creatine/uric acid antibodies (adapted from [15]). (c) Nanoparticle-enabled dynamic light scattering assay designed to identify the presence of tumor-specific antigens in prostate cancer patients through binding to GNPs capped with citrate. The increase in the size of the GNP aggregates is used for staging the degree of the cancer (adapted from [16]).

Figure 1-4: (a) PA microscopy image of a subcutaneously inoculated B16 melanoma labelled with gold nanocages on the back of a living mouse (adapted from [18]). (b) Dual contrast ultrasound (gray) and photoacoustic (green) imaging of a tumor labelled with single-walled carbon nanotubes in a living mouse (adapted from [18]). (c) In-vivo ultrasound (gray) and photoacoustic (colors) imaging of mesynchemal stem cells labelled with gold nanotracers. The green cluster corresponds to the site of injection of the stem cells while the yellow strip is the melanin present in the skin of muscle of the hind limb of a living mouse (adapted from [19]).... 6

Figure 1-5: Scanning electron microscope of a macrophage cell exposed to a photosensitizer loaded nanoparticle. (a) After just 30 minutes of incubation, the nanoparticle (arrow) is adhered to the cell surface before entering (b) the cell through phagocytosis after 2 hours of incubation (adapted from [22]).

Figure 1-6: (a) In-vivo tumor volume as a function of treatment and days post-treatment. The irradiation was $30 \mathrm{~Gy}$ delivered via $250 \mathrm{kVp}$ photons (adapted from [26]). (b) In-vitro surviving fraction of HeLa cells irradiated with $220 \mathrm{kVp}$ photons for different (circle 1 x 109; triangle $1 \mathrm{x}$ $10^{8}$; square $1 \times 10^{7} \mathrm{AuNPs} / \mathrm{ml}$ concentrations of AuNPs (adapted from [28]) ............................. 8

Figure 1-7: Gold nanorods as theranostic agents for in-vivo X-ray computed tomography imaging and photothermal therapy. (a) Feasibility of the nanorods to be used as a diagnostic contrast agent for (b) computed tomography imaging. (c) Feasibility of the nanorods to be used a photothermal agent for (d) therapeutic increases in the temperature of a tumor. (e) Survival curves for PEGylated gold nanorods used in conjunction with laser irradiation (adapted from [29]).

Figure 1-8: Schematic of the mechanism of NP internalization inside a cell. (a) Larger particles are internalized through phagocytosis (b) Particles $>1 \mu \mathrm{m}$ can be nonspecifically internalized through micropinocytosis into generic vesicles. Smaller NPs can be internalized through several pathways, depending on size: (c) caveolar-mediated endocytosis for $60 \mathrm{~nm} \mathrm{NPs}$, (d) clatherin- 
mediated endocytosis for $120 \mathrm{~nm}$ NPs and (e) clatherin and caveolin-independent endocytosis for $90 \mathrm{~nm}$ NPs. Blue circles represent particles $>1 \mu \mathrm{m}$, blue stars about $120 \mathrm{~nm}$, red stars about $90 \mathrm{~nm}$ and yellow rods about $60 \mathrm{~nm}$ (adapted from [32]).

Figure 1-9: (a) Schematic showing the internalization of AuNPs through receptor mediated endocytosis (REM). The transport and processing of the particles inside the cell is also shown. (b) Size and (c) aspect ratio dependence on the cellular uptake. Transmission electron microscopy images showing (d) the internalization and (e) exocytosis of NPs from the cell (adapted from [36]).

Figure 1-10: (A) Schematic of a AuNP functionalized with peptides for nuclear targeting. (B) Trajectory of the peptide-conjugated AuNPs through the cell. Transmission electron microscopy images (C-F) showing the route AuNPs take in the cell from the cytoplasm (C), to organelles (D) followed by their escape from organelles $(\mathrm{E})$ and entering the nucleus through the nuclear pore complex (F). (G) Measurements of AuNPs excreted from the cell when conjugated with peptides. (H) Kinetics of the exocytosis process at 1 hour and 6 hours post-incubation (adapted from [27]).

Figure 1-11: (a) Schematic of the vasculature components inside a tumor showing the complex networks of vessels of varying sizes and shapes (adapted from [42]). (b) Realistic view of the active and passive targeting of tumors using nanoparticles (adapted from [43]).

Figure 1-12: Schematics of an ICP plasma torch (RF = radiofrequency; adapted from [52])..... 18

Figure 1-13: Schematic of an ICP-MS setup (MS = mass spectrometer; adapted from [55])..... 19

Figure 1-14: Schematic of an ICP-AES setup (adapted from [58]) ..................................... 21

Figure 1-15: (a) Approximate illustration of the working range of several atomic spectroscopy techniques and their capability of providing multielemental analysis (XRF $=\mathrm{X}$-Ray

Fluorescence, AAS = Atomic Absorption Spectroscopy, CVAAS = Cold Vapor AAS; adapted from [52]). (b) Features of ICP-MS and ICP-AES for the analysis of liquid samples (adapted from [53]).

Figure 1-16: Possible electron transitions in a heavy atom. The resulting characteristic X-rays and corresponding nomenclature are shown (adapted from [67])...................................... 25

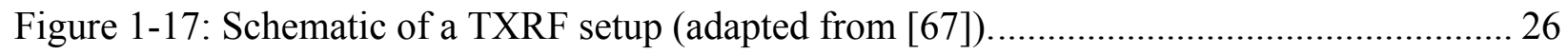

Figure 1-18: Schematic of internal standardization based sample preparation for TXRF analysis. The diagram shows the different steps involved in the process (adapted from [67])..... 30

Figure 2-1:Comparison of PyMCA, OriginPro and Bruker's fitting software. Results show recovery rate of $1 \mathrm{ppm}$ Au certified reference solution with $1 \mathrm{ppm} \mathrm{Y}$ as an internal standard using the original and newly calculated sensitivity factor of Au. The error bars represent the 
standard deviation of recovery rates from using 3 separate reflectors with 3 rotations per reflector.

Figure 2-2: Comparison of PyMCA, OriginPro and Bruker's fitting software. Results show recovery rate of $1 \mathrm{ppm}$ Au certified reference solution with $1 \mathrm{ppm}$ La as an internal standard using the newly calculated sensitivity factor of $\mathrm{Au}$. The error bars represent the standard deviation of recovery rates from using 3 separate reflectors with 3 rotations per reflector.

Figure 2-3: Evaluation of dependence of recovery rate on analyte concentration. Results show the recovery rate of $0.01 \mathrm{ppm}, 0.025 \mathrm{ppm}, 0.05 \mathrm{ppm}, 0.2 \mathrm{ppm}, 0.6 \mathrm{ppm}$ and $1 \mathrm{ppm}$ Au certified reference solutions with $1 \mathrm{ppm} \mathrm{La}$ as an internal standard using the newly calculated sensitivity factor of $\mathrm{Au}$. The error bars represent the standard deviation of recovery rates from using 3 separate reflectors with 3 rotations per reflector. Data was analyzed with PyMCA.

Figure 2-4: Representative TXRF spectrum of $1 \mathrm{ppm} \mathrm{Zn}$ and $1 \mathrm{ppm}$ Au certified reference solution. The spectrum shows spectral overlap of $\mathrm{Zn} \mathrm{K}_{\beta}$ and $\mathrm{Au} \mathrm{L}_{\alpha}$ peak.

Figure 2-5: Investigation of deconvolution efficacy of PyMCA, OriginPro and Bruker's fitting software with varying concentrations of $\mathrm{Zn}$. Results show the recovery rate of $1 \mathrm{ppm}$ Au certified reference solution with $0.5,1$ and $2 \mathrm{ppm} \mathrm{Zn}$ and $1 \mathrm{ppm}$ La as an internal standard using the newly calculated sensitivity factor of $\mathrm{Au}$. The error bars represent the standard deviation of recovery rates from using 3 separate reflectors with 3 rotations per reflector.

Figure 2-6: The effect of storage time and sample preparation methods on recovered $\mathrm{Au}$ concentrations. (A) In-house synthesized $10 \mathrm{~nm}$ AuNPs digested with 3:1 Aqua Regia and diluted with ultrapure water. (B) MDA-MB-231 cells incubated with In-house synthesized $10 \mathrm{~nm}$ AuNPs digested with 3:1 Aqua Regia and diluted with ultrapure water. The results show the obtained Au concentration with $1 \mathrm{ppm}$ La as the internal standard. The error bars represent the standard deviation of concentration from using 3 separate reflectors with 3 rotations per reflector. All data was analyzed with PyMCA

Figure 2-7: $10 \mathrm{~nm}$ reference material AuNPs digested with 4:1 Aqua Regia and $10 \mathrm{~nm}$ reference material AuNPs in the presence of suspensions of MDA-MD-231 cells digested with 4:1 Aqua Regia. The results show the obtained recovery rates with $1 \mathrm{ppm}$ La as the internal standard. The error bars represent the standard deviation of recovery rates from using 3 separate reflectors with 3 rotations per reflector. All data was analyzed with PyMCA. 50

Figure 3-1: Comparison of the TXRF and ICP-AES recovery rates of $10 \mathrm{~nm}$ NIST AuNPs in the presence of a suspension of MDA-MB-231 cells. The error bars for TXRF represent the standard deviation from recovery rates from using 3 separate cell suspensions with 3 separate reflectors each. The error bars for ICP-AES represent the standard deviation of triplicate measurements for 3 separate cell suspensions

Figure 3-2: Comparison of the estimated Au concentration from synthesized $10 \mathrm{~nm}$ AuNP suspensions. The error bars for TXRF represent the standard deviation from recovery rates from 
using 3 separate cell suspensions with 3 separate reflectors each. The error bars for ICP-AES represent the standard deviation of triplicate measurements for 3 separate cell suspensions.

Figure 3-3: Representative TXRF spectrum from MDA-MB-231 cells incubated with $10 \mathrm{~nm}$ AuNPs for 16 hours. The spectrum shows the relevant peaks of interest as a result of the X-ray tube (Mo), sample reflector ( $\mathrm{Si}$ ), internal standard (La) and the AuNPs overlapping with the endogenous $\mathrm{Zn}$ present inside cells

Figure 3-4: Comparative estimates of the number of AuNP per cell using TXRF and ICP-AES for boiled and un-boiled samples of MDA-MB-231 cells incubated with AuNPs. Incubation time was 16 hours. The error bars for TXRF represent the standard deviation from using 3 separate cell suspensions with 3 separate reflectors each. The error bars for ICP-AES represent the standard deviation of triplicate measurements for 3 separate cell suspensions. 


\section{INTRODUCTION}

\subsection{Gold nanoparticles}

The International Union of Pure and Applied Chemistry defines nanoparticles (NP) as particles which do not exceed $100 \mathrm{~nm}$ in any of the three dimensions [1]. Since the first description of gold nanoparticle (AuNP) synthesis in 1951 by Turkevich and colleagues [2], NP have undergone a revolution in terms of their applications in medicine and biology. This chapter will focus almost exclusively on AuNPs as they are the phenotype of nanoparticles that has experienced tremendous advancements in biomedicine.

\subsubsection{Synthesis and versatility of gold nanoparticles}

No universal method of synthesizing NPs exists due to the wide range of customization features. Turkevich's method relied in boiling chloroauric acid with small amounts of sodium citrate, a reducing and capping agent. This approach yields spherical AuNPs of about 10-20 nm in diameter. Frens developed a method in 1972 for preparing monodisperse suspensions of AuNPs whose size could be controlled in the range $1.5-150 \mathrm{~nm}$ by showing that the size of the gold nuclei formed at the start of the reduction depends on the amount of sodium citrate used [3]. Since then, AuNP synthesis has produced a myriad of shapes and sizes with versatile physical and optical properties. Figure 1-1a shows a schematic of the synthetic versatility of AuNPs. These particles allow for unique manipulation of the shape, size, surface coating and functionalization which overall enable precise fine tuning of particle properties. Another desirable characteristic of AuNPs is their optical tunability based on size, shape and surface coating as shown in Figure 1-1b. AuNPs and other nanoparticles exhibit surface plasmon resonance, a property where the conduction band electrons around the gold atoms oscillate in response to external electromagnetic radiation [4]. When the incident electromagnetic field matches the resonance frequency of the conduction band electrons, plasmon resonance is achieved and it explains why red-colored AuNP suspensions move towards the near infrared region as their size increases (Figure 1-1b). 
(a)

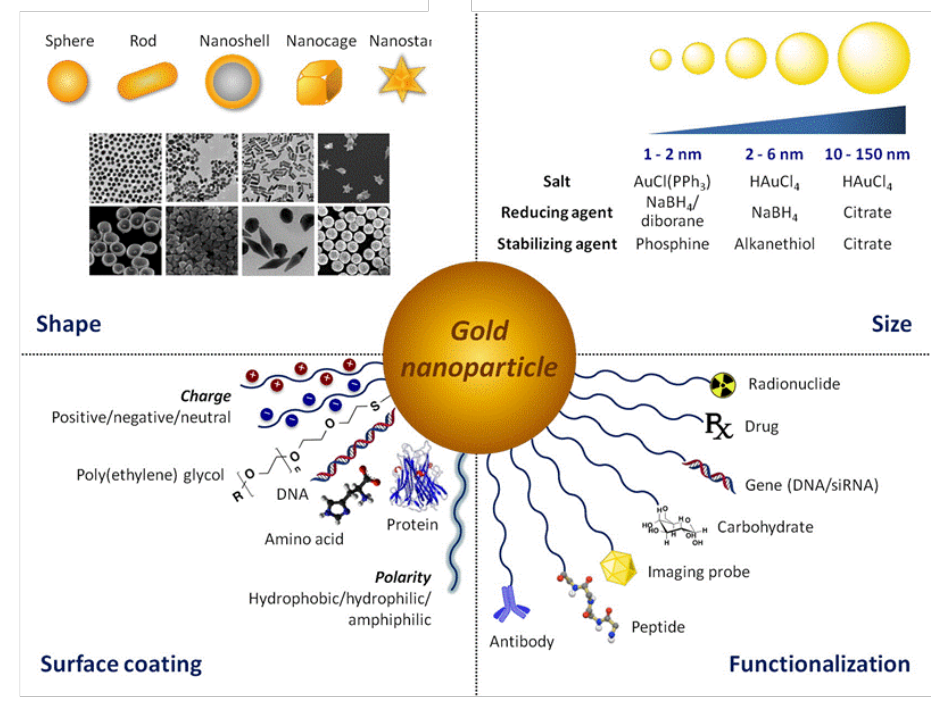

(b)

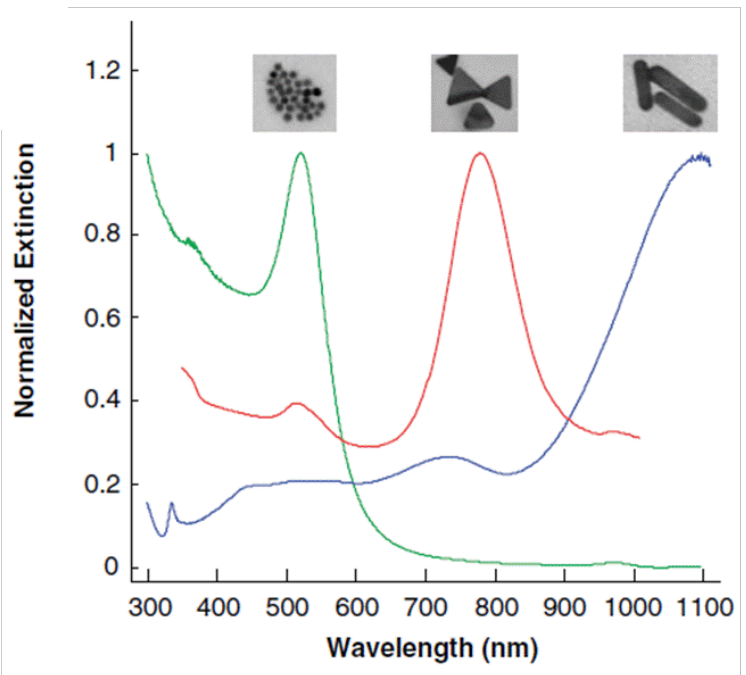

Figure 1-1: Versatility in the synthesis of AuNPs (adapted from [5]). (b) Optical absorption (given through extinction coefficient) spectrum of gold nanospheres (16 nm diameter), silver nanotriangles (200 $\mathrm{nm}$ on edge) and gold nanorods (10 x $80 \mathrm{~nm}$ ) (adapted from [6]).

Another property of AuNPs is their ability to bind to a number of materials and ligands which affect the aggregation of the nanoparticles. The choice of the ligand bound depends on the NP material, the solvent used, the size of the particle and of course the biomedical application of interest [7]. The binding of the ligands with the AuNP can be achieved via hydrophobic or electrostatic interactions, chemical absorption or through a head group (ex. thiol) on the ligand itself. The later will dictate the solubility of the NPs in various organic and inorganic solvents. Moreover, NP bioconjugation has been achieved with a wide range of molecules such as antibodies [8], sugars, lipids, vitamins, peptides and proteins [9] as well as DNA and RNA [10]. 
Figure 1-1a shows some schematics of the surface coating and functionalization that is possible using AuNPs. It is precisely the versatility of the aforementioned bioconjugation strategies which makes AuNPs attractive for a wide array of biomedical applications. Furthermore, the uptake and retention in biological tissues is directly influenced by the choice of the ligand as will be discussed in further detail in section 1.3.

\subsection{Biomedical applications of gold nanoparticles}

This section will present a brief summary of the most prevalent applications of AuNPs and other types of nanoparticles in imaging, therapy and recent efforts into creating theranostic capabilities within a single particle.

\subsubsection{Imaging and diagnostic applications}

\subsubsection{Microscopy, photoluminescence and assays}

Dark field microscopy has benefited significantly from the use of AuNPs. In its native state, the technique relies on the collection of the scattered light from the autoflourescence of cellular organelles upon illumination with a collimated light source. Conjugating the AuNPs with antiepidermal growth factors receptor (EGFR) antibodies provides not only additional contrast due to the strong scattering/absorption properties of the NPs but also specific binding [4]. Figure 1-2 shows representative light scattering images of anti-EGFR/AuNPs (spheres and rods) after being incubated for 30 minutes with various types of non-cancerous and malignant oral epithelial cells. It is obvious that the strong contrast provided by the presence of AuNPs allows for assessing the differences in binding between non-cancerous and malignant cells.

HaCaT nonmalignant cells

(a)

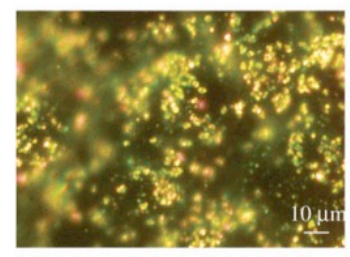

(b)

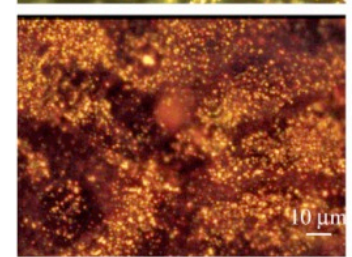

HSC malignant cells
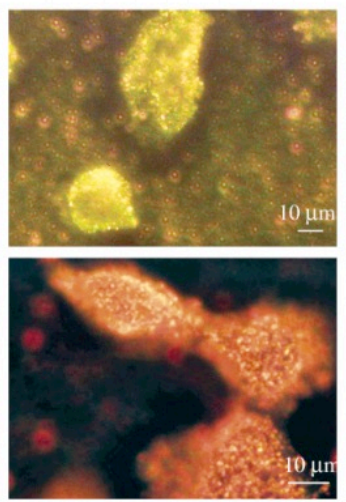

HOC malignant cells

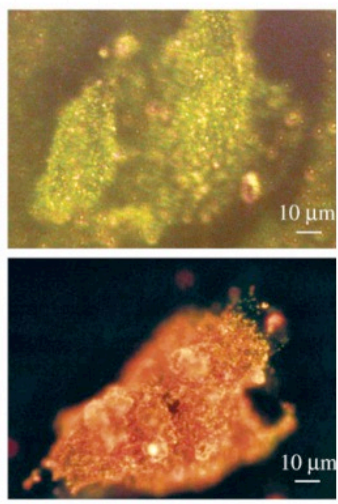

Figure 1-2: Light scattering images of anti-EGFR/gold (a) nanospheres and (b) nanorods after incubation for 30 minutes with oral cancer cells (adapted from [11]). 
AuNPs have also been able to contribute to photon-induced photoluminescence. The microscopy technique relies on the excitation of a fluorophore to an excited state using one or two photons in the UV or blue/green wavelengths. AuNPs by virtue of their strong optical absorption exhibit two or multi-photon luminescence in the near infra-red range [12]. This has enabled two-photon induced photoluminescence to use antibody-conjugated gold nanorods for 3D imaging of cancer cells in a depth up to $75 \mu \mathrm{m}$ due to the higher signal compared to tissue autoflouresence [13].

Beyond microscopy imaging techniques, NPs have also found use in diagnostic assays. In part due to the relative ease in NP surface modification, AuNPs provide a good foundation for biomolecule detection assays. Figure 1-3a shows a schematic of a generic lateral flow assay designed to detect the presence or absence of a target analyte using conjugations of antibodies with AuNPs [14]. More specifically, Du and colleagues developed a plasmonic colorimetric assay to detect the presence of mercuric ion in urine [15]. As shown in Figure 1-3b, the active components in urine (uric acid and creatine) were added to AuNPs to allow for the selective binding of the mercury ion which causes aggregation of the AuNPs ultimately leading to a color change due to surface plasmon resonance properties. Figure 1-3c shows another example where the size of the AuNP after binding with tumor-specific antigens is measured using a dynamic light scattering assay to screen for prostate cancer [16]. The human immunoglobin $\mathrm{G}$ ( $\operatorname{IgG})$ protein in the serum of prostate cancer patients is increased and along with other proteins in produces a larger corona around the AuNP which begins to aggregate with one another producing a metric of diagnosing prostate cancer. These recent examples provide a good overview of the sensitivity and specificity of AuNPs in being used as a diagnostic agent. 


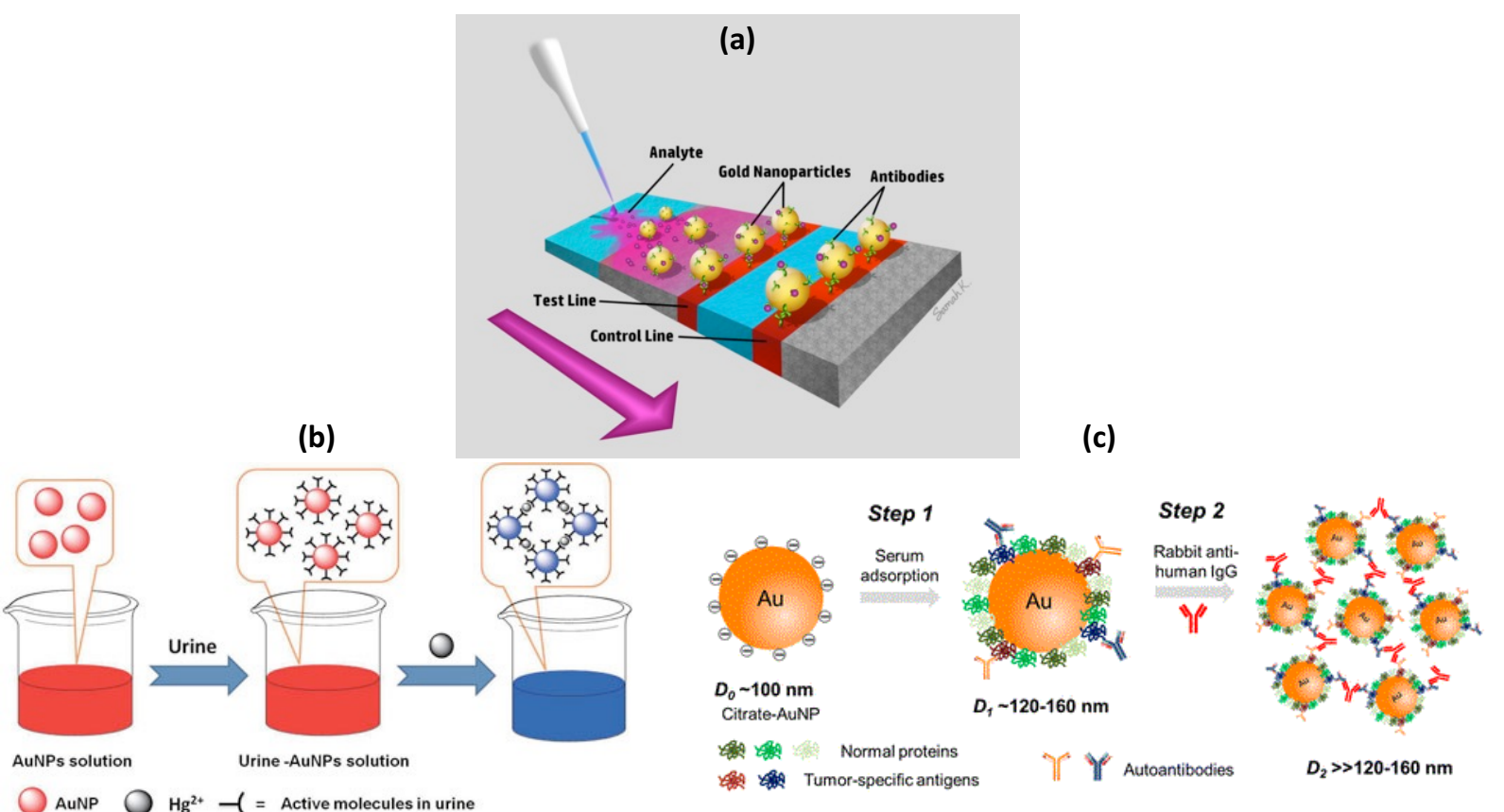

Figure 1-3: (a) Schematic of a lateral flow assay designed to detect the presence or absence of an analyte based on its interactions with GNPs (adapted from [14]). (b) Colorimetric detection of $\mathrm{Hg}^{2+}$ in urine based on mixing urine with gold nanoparticles and creatine/uric acid antibodies (adapted from [15]). (c) Nanoparticle-enabled dynamic light scattering assay designed to identify the presence of tumor-specific antigens in prostate cancer patients through binding to GNPs capped with citrate. The increase in the size of the GNP aggregates is used for staging the degree of the cancer (adapted from [16]).

\subsubsection{Photoacoustic imaging}

Owing due to their strong absorption in the near infrared regime (Figure 1-1b), AuNP have found ample utilization as contrast agents of a relatively newer imaging modality called photoacoustic (PA) imaging. This modality relies on absorption of electromagnetic radiation and the subsequent conversion of the absorbed energy into a mechanical sound wave. The optical absorption coefficient of AuNPs and other types of NPs is orders of magnitude higher than that of endogenous tissue chromophores such as hemoglobin or melanin and thus is an excellent candidate for the molecular tagging of tissues of interest [17]. Figure 1-4 shows examples of the ability of NP to enhance contrast of PA imaging in a variety of clinically relevant scenarios such as melanoma detection (Figure 1-4a), tumor labelling (Figure 1-4b) and stem cell differentiation (Figure 1-4c). 
(a)

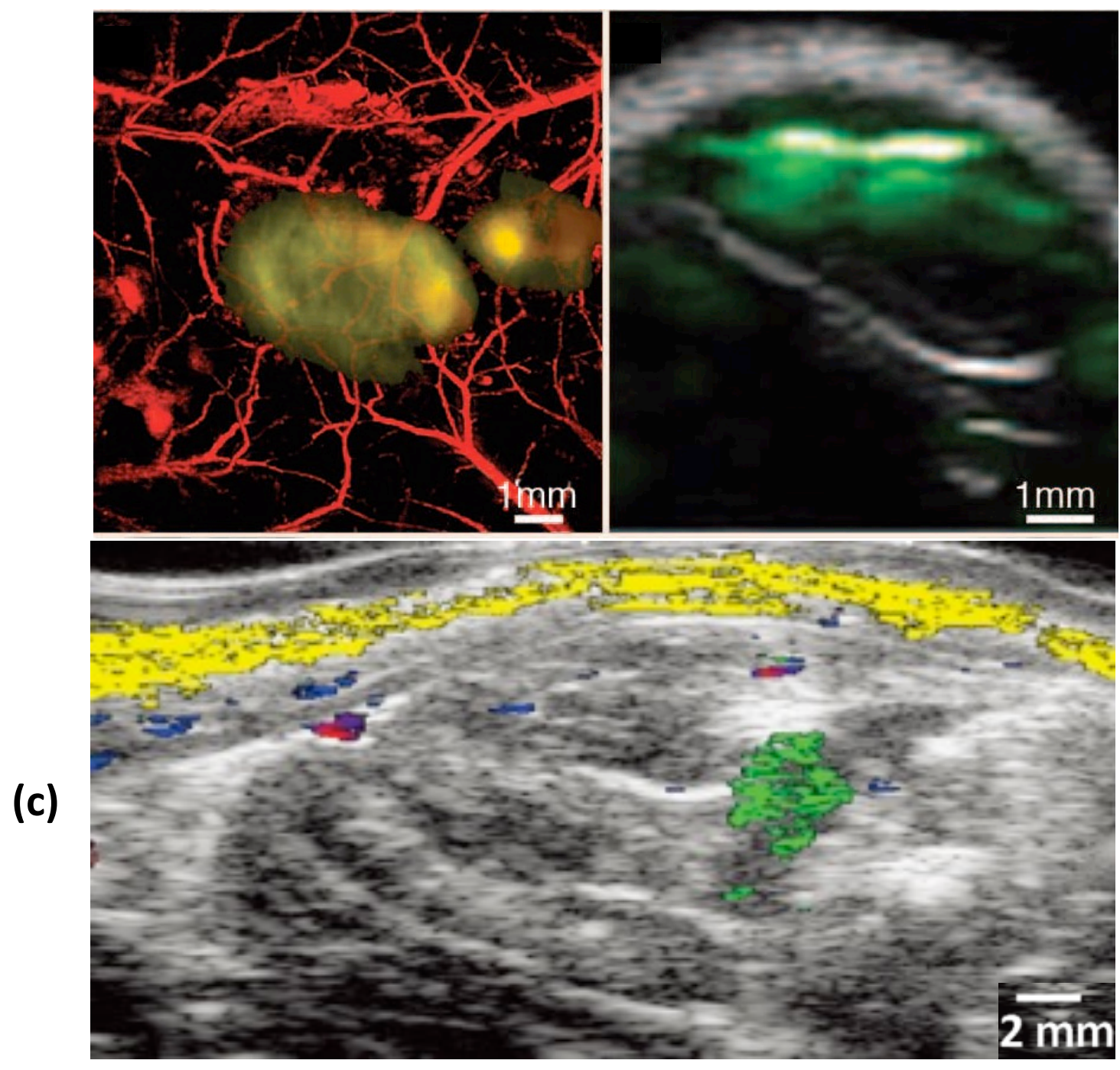

Figure 1-4: (a) PA microscopy image of a subcutaneously inoculated B16 melanoma labelled with gold nanocages on the back of a living mouse (adapted from [18]). (b) Dual contrast ultrasound (gray) and photoacoustic (green) imaging of a tumor labelled with single-walled carbon nanotubes in a living mouse (adapted from [18]). (c) In-vivo ultrasound (gray) and photoacoustic (colors) imaging of mesynchemal stem cells labelled with gold nanotracers. The green cluster corresponds to the site of injection of the stem cells while the yellow strip is the melanin present in the skin of muscle of the hind limb of a living mouse (adapted from [19]).

\subsubsection{Therapeutic applications}

\subsubsection{Photodynamic therapy}

As discussed in section 1.2.1.2, the optical absorption cross-section of NPs makes them ideal candidates for light based interactions. One of such interactions is photodynamic therapy (PDT) which relies on three key elements: light, a photosensitizer and molecular oxygen [20]. The 
photosensitizer is capable of producing reactive oxygen species (ROS) in collaboration with the inert, triplet state molecular oxygen found in tissue. The ROS produced are highly cytotoxic to cells and PDT has found numerous applications in cancer therapy [21]. The role of AuNP has emerged as that of carriers of the photosensitizer due to their versatility in surface modification. The latter also makes for easier targeting and localization of the photosensitizers inside cells as the AuNPs are readily phagocytosed within cells as early as 2 hours after incubation as shown in Figure 1-5 [22]. Another remarkable feat of NP use in PDT is that of up-conversion of light with longer wavelengths to shorter ones in the ultraviolet regime providing a more efficient photosensitizer absorption as well as lower toxicity [23].

(a)

(b)

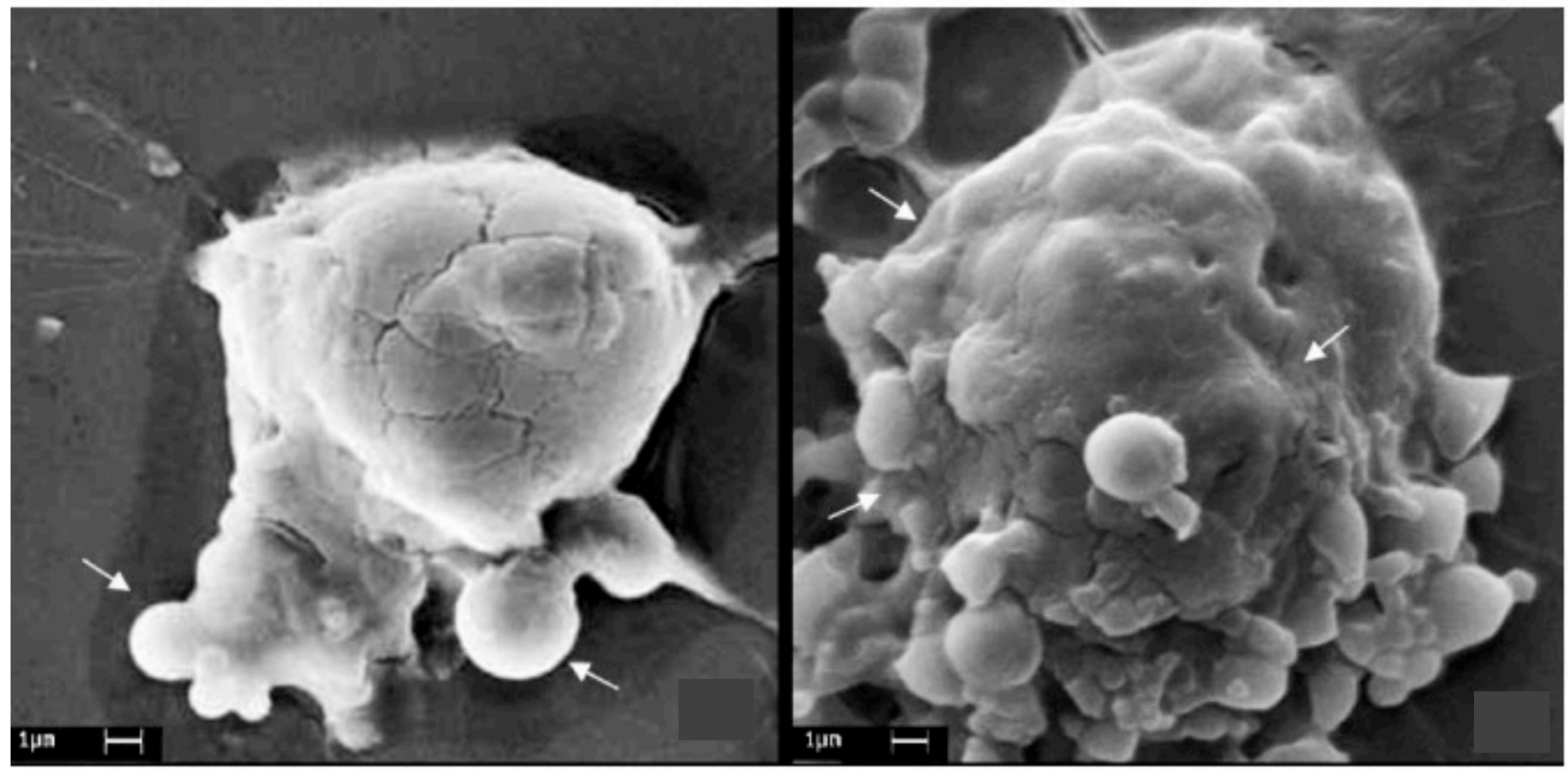

Figure 1-5: Scanning electron microscope of a macrophage cell exposed to a photosensitizer loaded nanoparticle. (a) After just 30 minutes of incubation, the nanoparticle (arrow) is adhered to the cell surface before entering (b) the cell through phagocytosis after 2 hours of incubation (adapted from [22]).

\subsubsection{Photothermal therapy}

Photothermal therapy is similar to PDT in that it too relies on the absorption of light. However, its goal is to exclusively convert the light into heat for the purposes of ablating tissue through coagulation of proteins [20]. Relying on the optical absorption peaks of AuNPs, many groups have shown a significant increase in temperature within a few minutes of irradiation time. In fact, Wang and colleagues demonstrated that there was nearly a 20 degree increase in 
temperature after irradiating a tumor inoculated with AuNPs for just 2 minutes [24]. They also found that gold nanohexapods had the highest cellular uptake, lowest cytotoxicity and highest photothermal conversion efficiency.

\subsubsection{Radiation therapy}

The NP-induced radiosensitization hypothesis was first proposed in the 1980s when iodine was used to sensitize mammalian cells in culture upon exposure to X-rays [25]. Since then, the role of high atomic number $(Z)$ elements for increasing the dose locally has been extensively studied. NP platforms in part due to their preferential accumulation in tumor tissue have been proposed as a good sensitizer of radiation therapy. AuNPs in particular have been extensively studied due to their high X-ray absorption coefficient as well as synthesis versatility. The seminal work of Hainfield et al in 2004 provided the first in-vivo evidence for the dose enhancement effects of AuNPs as shown in Figure 1-6a [26]. Chithrani and colleagues reported in-vitro enhancement factors of 1.43 at $220 \mathrm{kVp}$ and 1.17 at $6 \mathrm{MVp}$ photons for $50 \mathrm{~nm}$ AuNPs and demonstrated that this enhancement is directly dependent on the number of nanoparticles internalized within cells. The surviving fraction as a function of number of AuNPs per cell is shown in Figure 1-6b. Recent work on radiosensitization has revealed that nuclear tagging of AuNPs through peptide modifications improves cellular uptake, and intracellular retention suggesting that better radiosensitization approaches are indeed possible [27].

(a)

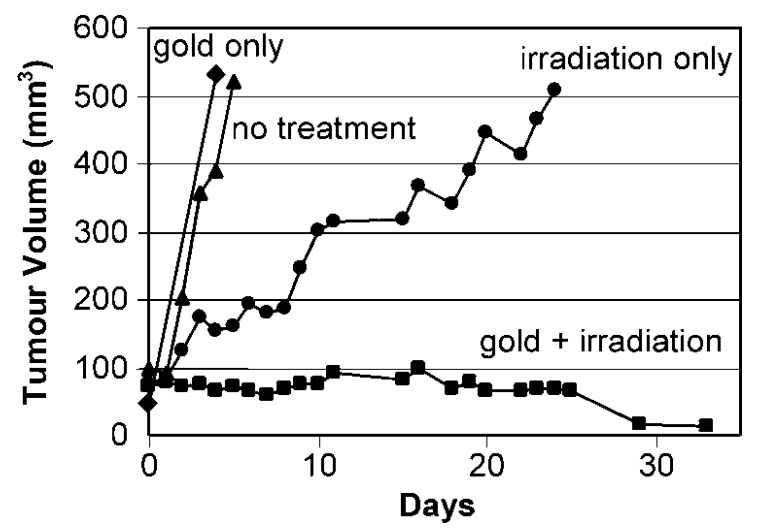

(b)

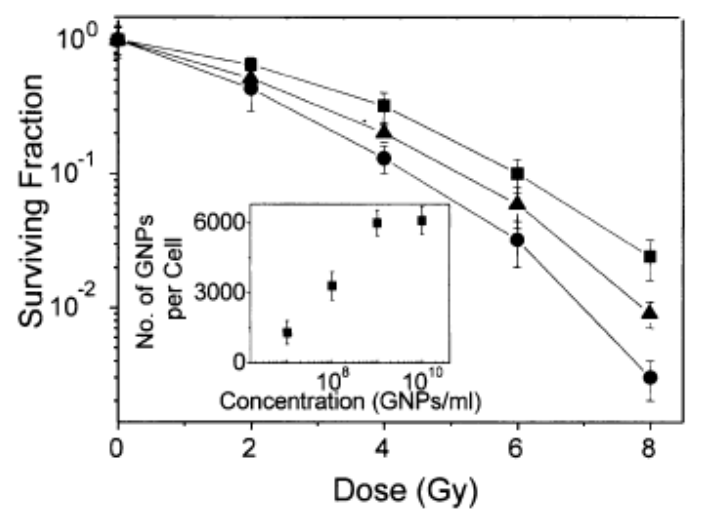

Figure 1-6: (a) In-vivo tumor volume as a function of treatment and days post-treatment. The irradiation was 30 Gy delivered via $250 \mathrm{kVp}$ photons (adapted from [26]). (b) In-vitro surviving fraction of HeLa cells irradiated with 220 $\mathrm{kVp}$ photons for different (circle $1 \times 10^{9}$; triangle $1 \times 10^{8}$; square $1 \times 10^{7}$ AuNPs/ml concentrations of AuNPs (adapted from [28]). 


\subsubsection{Theranostic applications}

Theranostic agents have been recently proposed as a vehicle that will simultaneously deliver imaging and therapeutic agents for combined diagnosis and therapy. Figure 1-7 shows how AuNP can be used as a theranostic agent for in-vivo X-ray computed tomography imaging as well as for photothermal therapy [29]. The presence of the gold nanorods clearly demarcates the location of the tumor in Figure 1-7b and the oscillation of the electron cloud in Figure 1-7c is sufficient to increase the temperature in the tumor by nearly 40 degrees in a localized manner (Figure 1-7d). Most importantly, the PEGylated nanorods irradiated with a laser beam had greater survival rates than the control group as shown in Figure 1-7e. Cheng and colleagues developed $\mathrm{Fe}_{3} \mathrm{O}_{4}$ and quantum dot-conjugated taxol-loaded PLGA nanoparticles onto which poly(styrenesulfonate) gold nanorods were attached [30]. These multifunctional nanoparticles could be used for chemotherapy and near infrared photothermal ablation of cancer cells. MRIcompatible theranostic agents have also been developed with magnetic nanoparticles carrying chemotherapeutic drugs [31]. These results clearly indicate that NPs have a lot of potential in being used as a multifunctional diagnostic and therapeutic particle.

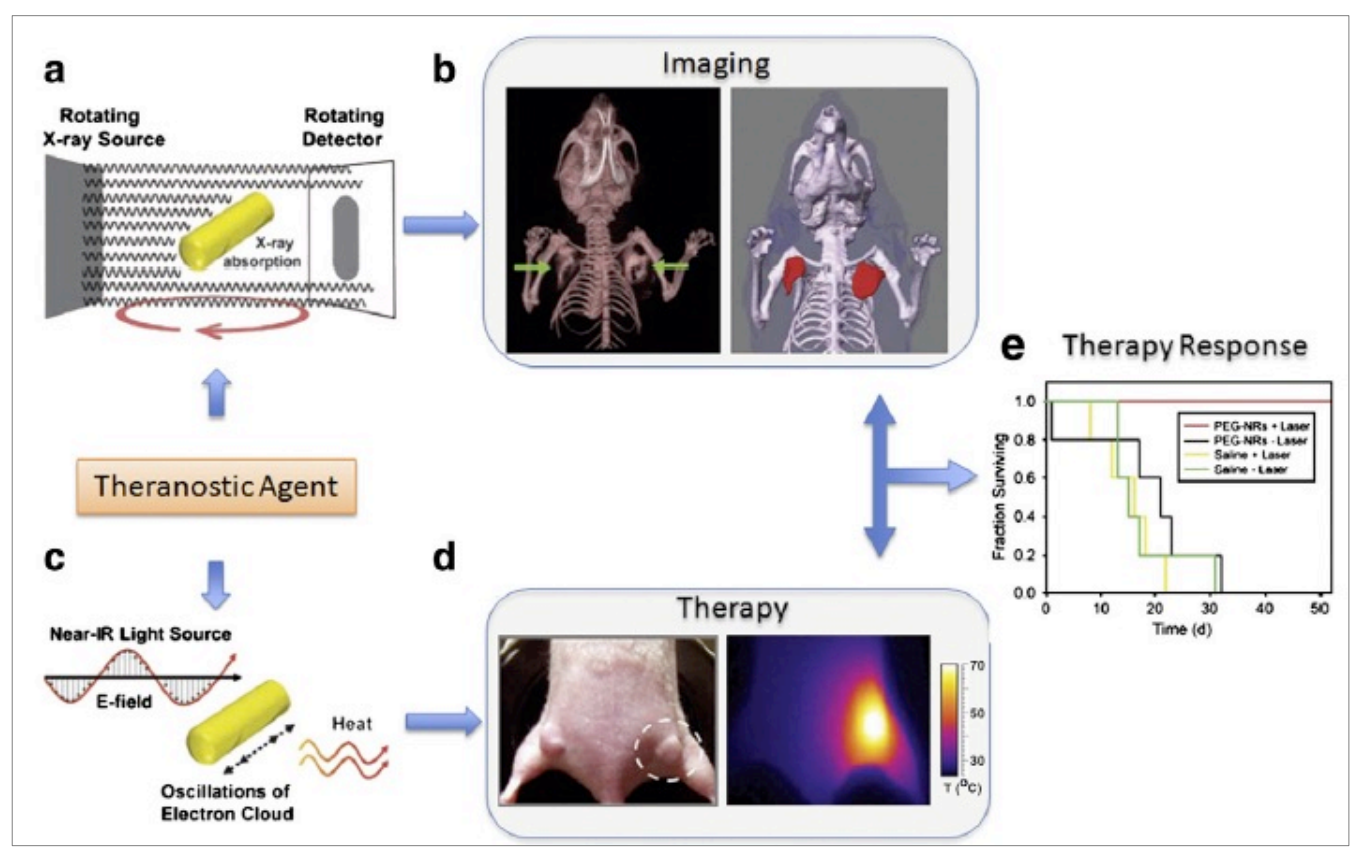

Figure 1-7: Gold nanorods as theranostic agents for in-vivo X-ray computed tomography imaging and photothermal therapy. (a) Feasibility of the nanorods to be used as a diagnostic contrast agent for (b) computed tomography imaging. (c) Feasibility of the nanorods to be used a photothermal agent for (d) therapeutic increases in the temperature of a tumor. (e) Survival curves for PEGylated gold nanorods used in conjunction with laser irradiation (adapted from [29]). 


\subsection{Uptake of gold nanoparticles in tissue}

This section will discuss the strategies and mechanisms in place for understanding the means through which NPs enter and leave cells. For all the applications discussed in section 1.2, it is necessary to manipulate with a high degree of accuracy the accumulation, biodistribution and time kinetics of NPs inside cells. The in-vitro regime will be first discussed to show the proof-ofprinciple experiments that have elucidated the mechanisms for cellular uptake and removal. Invivo studies, particularly pertaining to NP extravasation within tumor vasculature will be then summarized to show the feasibility of in-vitro concepts. Finally, a brief discussion of the challenges and opportunities facing the translation of these ideas will be provided.

\subsubsection{In-vitro regime}

The modes for the internalization of NPs based on their respective size limitations are shown in Figure 1-8 [32]. Several biological barriers at a cellular level must be overcome by NPs prior to entering the cell. The first barrier is the cellular membrane which blocks the diffusion of complexes greater than $1 \mathrm{kDa}$. This physical barrier is overcome by endocytosis whose exact mode depends on the size of the particle as well as the destination of the NP once inside the cell. For instance, clatherin-mediated endocytosed particles are destined for a lysosomal compartment while the caveolin-mediated ones are not [33]. Endosomal escape in clatherin-mediated endocytosis must occur prior to fusion with a lysosome in order to prevent the destruction of the cargo under the harsh, acidic lysosomal conditions [34]. In both cases, endosomal escape is required to access the desired subcellular compartment (mitochondria, nucleus or cytosol) [35]. 


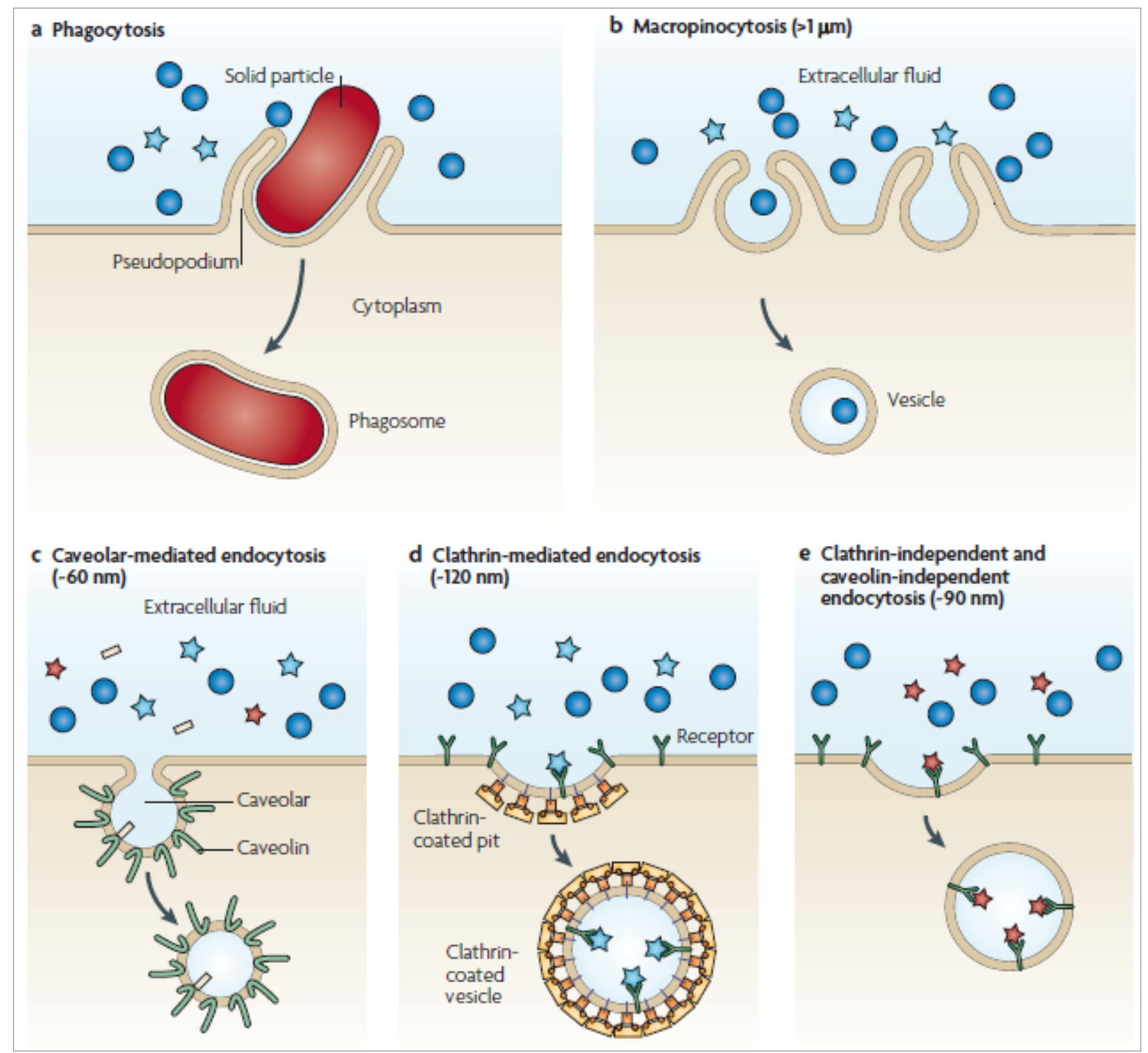

Figure 1-8: Schematic of the mechanism of NP internalization inside a cell. (a) Larger particles are internalized through phagocytosis (b) Particles $>1 \mu \mathrm{m}$ can be nonspecifically internalized through micropinocytosis into generic vesicles. Smaller NPs can be internalized through several pathways, depending on size: (c) caveolar-mediated endocytosis for $60 \mathrm{~nm} \mathrm{NPs,} \mathrm{(d)} \mathrm{clatherin-mediated} \mathrm{endocytosis} \mathrm{for} 120 \mathrm{~nm}$ NPs and (e) clatherin and caveolinindependent endocytosis for $90 \mathrm{~nm}$ NPs. Blue circles represent particles $>1 \mu \mathrm{m}$, blue stars about $120 \mathrm{~nm}$, red stars about $90 \mathrm{~nm}$ and yellow rods about $60 \mathrm{~nm}$ (adapted from [32]).

The conjugation of the surface of NPs with ligands has been shown to influence the mode of cellular internalization. Folic acid, albumin and cholesterol facilitate uptake through caveolinmediated endocytosis while glycoreceptors prefer the clatherin pathway [32], [33]. The remaining of the internalization pathways seems to be promoted by the use of membrane penetrating peptides. 
Figure 1-9 shows another schematic of the internalization process of NPs focusing more on the fate of the particle once inside the cell and the cellular excretion of the particles [36]. The fusing of the endosomes with the lysosomes after the receptor mediated endocytosis (REM) process is shown in detail in Figure 1-9a. This process is shown to be dependent on the size of the NPs as shown in Figure 1-9b and Figure 1-9c where both the diameter of the AuNPs and the aspect ratio seem to strongly influence the number of particles internalized within the cell. Figure 1-9d shows transmission electron microscopy images providing evidence for the encapsulation of NPs inside lysosomes. The excretion of NPs from cells takes place through the exocytosis process as shown in Figure 1-9e. Much like endocytosis, this process is also energydependent. Interestingly, the exocytosis process is very quick with almost $65 \%$ of the internalized NPs undergoing extraction within 30-40 minutes of the removal of an extracellular concentration gradient [34]. Furthermore, it has been shown that the exocytosis of gold nanorods is remarkably faster than spherical counterparts. This has been attributed to the fewer number of ligands bound to the surface of rods compared to spheres [34], [36], [37].

(a)

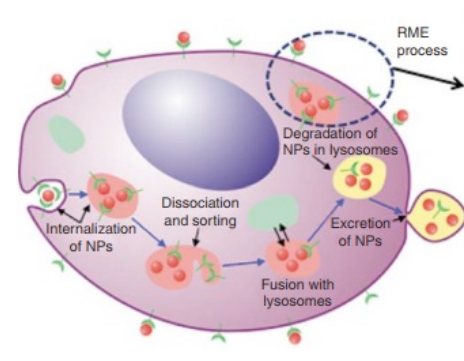

(b)

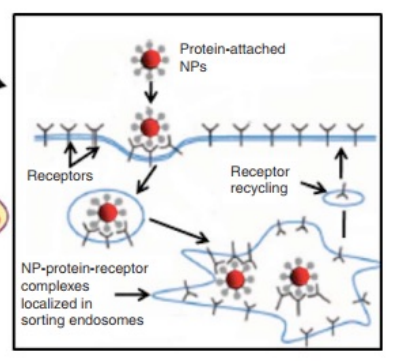

(c)
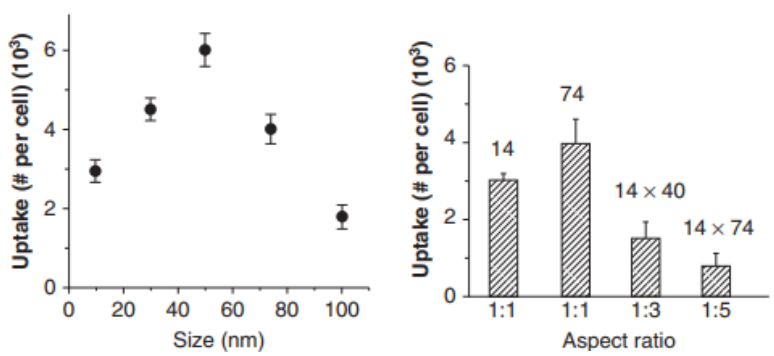

(d)
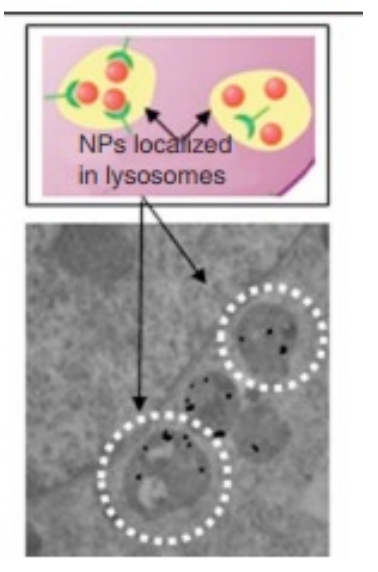

(e)
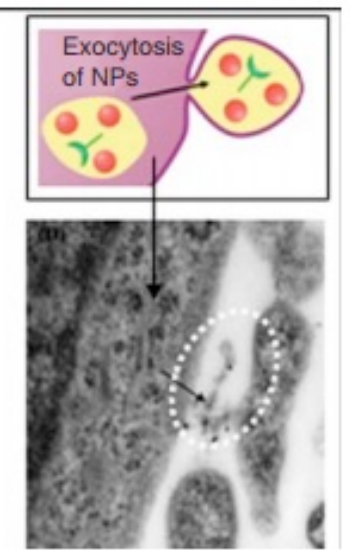

Figure 1-9: (a) Schematic showing the internalization of AuNPs through receptor mediated endocytosis (REM). The transport and processing of the particles inside the cell is also shown. (b) Size and (c) aspect ratio dependence on the cellular uptake. Transmission electron microscopy images showing (d) the internalization and (e) exocytosis of NPs from the cell (adapted from [36]). 
It is important to note that in the internalization discussion thus far, the nucleus and cytoplasm of the cell remain NP free when the endocytosis/lysosomal pathways are employed. Recent studies have shown that targeting the nucleus of the cell with NPs enhances the therapeutic effects not only for radiosensitization purposes but also for gene therapy [9], [38][41]. Figure 1-10 shows the effect that nuclear targeting with peptides has on the ability to localize AuNPs into the nucleus [27]. As shown in Figure 1-10b, nuclear-targeted AuNPs escape the endosomal pathway which would otherwise excrete them from the cell and instead interact with the nuclear pore complex (Figure 1-10f). This ensures internalization into the nucleus and about 2x fewer exocytosed particles compared to unmodified AuNPs (Figure 1-10g). Overall, there is about $1.5 \times 10^{4}$ AuNPs per cell at 1 hour and 6 hours post-incubation when conjugating with peptides compared to approximately $5 \times 10^{3}$ when unmodified AuNPs are used (Figure 1-10h). These encouraging results suggest that AuNP targeting of the nucleus can be achieved with reasonable success thus creating new therapeutic avenues.
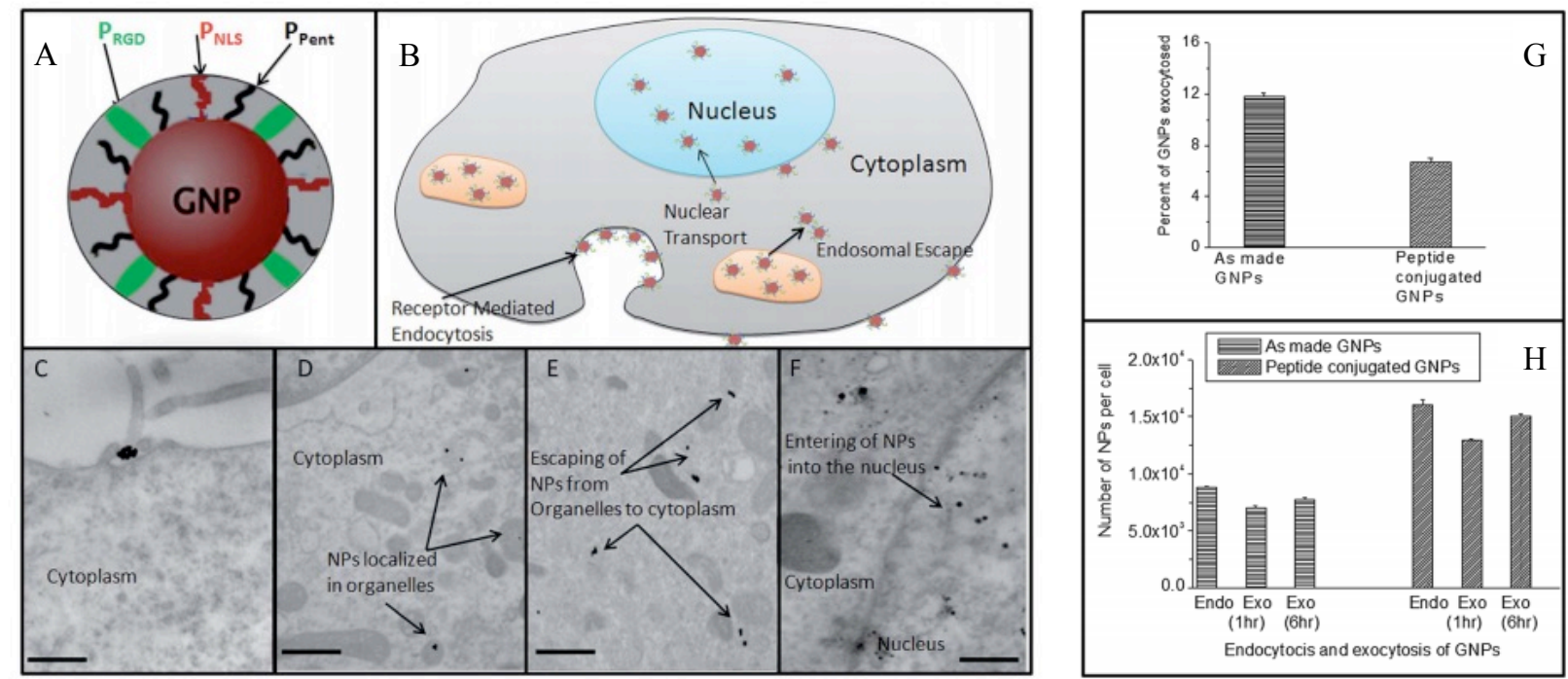

Figure 1-10: (A) Schematic of a AuNP functionalized with peptides for nuclear targeting. (B) Trajectory of the peptide-conjugated AuNPs through the cell. Transmission electron microscopy images (C-F) showing the route AuNPs take in the cell from the cytoplasm (C), to organelles (D) followed by their escape from organelles (E) and entering the nucleus through the nuclear pore complex (F). (G) Measurements of AuNPs excreted from the cell when conjugated with peptides. $(\mathrm{H})$ Kinetics of the exocytosis process at 1 hour and 6 hours post-incubation (adapted from [27]).

\subsubsection{In-vivo regime}

The achievements summarized in section 1.3.1 highlight the evolution of nanotechnology in tackling some of the most relevant problems in medicine today. However, they present only half 
of the monumental challenge of delivering the nanoparticles into the tissue of interest under invivo conditions. Given that one of the most investigated applications of NPs is tumor targeting, the rest of this section will focus on the successes, challenges and opportunities present in achieving delivery of a desirable dose.

A schematic of the tumor microenvironment with special emphasis on the challenges that NPs face as they circulate through the chaotic vasculature present in tumors is shown in Figure 1-11 [42]. It is immediately striking that the structural organization of tumor blood vessels differs from that of normal tissue. Mother vessels (Figure 1-11a) are characterized by thinned or compressed endothelial cells and as a result are highly permeable to small molecules and larger blood plasma proteins. These vessels are typically unstable and over time differentiate into other vascular malformations which give rise to larger, tortuous blood vessels which supply and drain angiogenic vasculature. 


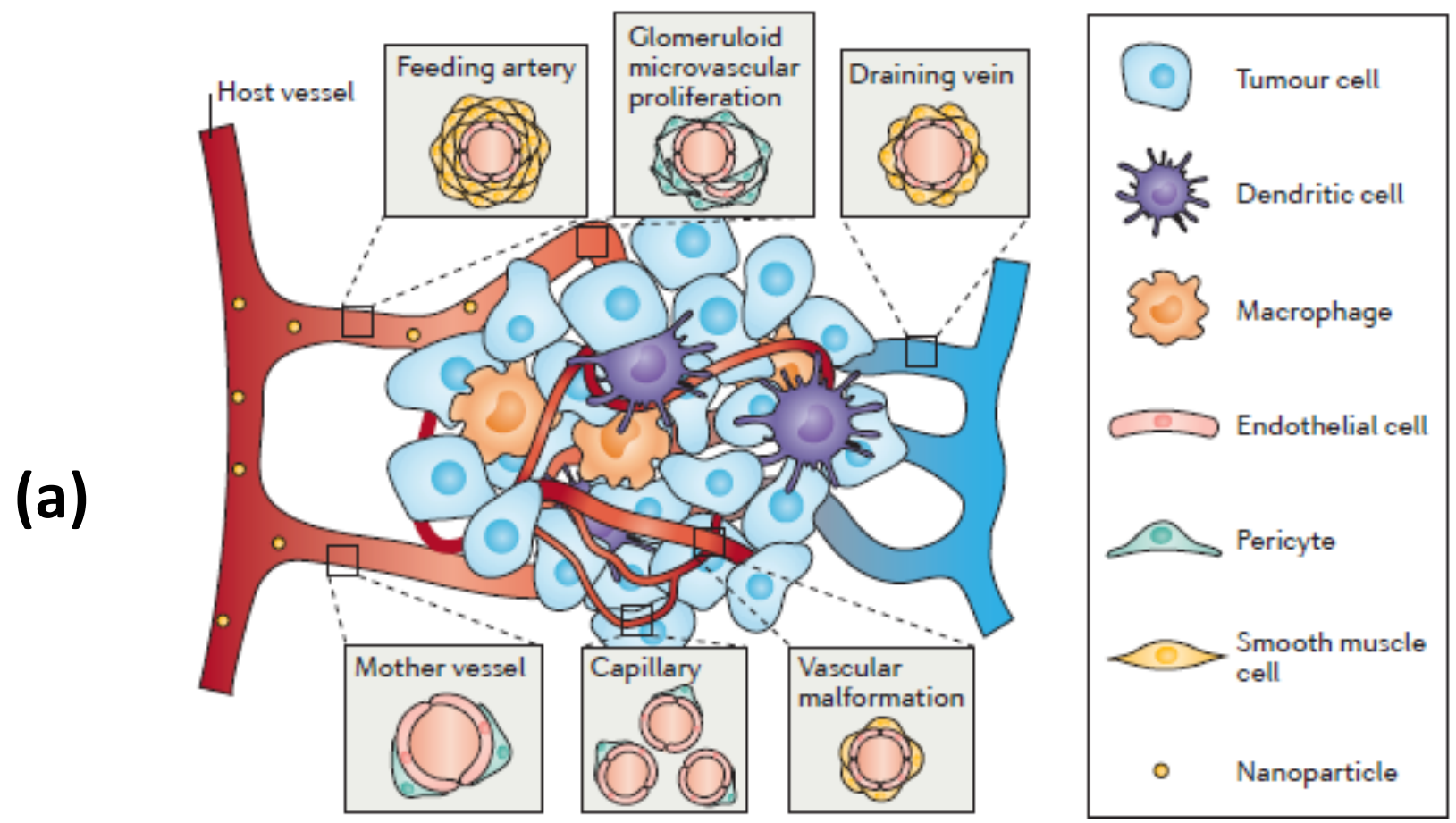

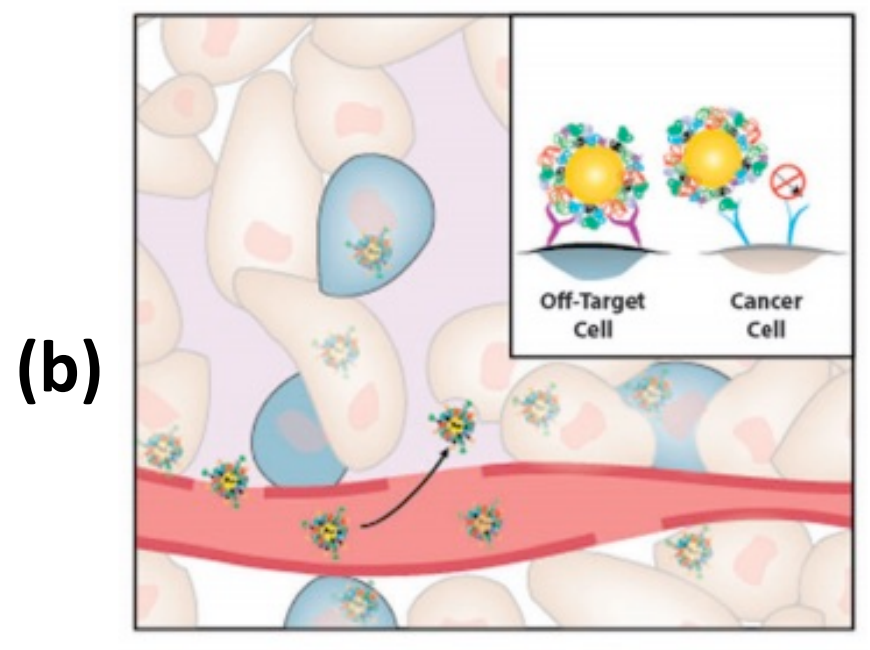

Active Targeting

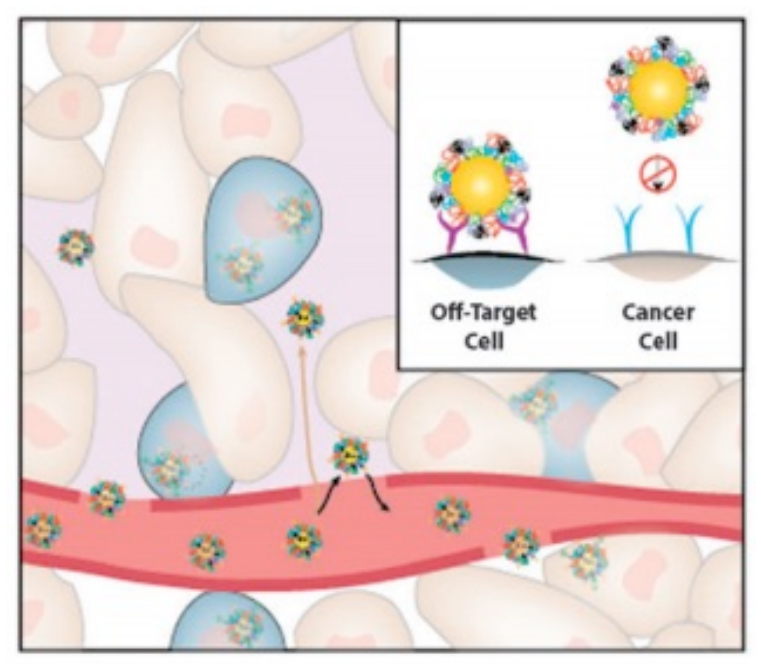

Passive Targeting

Figure 1-11: (a) Schematic of the vasculature components inside a tumor showing the complex networks of vessels of varying sizes and shapes (adapted from [42]). (b) Realistic view of the active and passive targeting of tumors using nanoparticles (adapted from [43]).

It is reported in literature that limited, in-vivo therapeutic efficacy across many nanoparticle formulations is attributed to the complexities of the tumor vasculature. Typically, less than $10 \%$ of systemically administered nanoparticles accumulate within the tumor and an even smaller number reach the inside of tumor cells [43]-[46]. As shown in Figure 1-11b, the transport of NPs from the tumor vasculature to the target tissue of interest can be achieved 
through active or passive targeting of the NPs [43]. Passive targeting relies on the enhanced permeability and retention (EPR) effect which suggests that if the particles are small enough, they will extravasate into the tumors through pores $(\sim 200 \mathrm{~nm})$ created in the blood vessels during the vascularization process shown in Figure 1-11a. This process is however limited by the diffusion of the NP into and out of the tumor. In active targeting, the NP is coated with molecules or ligands which theoretically will allow the particle to bind to the endothelial cells, to the extracellular matrix or to the cancer cell itself. This idealized method is often affected by the presence of a protein corona at the NP surface which will cause new interactions with off-target cells and deviate the NP from its intended target [49]. Moreover, passive targeted formulations tend to have longer half-lives because of the lack of protein serum interactions which prevents NPs from being recognized by the macrophages [47]. In additional to the vascular-induced challenges described, the poor retention of NPs within the tumor space is also affected by uptake in the skin, spleen and liver.

The challenges revolving around targeted delivery of NPs in tumor tissues are eloquently summarized in a recent study by Warren Chen's team [42]. They concluded from surveying the literature of the past 10 years, only $0.7 \%$ of the administered NP dose is found to be delivered to a solid tumor, a disappointing fact with negative consequences in the clinical translation of nanomedicines. In supporting these findings, Sykes and colleagues have quantitatively shown that the tumor pathophysiology and volume can significantly affect nanoparticle targeting [48]. Their analysis presents a paradigm shift in nanomedicine which enables researchers from trying to develop a "one-size-fits-all" approach in NP development. Instead, the authors encourage researchers to develop NPs according to tumor characteristics of each patient. This is a monumental task which will most certainly require novel means of assessing the efficacy of NP delivery in tumor tissue. This thesis addresses the challenge of quantitative assessments of NP concentration in cancerous tissue.

\subsection{Quantitative methods of nanoparticle cellular uptake}

All the applications described in section 1.2 require rigorous methods of assessing the concentration of NPs inside cells and eventually in biological tissue. This portion of the introduction will describe two methods of quantification, namely inductively coupled plasmabased techniques, an established spectrometry method and total reflection X-ray fluorescence 
spectroscopy, an analytical technique whose development and validation for quantifying AuNPs in cells is the focus of this thesis.

\subsubsection{Inductively coupled plasma-based techniques}

Analytical atomic spectrometry refers to a family of analytical techniques which can be used for the detection of trace elements. It has become a field of continuous change. Since the development of arc and high-voltage spark spectrometry for metal analysis in the 1940s, the field evolved to flame emission spectroscopy and flame atomic absorption spectroscopy for solution analysis in the 1950s [49]. The advent of furnace-based atomization in the 1960s made the application of the later even more prevalent. The 1970s were considered the decade of the inductively coupled plasma atomic emission spectroscopy (ICP-AES) where the technique was refined and many commercial instruments were developed [50]. Inductively coupled plasma mass spectrometry (IPC-MS) was developed in the 1980s and along with ICP-AES has since become one of the most commonly used methods for trace analysis because of their low limit of detection (LOD), high sensitivity, precision and analytical throughput [51]. Both techniques have developed into powerful multi-element analytical techniques for trace and ultra-trace analysis of liquid samples directly in solutions or in dissolved solids. This section will review the principles of operation, sample preparation requirements and challenges and discuss technique shortcomings as they pertain to the quantification of NPs in biological tissues.

\subsubsection{Plasma torch components}

A schematic of the components of a typical ICP-MS and ICP-AES system is shown in Figure 1-12. Common to both of these systems is the ICP which is encapsulated in a torch that energizes plasma by inductively heating an inert gas (typically argon) using an electromagnetic coil. From an atomic emission spectroscopy definition, plasma can be considered any gas which contains positive ions and free electrons capable of atomizing, ionizing and exciting the majority of the chemical elements [52]. The plasma is formed in a stream of argon gas which flows through an assembly of concentric quartz tubes at a flow rate ranging from 8 to $20 \mathrm{~L} / \mathrm{min}$ [49]. The argon gas is heated by copper induction coils connected to a radiofrequency (RF) generator which operates at frequencies of 27 or $40 \mathrm{MHz}$ producing a power of $1-2 \mathrm{~kW}$. The argon gas is energized by the current that the magnetic field of the RF coils produces through high-voltage Tesla discharges [53]. The doughnut-shaped plasma (5000-8000 K temperature) remains stable 
and self-sustaining for as long as the magnetic field strength is sufficiently high and the argon gas flows in a symmetrical pattern. The characteristic shape of the plasma is required for efficient sample desolvation, vaporization, atomization, excitation and eventual ionization. This allows the argon gas to excite-ionize a wide range of elements (particularly metals) and enable simultaneous multi-elemental identification [54].

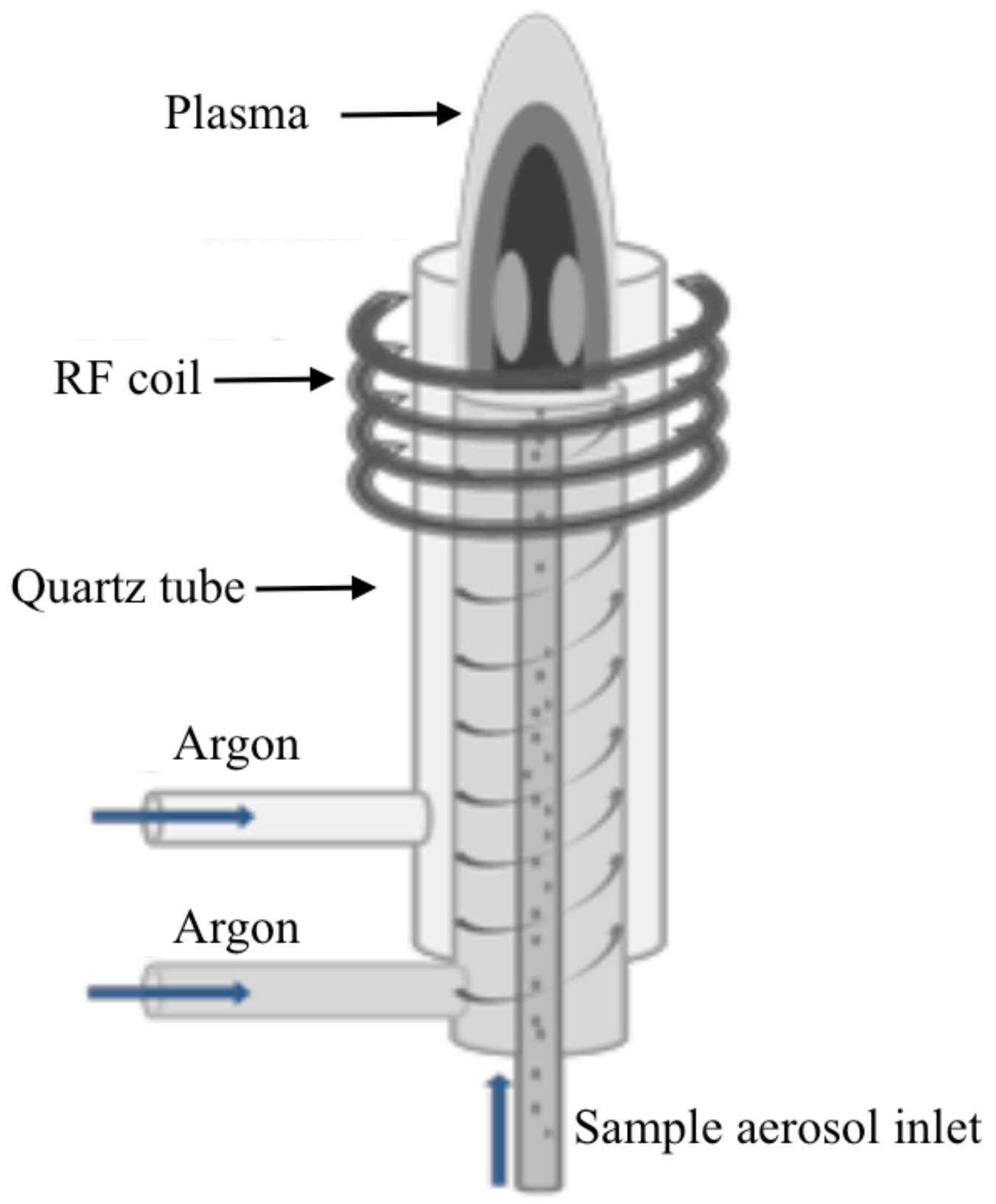

Figure 1-12: Schematics of an ICP plasma torch ( $R F$ = radiofrequency; adapted from [52]). 


\subsubsection{ICP-MS principles of operation}

ICP-MS is used in approximately $80 \%$ of trace analyses being carried out in environmental, geological, semiconductor, biomedical and nuclear application fields [55]. Even though the technique can broadly identify the same set of elements as other atomic spectroscopy techniques, its multi-elemental characteristics, detection limits, isotope capabilities and speed of analysis make the technique highly versatile. Figure 1-13 shows the most common components that make up an ICP-MS system [55]. The liquid sample is typically pumped through a peristaltic pump (1 L/min flow rate) to nebulizer which converts it into a fine aerosol using the argon gas used for the plasma. A spray chamber is then used to separate fine aerosol droplets (1-2\% of the total sample) from the larger ones before transporting them through a sample injector into the plasma torch (as seen in Figure 1-12).

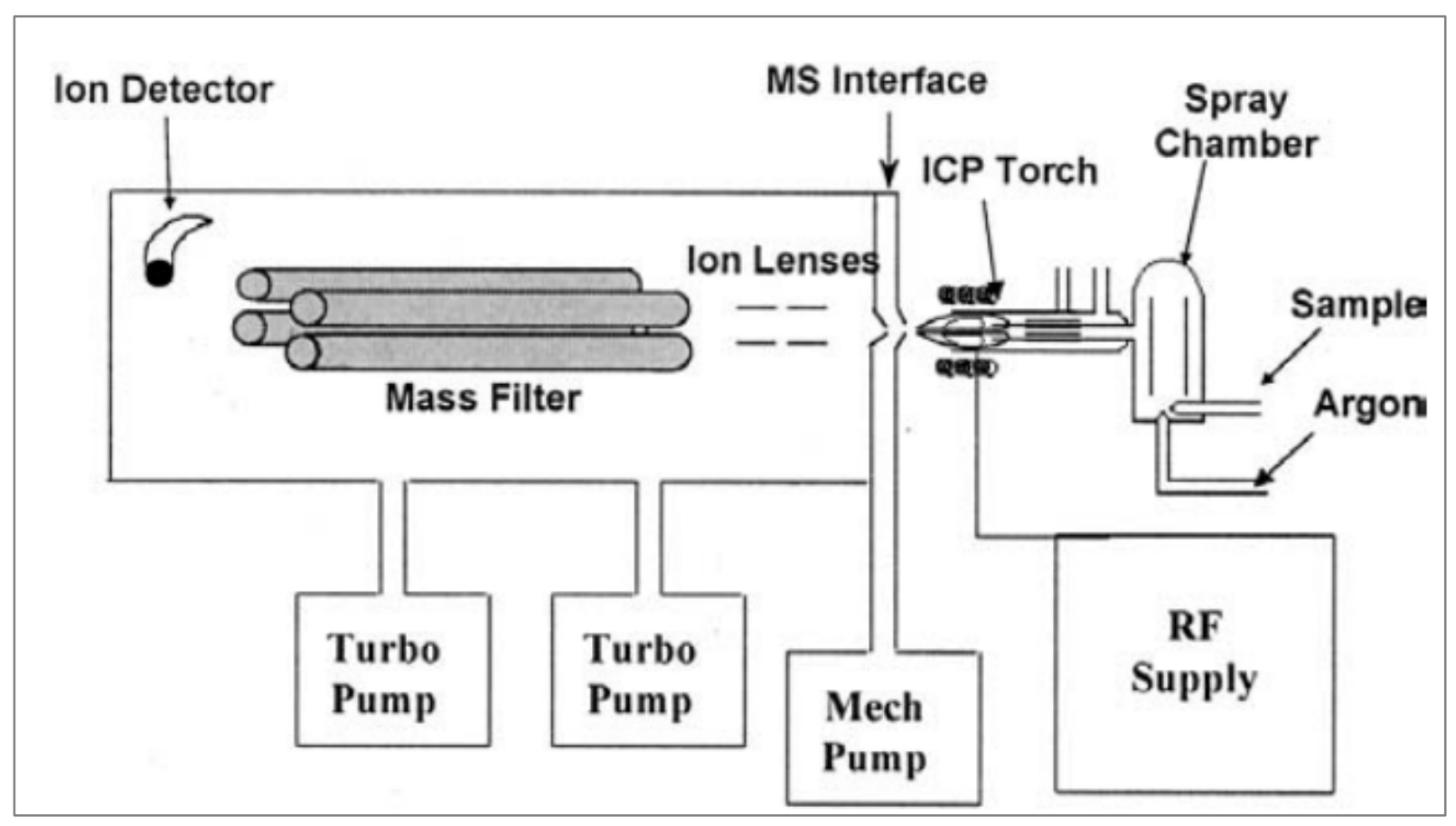

Figure 1-13: Schematic of an ICP-MS setup (MS = mass spectrometer; adapted from [55]).

Unlike in ICP-AES (section 1.4.1.3), the plasma of ICP-MS is positioned horizontally and it generates positively charged ions. Every attempt is made to block the photons from reaching the detector as they introduce unnecessary noise [56]. The production and detection of large quantities of these ions allows ICP-MS to detect with low parts per trillion (ppt) capabilities. Next, these ions are directed to the mass spectrometer through an interface region maintained at 1-2 Torr using a mechanical pump. This is portion of the device is one of the most 
crucial as the ions produced within the plasma (maintained at 760 Torr) must be efficiently guided through the mass spectrometer region kept at approximately $10^{-6}$ Torr. Proper grounding of the RF coil in the torch is paramount in order to avoid the erratic and unpredictable behavior of excess charges. A series of electrostatic lenses called ion optics are required for focusing the ion beam toward the mass separation device while stopping photons, particulates and neutral species from reaching the detector. A turbomolecular pump maintains this region at a vacuum pressure of $10^{-3}$ Torr [57].

The focused ion beam now contains all the analytes as well as the matrix ions before it enters the mass separation device kept at low pressure with a second turbomolecular pump as shown in Figure 1-13. While different types of mass separation devices exist, they all allow analyte ions of a particular mass-to-charge ratio through to the detector while filtering out all the non-analyte, matrix and other interfering ions [55]. This process is either done sequentially or simultaneously. The last step is the conversion of these remaining ions to electrical signals using an ion detector. The ions impinge on a dynode and are converted into electrons which are amplified through sequential multiplication to the adjacent dynode. This electrical signal is then converted into analyte concentration using the ICP-MS calibration standards described in detail in section 4.1.4.

\subsubsection{ICP-AES principles of operation}

As the name implies, atomic emission spectroscopy is based on the emission of radiation from excited atoms and ions and the subsequent measurement of the emitted spectral lines for each element present in the analyte in the form of a spectrum. This differs significantly from ICP-MS where electromagnetic radiation from samples is not measured [52]. Unlike atomic absorption spectroscopy which can only measure one analyte at a time due to the nature of emission/absorption process employed, AES allows for multi-elemental identification. The use of multichannel detectors can measure simultaneously several elements and spectral lines since no particular element-specific radiation source is required. This brings the technique to par with ICP-MS albeit the later has impressive detectability and selectivity.

A schematic of a typical ICP-AES system is shown in Figure 1-14 [58]. In a similar fashion to the ICP-MS setup, the AES systems make use of a plasma torch such as the one shown in Figure 1-12. In both techniques, the torch produces a 10,000 K plasma discharge at the open end of the tube. As a result of the action of the argon plasma particles and the induced 
power from the RF generator, the analyte aerosols are desolvated and dissociated into free atoms and ions.

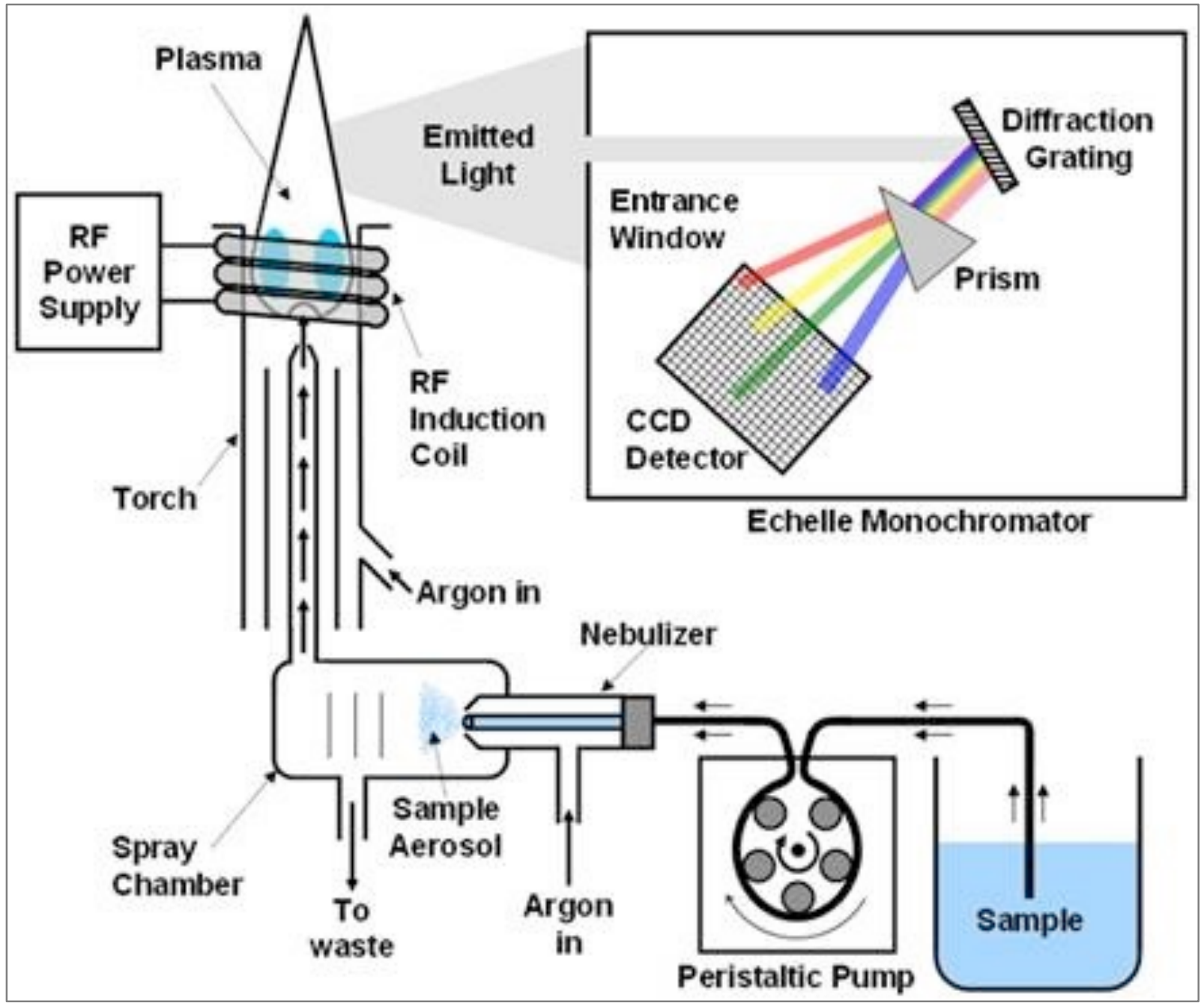

Figure 1-14: Schematic of an ICP-AES setup (adapted from [58]).

However, a fundamental difference exists between the techniques in the next step. Instead of the analyte ions passing through to a mass spectrometer, they become excited and subsequently de-excited from the plasma discharge. The captured energy is released as a spectrum of characteristic atomic and ionic lines [59]. A spectrometer which is equipped with a monochromator or polychromator and a CCD detector quantizes the measured intensity of the emitted photons and produces a spectrum. The latter is converted into analyte concentration using standard calibration curves which have been obtained through the use of standard solutions (described in detail in section 1.4.1.4). Figure 1-15 shows a schematic of the comparison between ICP-MS and ICP-AES. Both techniques are capable of covering almost all elements 
(Figure 1-15a) but ICP-MS outperforms its AES counterpart with smaller detection limits (fg/ml vs. ng/ml) as show in Figure 1-15b. Additionally, ICP-MS is capable of analyzing radioisotopes [53].

(a)

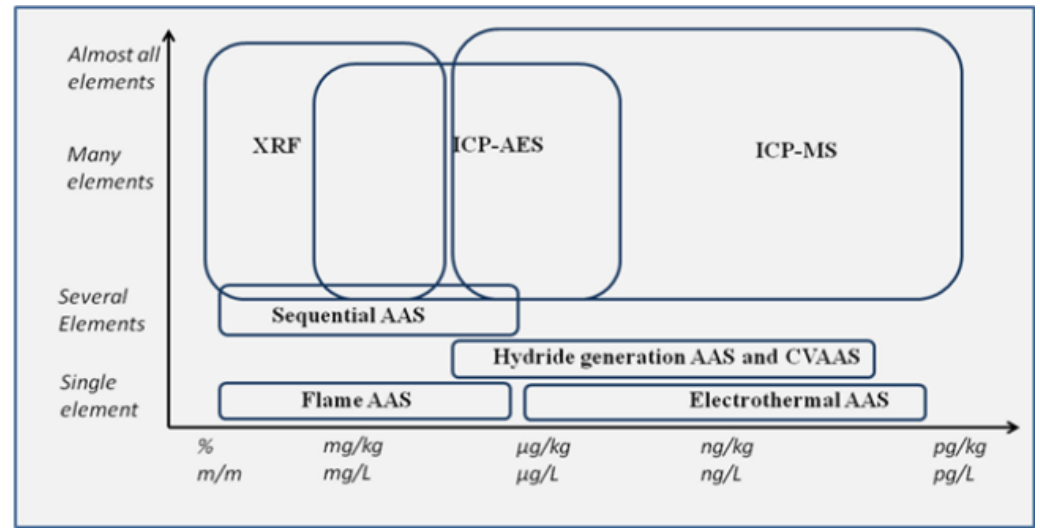

(b)

\begin{tabular}{|c|c|c|}
\hline & ICP-MS & ICP-AES \\
\hline Multi-element capability & + & + \\
\hline $\begin{array}{l}\text { Matrix and minor } \\
\text { elements } \\
\text { (stoichiometry) }\end{array}$ & + & ++ \\
\hline Trace elements & ++ & + \\
\hline Ultratrace elements & ++ & - \\
\hline Limits of detection & $\begin{array}{l}\mathrm{pg} / \mathrm{ml}- \\
\mathrm{fg} / \mathrm{ml}\end{array}$ & $\mathrm{ng} / \mathrm{ml}$ \\
\hline $\begin{array}{l}\text { Sample amount of analyte } \\
\text { Isotope analysis } \\
\text { (isotope dilution analysis) }\end{array}$ & $\begin{array}{l}\text { ng-fg } \\
++\end{array}$ & $\begin{array}{l}\mu g-n g \\
-\end{array}$ \\
\hline Dynamic range & $10^{9}$ & $10^{8}$ \\
\hline
\end{tabular}

Figure 1-15: (a) Approximate illustration of the working range of several atomic spectroscopy techniques and their capability of providing multielemental analysis $(\mathrm{XRF}=\mathrm{X}$-Ray Fluorescence, AAS = Atomic Absorption Spectroscopy, CVAAS = Cold Vapor AAS; adapted from [52]). (b) Features of ICP-MS and ICP-AES for the analysis of liquid samples (adapted from [53]).

\subsubsection{External calibration and sample preparation}

In ICP based techniques, quantitative analysis is achieved by means of external calibration. The calibration curve is prepared from high purity grade stock solution, and the intensities of the calibration standards are plotted as a function of the known analyte concentration [60], [61]. A mathematical relationship between the measured intensity and the calibration standard analyte concentration can be expressed by a linear regression model $y=m x+b$, where $\mathrm{y}$ is the measured response, $\mathrm{x}$ is the analyte concentration and $\mathrm{m}$ and $\mathrm{b}$ are the slope of the line and the $\mathrm{y}$ intercept, respectively. The sample's unknown analyte concentration can then be calculated using the signal obtained from the sample and the calibration curve equation [61].

The efficiency of the nebulizer is affected by matrix of the sample, such as acidity and salt content [62]. Since the calibration standards are made from high purity standard solutions, external calibration does not compensate for this matrix effect [60]. Therefore, the sample matrix must thus be matched to that of the calibration standards. Moreover, as discussed in sections 1.4.1.2 and 1.4.1.3., ICP based techniques require the sample to be introduced to the nebulizer in aqueous form. ICP samples are thus pre-treated to ensure matrix-matching and complete digestion of samples. 
There are several ways to pre-treat ICP samples depending on the analyte of interest and initial sample form. For organic samples, the most popular forms of sample decomposition are wet ashing and microwave digestion [61]. Wet ashing utilizes oxidizing acids, open glass vessels and external heat source to decompose organic sample matrix [63], while microwave digestion is performed with oxidizing acids in a microwave oven. In both cases, the choice of oxidizing acids is dependent on the nature of the matrix and the analyte. For instance, biological samples can only be oxidized by nitric acid, while noble metals can only be dissolved by Aqua regia (a 1:3 vol/vol mixture of nitric acid to hydrochloric acid) [63].

The duration of sample pre-treatment is dependent both on the analyte and mode of decomposition. Generally, for wet ashing, sample is placed in an open glass vessel and the appropriate choice of acid is added to the sample. The mixture is then heated on hot plate until the sample is fully decomposed. The temperature of the decomposition process is dictated by the boiling point of the oxidizing acid and the process can take several hours [61], [63]. In contrast, given the high temperature and pressure of microwave ovens, microwave digestion takes only a few minutes [61]. This speed offered by microwave digestion has made it a popular choice for ICP sample decomposition [61]. That being said, microwave digestion is limited by the number of samples that can be simultaneously digested. Wet ashing typically allows for the heating of up to 24 test tubes while microwave digestion is limited only to 14 samples [63].

ICP sample preparation doesn't have a one-size-fits-all approach. Choice of reagents, duration of sample pre-treatment, mode of digestion is all dependent on the matrix of the sample and the analyte of interest. Therefore, an appropriate method of sample preparation must be carefully chosen for each sample.

\subsubsection{Shortcomings of the ICP-based techniques}

The significance of reliable quantification of NPs in biological tissue becomes clear when highlighting the wide range of biomedical applications summarized in section 1.3. ICP-based quantification methods show a lot of potential in the quantification of NPs given their low LOD and high throughput [51]. However, these methods require that the NPs and the surrounding matrix (intra/extracellular biomolecules) be completely decomposed through acid digestion of the suspension [63]. Additionally, samples must also be matrix matched with the calibration standards to ensure accurate quantification [63]. These sample preparation requirements make ICP based techniques very tedious and long process [64], [65]. Moreover, ICP spectrometers 
require large sample volume $(5-10 \mathrm{ml})$ [60]. This can be problematic when dealing with samples with very small amounts of analyte. Thus, despite their popularity, the heavy influence of sample preparation on quantification accuracy as well as the large volume requirement limit ICP based techniques as it pertains to quantification of nanoparticles in organic matrix.

\subsection{Total reflection X-ray fluorescence spectrometry}

In 1923 Compton discovered the phenomenon of total reflection of X-rays where below a certain angle the reflectivity of a flat target strongly increased [66]. 50 years after Compton's discovery, Yoneda and Horiuchi proposed analysis of small amount of material on flat reflecting target using total reflection x-rays [66], [67]. Since then, total reflection X-ray fluorescence (TXRF) based analysis has become a well- recognized analytical tool with high sensitivity and low detection limit [66]. TXRF based method of analysis has been extensively developed and has become a high performing method comparable to other spectroscopic methods [67]. An evergrowing interest in TXRF has given rise to a wide variety of applications. With several commercially available spectrometers worldwide, the application of TXRF ranges from analysis of water, oil, aerosols to body fluids and tissues [66]. This section will review the physical principles and instrumentation of TXRF, sample preparation and quantification, as well as applications and suitability of nanoparticle quantification.

\subsubsection{Physical principles}

X-ray fluorescence based analysis is a spectrochemical method based on irradiation of sample by a primary beam [67]. The interaction of primary radiation with the sample results in photoelectric effect and, Rayleigh and Compton scattering. In Rayleigh scattering the direction of the primary beam is changed without losing energy. In Compton scattering the interaction of photon with loosely bound outer electron results in deflection of the primary beam and loss of energy where the amount of energy lost is related to the deflection angle [67]. In contrast to Rayleigh and Compton scattering, in photoelectric effect an inner shell electron is ejected upon interaction of the primary beam with the sample, given that the primary beam has energy larger than the binding energy of the shell. The vacancy created by the ejected electron is filled by an upper shell electron. This transition of orbital electrons is accompanied by the emission of X-ray lines known as characteristic X-rays. Each element has a unique electron structure and energy levels, and thus consequently a unique set of spectral lines. This characteristic X-ray fingerprint 
is the foundation of X-ray fluorescence based identification and quantification of elements. The nomenclature of characteristic X-rays is based on initial and final state of the transition [68]. For instance, if an electron is ejected from the K-shell and the vacancy is filled with an electron from the L-shell, the emitted line is known as Ka (Figure 1-16).

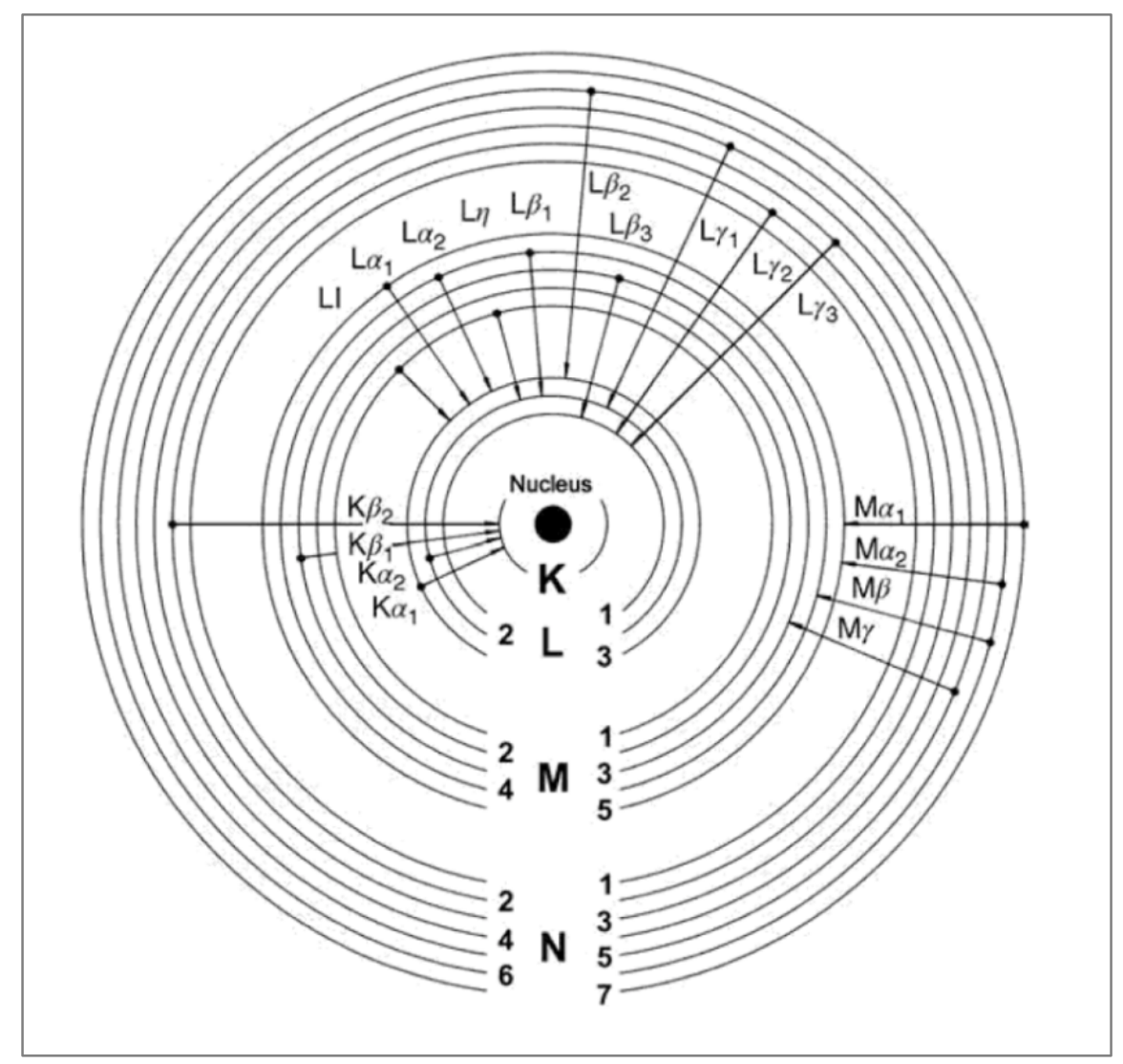

Figure 1-16: Possible electron transitions in a heavy atom. The resulting characteristic X-rays and corresponding nomenclature are shown (adapted from [67]).

Total X-ray fluorescence is a variation of $\mathrm{x}$-ray fluorescence with a special geometry. Unlike commercially available XRF spectrometers where the primary beam and detector are positioned $45^{\circ}$ to the sample, TXRF uses a primary beam at an angle of $0.1^{\circ}$ or less and a detector positioned $90^{\circ}$ to the sample [67]. The low incident angle of the primary radiation allows for the beam to be totally reflected, forcing the radiation to barely penetrate into the reflector, thus drastically reducing the background contribution from Rayleigh and Compton scattering [66]. Additionally, excitation efficiency is increased since the sample is excited both by the primary beam and the reflected beam [66], [67]. The $90^{\circ}$ geometry between the detector and the sample further reduces background contributions from Compton scattering since the 
differential cross-section for Compton scattering becomes a minimum at $90^{\circ}$ [69]. Ultimately the unique geometry of TXRF significantly reduces background and results in high peak-tobackground ratio, consequently increasing the detection limit by several orders of magnitude and allowing for the detection of nanogram amounts of analyte [66], [69].

\subsubsection{Instrumentation overview}

The basic design of TXRF spectrometer includes an excitation source, a reflective sample carrier and an energy dispersive detector as shown in Figure 1-17. The most common excitation source is an X-ray tube with a Mo anode [66]. The primary beam emitted from the X-ray tube is a combination of the characteristic lines of the molybdenum anode and bremsstrahlung background. Therefore, the shape and spectral distribution of the primary beams needs to be adjusted before hitting the sample carrier in order to decrease spectral background. This is achieved by the use of spectral modification devices such as monochromators and cut-off filter [66], [67]. Proper alignment of spectral modification devices is crucial in order to avoid suppression of primary beam or fleeting of high-energy photons to pass the device or suppression of primary beam [66].

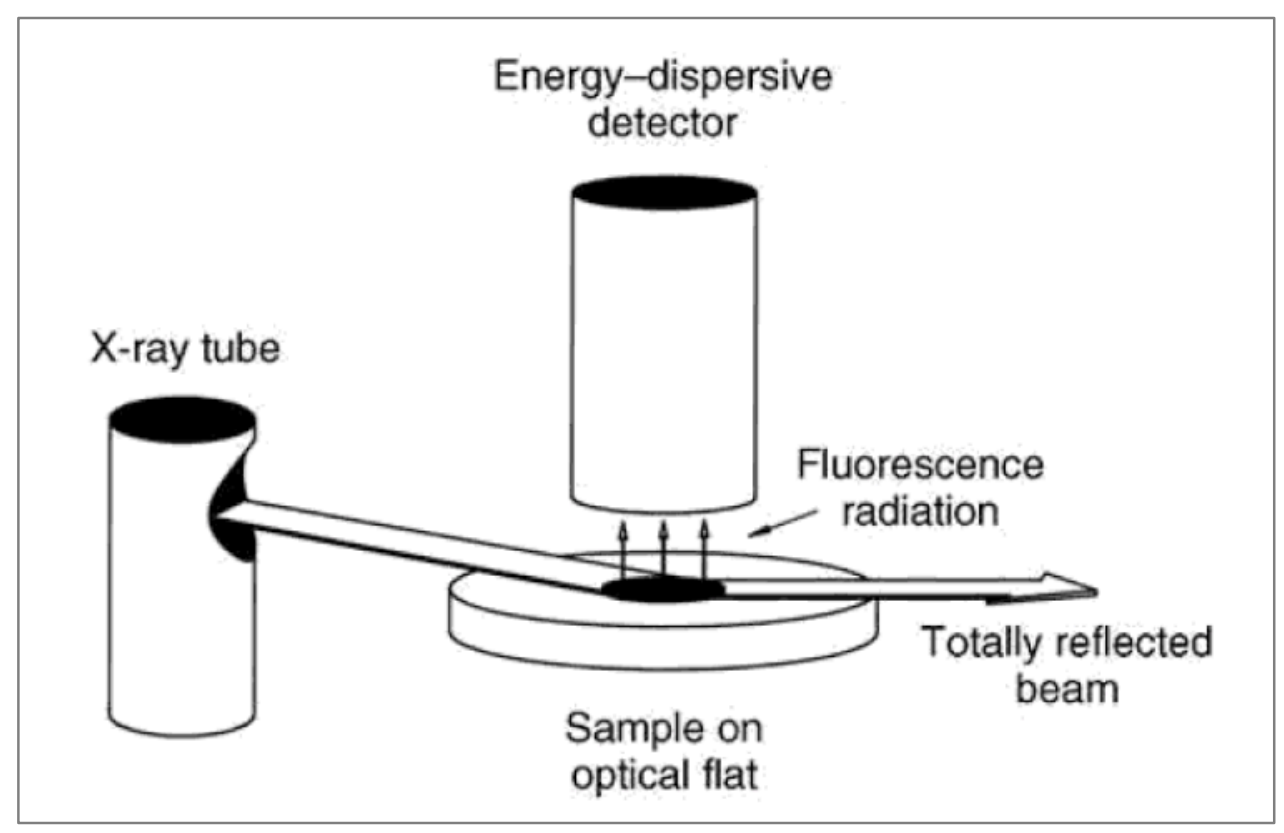

Figure 1-17: Schematic of a TXRF setup (adapted from [67]).

Following the spectral modification devices, the beam travels to the sample carrier. The purpose of the sample carrier is to serve as sample support and provide a highly reflective sample 
surface [70]. As such, there are several requirements a sample carrier must meet. Firstly, a perfectly polished and flat carrier is required in order to meet the conditions of total reflection. A polished carrier can also reduce spectral background as microgrooves in the sample carrier can act as scattering zones [69]. Secondly, sample carriers should be chemically inert in order to avoid reaction with sample. The carriers should also be completely free of contaminants to avoid contamination of sample spectrum. Finally, the sample carrier can't have a fluorescence peak in the spectral region of interest. Several materials have been used as sample carrier, however quartz and Plexiglas remain to be the most commonly used ones [67].

Once the modified primary radiation interacts with the sample, the produced fluorescence radiation needs to be converted to useful data. The X-ray photons emitted from the sample are collected by a detector and voltage pulse proportional to the photon energy is produced for each photon [67]. These pulses are processed and sorted by multichannel analyzer to produce a spectrum. TXRF utilizes Peltier-cooled Silicon Drift detector (SDD). SDD is a small disc consisting of high purity silicon wafer with a thin $\mathrm{P}^{+}$doped cathode front area and drift electrode back area [67]. The incoming X-rays interact with the front area producing several electron-hole pairs in the silicon wafer. The drift rings move the electrons towards the central anode ring and the collected charge is converted into a voltage pulse [67]. Subsequently, the voltage pulse is converted into a readable spectrum.

TXRF instrumentation offers a set up with relatively low maintenance and operating cost. This compact unit is easy to operate and provides a user-friendly process. Moreover, the cost of sample carriers can be kept at minimum since quartz reflectors can be washed and reused. Alternatively, disposable Plexiglas which cost pennies can be used [67]. Some TXRF spectrometers also offer automatic sample changing and recording of spectra, which can further simplify the process.

\subsubsection{Sample preparation}

TXRF is a method that is restricted to very small sample amount, so when large amount of sample is available an appropriate sampling of the specimen needs to be carried out [67]. The sample needs to be thoroughly homogenized prior to setting aside a specimen to ensure the specimen is a proper representative of the sample [67]. The employed method of sample preparation is dependent on the sample that is being analyzed. 
As mentioned in section 1.5.2, a clean reflector free of any impurities is critical for TXRF analysis. The first step in preparing samples for TXRF is thus the cleaning of sample carriers. The procedure used for cleaning sample carriers is outlined below [66], [67]:

1. Sample residue on the carrier is gently removed with lab wipers and acetone.

2. Carriers are heated in detergent bath using special holder designed to avoid scratching of carriers during cleaning.

3. The support with the carriers is cooled down, rinsed with Milli-Q water and transported into another bath.

4. Carriers in their support are boiled in nitric acid bath for 1 to 2 hours.

5. The support with the carriers is cooled down, rinsed with Milli-Q water again and placed in water bath.

6. The carriers are rinsed with Milli-Q water and left to dry.

7. The carriers are covered and put away until they are needed for analysis.

8. On the day of the analysis, a thin hydrophobic film can be added to the sample carriers to avoid running out of samples. This is done by adding a Silicone solution to the carriers and drying them in the oven for 1 hour

9. Finally, a blank spectrum is measured for each reflector to ensure cleanliness.

Various forms of sample preparation exist for TXRF. Samples can be prepared as fine powders, solutions, suspensions or thin sections [67]. Solid bulk samples such as geological samples can be ground down to fine powders. The fine powder can then be mixed with water to form a homogenous suspension. A specimen can be obtained from the resulting solution for TXRF analysis [67]. Environmental samples such as rainwater can be directly analyzed without any pretreatment while mineral samples are digested in open vessel or microwave digestion similar to ICP samples. The combination of acids is chosen based on the sample, however, some acids such as sulfuric acid are not suitable for TXRF because they don't evaporate afterwards [67].

Biological samples, especially those in medical and clinical applications are prepared in a variety of ways. For whole blood sample analysis, microwave digestion by the use of nitric acid is recommended. Plasma and serum samples however can be analyzed directly without any sample pretreatment [67]. Besides blood and plasma, other body fluids such as urine and cerebrospinal fluid can also be analyzed with TXRF. Urine samples do not require any digestion 
or dilution prior to measurements. Cerebrospinal fluid can be decomposed directly in the sample carrier or deposited in the sample carrier and concentrated nitric acid is added to it [67]. Organic tissues can be pretreated with wet ashing and microwave digestion prior to being analyzed similar to ICP methods. However, they can also be measured by directly placing thin sections (5$15 \mu \mathrm{m})$ of the sample on the carrier.

Given the high sensitivity of TXRF, regardless of the sample pretreatment method, only thoroughly cleaned instruments and carriers should be used. All samples must be prepared only with pure water and pure reagents. Additionally, all samples must be prepared under fume hood to avoid any contamination.

\subsubsection{Quantification and limits}

In TXRF, quantification is carried out by means of internal standardization. In internal standardization, a known amount of an element that is initially not present in the sample is added to the sample. This internal standard acts as a reference for the analyte and is used to calculated the unknown concentration of the sample analyte. It's best to add the internal standard at an early stage of the sample so the internal standard better mimics any loss of analyte during sample preparation or inhomogeneous excitation [66]. The steps of internal standardization are shown in Figure 1-18. Briefly, an internal standard is added to a sample and the mixture is thoroughly homogenized. A small volume of the spiked solution is deposited on a clean carrier. The sample is then dried, ready to be measured. 


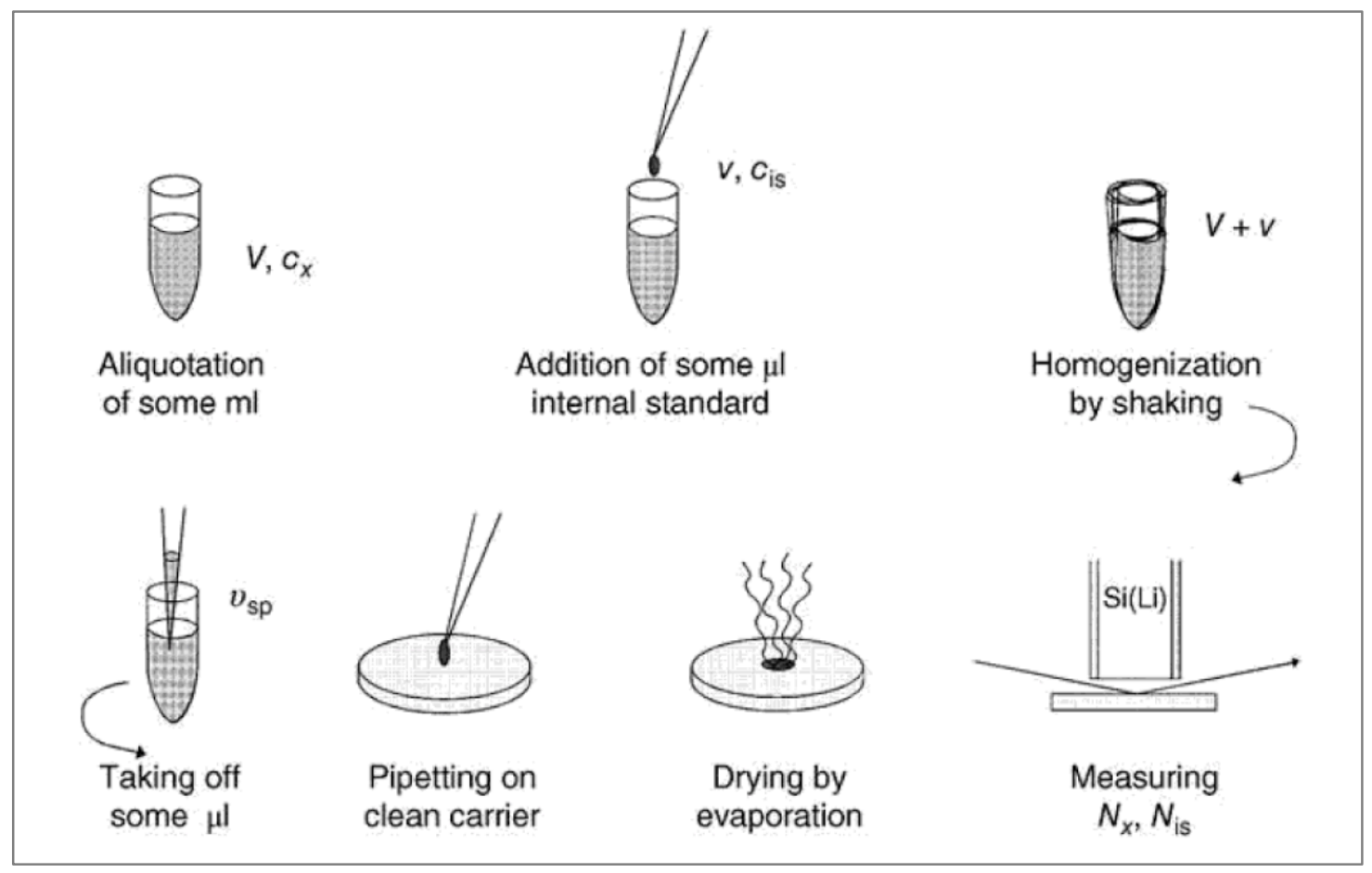

Figure 1-18: Schematic of internal standardization based sample preparation for TXRF analysis. The diagram shows the different steps involved in the process (adapted from [67]).

The unknown concentration of the analyte is then calculated using the following formula,

$$
C_{\mathrm{x}}=\frac{\mathrm{N}_{\mathrm{x}} \mathrm{S}_{\mathrm{is}}}{\mathrm{N}_{\mathrm{is}} \mathrm{S}_{\mathrm{x}}} \mathrm{C}_{\mathrm{is}}
$$

where, $x$ and is are the analyte and internal standard respectively. $\mathrm{N}$ is the net intensity, $\mathrm{C}$ is the concentration and $\mathrm{S}$ is the relative sensitivity. The net intensity of the analyte and the internal standard are determined from the sample spectrum [67]. The sample spectrum contains peaks that have been assigned to specific elements. The net intensity of an element is then the area under the prominent peak of said element [67]. It is obtained by summing all the counts accumulated in the channels representing the peak and subtracting the background (trapezoid area under the peak) [67]. In multi-element analysis, overlap of peaks between different elements is a common issue. In such cases, both the overlapping neighbouring peaks and the background need to be correct in order to obtain the net count. The overlapping peaks can be separated using known elemental intensity ratios or using a library of principal peaks of pure elements [67]. In case of the first method, the 'clean peak' (overlap free peak) is used to determine net intensity of the overlapped peak using the known ratios between their intensities. In case of the later, pure element peaks are superimposed on the sample spectrum. The peak heights are then reduced until 
the pure peaks fit the corresponding peaks from the sample [67]. A variety of spectral analysis software exist to analyze and deconvolve the spectrum and obtain elemental net intensities.

In TXRF, the relationship between intensity of peak, $N_{i}$, and concentration, $C_{i}$, is linear. A proportionality factor, known as sensitivity factor, $\mathrm{S}_{\mathrm{i}}$, can then be described as,

$$
\mathrm{S}_{\mathrm{i}}=\frac{\mathrm{N}_{\mathrm{i}}}{\mathrm{C}_{\mathrm{i}}}
$$

For simplicity, sensitivity factors are reported as relative sensitivities, i.e. ratios of the absolute sensitivities with respect to a specific element. Absolute sensitivities are obtained from calibration line by plotting the measured intensity against the concentration of the element. Since the sensitivity factor is element dependent, this step is repeated for individual elements. The relative sensitivities can then be calculated by,

$$
\mathrm{S}_{\mathrm{i}}=\frac{\mathrm{N}_{\mathrm{i}} / \mathrm{C}_{\mathrm{i}}}{\mathrm{N}_{\mathrm{ref}} / \mathrm{C}_{\mathrm{ref}}} \mathrm{S}_{\mathrm{ref}}
$$

where, $i$ and $r e f$ are the element and the reference element respectively. $\mathrm{S}_{\text {ref }}$ is set to 1 .

Certain conditions have to be fulfilled for the linear relationships between concentration and intensity to hold true. The sample on the carrier is limited in thickness and area. The sample is limited in thickness because TXRF samples need to be 'infinity thin' to be considered free of matrix effect [69]. In infinity thin samples, presence of secondary fluorescence is eliminated and thus emitted intensity is directly proportional to amount of element in the sample. The limiting counting capacity of the detector also poses a limit on the sample area [67]. Additionally, sample must be thoroughly homogenized with the internal standard to ensure accurate quantification.

\subsubsection{Applications}

TXRF based quantification methods offer a multi-elemental analysis with high sensitivity, low detection limit, using small sample volume and relatively easy sample preparation process. These benefits of TXRF have opened up a great variety of applications. In environmental and geological studies, TXRF is being applied to pollution control, ultra-trace water analysis as well as mineral, sediment and soil analysis [66], [67]. In biochemical applications, TXRF is being used for analysis of plants, essential oils, vegetation and wine to name a few [67]. In medical and pharmaceutical applications, TXRF is used for analysis of blood, body fluids and trace elements in cancerous tissues. 


\subsection{Hypothesis, specific aims and thesis organization}

\subsubsection{Hypothesis}

The hypothesis guiding this investigation is that total reflection X-ray fluorescence (TXRF) spectroscopy can quantify the uptake of gold nanoparticles (AuNPs) in breast cancer cell suspensions and provide equivalent recovery rates compared to inductively coupled plasma (ICP) based methods while using a smaller sample volume and less extensive sample preparation.

\subsubsection{Specific aims}

The specific aims (SA) of this thesis are:

SA1: To find the suitable internal standard and fitting model for gold quantification using reference solutions.

SA2: To determine the effects of acid digestion and storage time on gold quantification from suspensions of MDA-MB-231 cells incubated with homemade $10 \mathrm{~nm}$ AuNPs.

SA3: To validate the methods refined in SA1 and SA2 using reference $10 \mathrm{~nm}$ AuNPs solutions incubated with and without cells.

SA4: To compare gold recovery rates obtained with TXRF with ICP-AES on the same incubated cell samples.

\subsubsection{Thesis organization}

This thesis is organized in four chapters. An introduction of AuNPs, their biomedical applications and quantification methods is provided in chapter 1. Overview of the ICP-based techniques, particularly pertaining to the sample preparation is also presented in the same chapter. In chapter 2, the findings for a suitable internal standard and spectra fitting models are summarized using gold and zinc standard solutions. Additionally, the effects of acid digestion and storage time on the quantification of homemade AuNPs incubated with MDA-MB-231 cells are also discussed. This chapter forms the basis of a manuscript submitted to the Journal of Analytical Atomic Spectrometry and some of the results have been presented at the international Denver X-ray Conferences $(2016,2017)$ and the international conference on Total Reflection Xray Fluorescence Analysis and Related methods (2017). Chapter 3 investigates the direct 
comparison of TXRF with ICP-AES on quantifying the uptake of reference and homemade AuNPs in MDA-MB-231 cell suspensions. These results have been presented at the international symposium of trace element in man and animal (2017) and form the basis of a manuscript planned to be submitted to ACS Nano (December 2017). The thesis summary and future directions are presented in chapter 4 . 


\section{TOTAL REFLECTION X-RAY FLUORESCENCE BASED QUANTIFICATION OF GOLD NANOPARTICLES IN CANCER CELLS}

This chapter presents a manuscript that was submitted to the Journal of Analytical Atomic Spectrometry (JAAS).

Manuscript \#: JA-ART-10-2017-000332

Submission date: $03 / 10 / 2017$ 


\title{
Total reflection X-Ray Fluorescence based quantification of gold nanoparticles in cancer cells
}

\author{
Gabriella Mankovskii and Ana Pejović-Milić \\ Department of Physics, Faculty of Science, Ryerson University, 350 Victoria \\ Street, Toronto, Ontario, M5B 2K3, Canada
}

In the last two decades, nanoparticles of different shapes, sizes and materials have been investigated for various nanomedicine applications, ranging from imaging to radiation therapy, in efforts to improve conventional cancer therapies. The current focus of nanomedicine is to increase the delivery and cellular uptake of nanoparticles. Thus, further development and advancement of the field requires accurate means to quantitatively assess nanoparticle concentration in cells. Inductive Coupled Plasma (ICP) based methods are currently being used for nanoparticle quantification. Such methods however require extensive sample preparation and large sample volumes, which poses a challenge when dealing with small sample volume with low concentration. This work describes the development and validation of a total reflection Xray fluorescence (TXRF) based quantification method for trace-level gold nanoparticles in organic matrix. Suitable internal standards, fitting approaches and sample preparation methods that yield acceptable recovery rates were investigated. The developed method was validated with reference material nanoparticles. Recovery rates of $(102.7 \pm 3.7) \%$ and $(100.9 \pm 5.1) \%$ were achieved for nanoparticles in ionic solution and organic matrix respectively. These results suggest that TXRF is an adequate technique to accurately quantify gold nanoparticles uptake in cancer cells. 


\subsection{Introduction}

Since the first description of gold nanoparticle (AuNP) synthesis in 1951 by Turkevich and colleagues [2], nanoparticles have undergone a revolution in terms of their applications in medicine and biology. Although no universal method for the synthesis of AuNPs exists, a wide range of customization features, manipulation of shape, size, surface coating and functionalization enable precise fine tuning of particle properties. One such example is surface plasmon resonance [4], [70] which allows oscillation of gold conduction band electrons in response to external electromagnetic radiation. Another property of AuNPs is their ability to bind to a number of materials and ligands which depend on the solvent used, size of the particle and of course the biomedical application of interest [7]. Moreover, NP bioconjugation has been achieved with a wide range of molecules such as antibodies [8], sugars, lipids, vitamins, peptides and proteins [9] as well as DNA and RNA [10].

It is precisely the versatility of the aforementioned bioconjugation strategies which makes AuNPs attractive for a wide array of biomedical applications. AuNPs have found broad applications in imaging and diagnosis, therapy and more recently in theranostic domains. In dark field microscopy and photoluminescence AuNP-antibody conjugations have allowed for quantitative assessments in binding of non-cancerous and malignant cells [4]. Diagnostic assays using generic lateral flow assays have been designed to detect the presence or absence of a target analyte (ex. mercury in urine [15] or tumor specific antigens [16]) using conjugation of antibodies with AuNPs. In medical imaging, AuNPs have been used as photoacoustic contrast agents owning in part due to their surface plasmon resonance properties and molecular tagging capabilities in tumors [18]. Photodynamic therapy uses AuNPs as carriers of the exogenous photosensitizers [22] while photothermal therapy relies exclusively in the conversion of light into heat from optical absorption of the gold [24]. The high X-ray absorption coefficient of gold has enabled AuNPs to be used as radiation dose enhancers increasing the radiosensitization of both in-vitro cellular cultures [34] and in-vivo tumors [71]. Theranostic agents composed of AuNPs have been recently proposed as a vehicle that will simultaneously deliver imaging (ex. computed tomography) and therapeutic agents (ex. photothermal therapy) for combined diagnosis and therapy [29]. 
The uptake and retention of AuNPs in biological tissues is directly influenced by the choice of the ligand. Its manipulation is necessary for controlling with a high degree of accuracy the accumulation, biodistribution and time kinetics of the NPs inside the cell [35]. The in-vitro achievements in internalization of AuPs present only half of the monumental challenge of delivering particles into the tissue of interest under in-vivo conditions [37], [41]. It is widely reported that the limited, in-vivo therapeutic efficacy of many nanoparticle formulations is attributed to the complexities of the tumor vasculature. Typically, it is estimated that only $0.7 \%$ of systemically administered nanoparticles accumulate within the tumor and an even smaller number reach the inside of tumor cells [42]-[46]. As NP formulation novelties progress to increase cellular uptake, cost-effective and efficient means of assessing the NP delivery in tissue are most certainly warranted.

Cellular quantification of AuNP uptake is mostly reliant on inductively coupled plasma (ICP) based techniques [72]-[74]. These analytical methods have shown a lot of potential given their low limits of detection and high throughput. However, these methods require that the NPs and their surrounding organic matrix be completely dissolved through acidification of the suspension, which is not a trivial task [73]. Fabricius and colleagues reported the significance of the sample preparation on the recovery rate [74] while Allabashi et al studied the influence of the sample matrix on accuracy and precision [72]. These studies and others suggest that the recovery rates of ICP-based techniques are heavily influenced by the sample preparation.

This paper investigates the use of total X-ray fluorescence spectroscopy (TXRF) for the quantification of AuNP internalization within breast cancer cells. TXRF is an analytical technique used for trace element analysis and fundamentally based on Compton's discovery of total reflection from X-ray beams in 1922. Yoneda and Horiuchi developed the TXRF method for microanalysis of samples in 1971 by directing a primary X-ray beam onto a polished quartz carrier with a glancing incident angle smaller than the critical angle for total reflection [75]. Since then, the technique has become relatively accessible and has found wide applications in trace element analysis in the environment, medicine/biology/pharmacology, geology/mineralogy and fine arts/archeology/forensic fields [76]. TXRF is a multielemental quantitative technique with high signal-to-noise ratio requiring only $10 \mathrm{~nm}$ of analytical depth information while yielding high reproducibility (1-2\%) of results [77]. One of its biggest advantages is its requirement for small quantity of analyte $(5-10 \mu \mathrm{l})$, a significant improvement over the ICP 
requirements of 5-10 $\mathrm{ml}$. Moreover, the use of internal standards for quantification is significantly simplified and no matrix effects are present in the technique. The technique also has versatility in being able to measure various physical forms of analytes ranging from residues and powders, surfaces and surface layers, thin films and multilayered structures [78].

This paper will introduce a novel application of TXRF in quantifying the presence of AuNPs within cancer cells. We demonstrate experimentally that a suitable internal standard and fitting software can yield analytically acceptable recovery rates with minimal sample preparation requirements.

\subsection{Materials and methods}

\subsubsection{Chemicals and reagents}

Gold (1000 mg/L), Yttrium (1000 mg/L) and Zinc (10000 mg/L) certified reference solutions were purchased from Ultra scientific (North Kingstown, RI, USA). HPLC grade Hexane solution and Lanthanum certified reference solution $(1000 \mathrm{mg} / \mathrm{L})$ were obtained from VWR international (Radnor, PA, USA). Acids used for Aqua Regia, 70\% Nitric acid and 37\% Hydrochloric acid, were purchased from EDM (Billerica, MA, USA) and Sigma-Aldrich (MO, USA) respectively. The siliconizing reagent Sigmacote ${ }^{\circledR}$ was obtained from Sigma-Aldrich (St. Louis, MO, USA). Two types of AuNPs were used, reference and in-house produced. The $10 \mathrm{~nm}$ reference material AuNPs (NIST RM 8011) were purchased from National Institute of Standards and Technology (Gaithersburg, MD, USA) and the $10 \mathrm{~nm}$ in-house produced AuNPs were synthesized using the Turkevich method [1]. All samples were prepared using ultrapure water $(18.2 \mathrm{M} \Omega \cdot \mathrm{cm})$ from Millipore’s Milli-Q integral water purification system (Billirica, MA, USA).

\subsubsection{Cell culture}

This study used breast cancer cell line. MDA-MB-231 cells purchased from ATCC (Manassas, VA, USA) were cultured in Dulbecco's Modified Eagle Medium (DMEM) with 10\% Fetal Bovine Serum (FBS) at $37^{\circ} \mathrm{C}$ with $5 \% \mathrm{CO}_{2}$. The cells were grown to $80 \%$ confluency then washed with Phosphate buffered Saline (PBS) three times. Following the PBS wash, cells were dissociated from culture dish with $0.25 \%$ trypsin-EDTA. 
For sample preparation and storage analysis, the cells were incubated with in-house synthesized $10 \mathrm{~nm}$ AuNPs for 16 hours prior to PBS wash and trypsinization.

\subsubsection{Sample preparation and measurement}

Freshly cleaned quartz reflectors were siliconized by depositing $10 \mu$ silicone solution (1:500 $\mathrm{v} / \mathrm{v}$ Sigmacote ${ }^{\circledR}$ to $\mathrm{n}-\mathrm{Hexane}$ ), and blank measurements of the reflectors were then taken to ensure reflectors were free of any contaminants.

Gold and Zinc aqueous solutions of varying concentrations, as well as Lanthanum and Yttrium internal standard solutions, were all prepared by diluting the certified reference solutions with appropriate volume of ultrapure water.

In-house synthesized AuNPs and MDA-MB-231 cells incubated with in-house synthesized AuNPs were prepared in two ways: (a) diluted with ultrapure water and (b) treated with Aqua Regia (3:1 v/v hydrochloric acid to nitric acid). These samples were then stored in the fridge for 48 hours.

For recovery rate analysis, reference material AuNPs were treated with: (a) ultrapure water, (b) 3:1 v/v hydrochloric acid to nitric acid and (c) 4:1 v/v hydrochloric acid to nitric acid. The MDA-MB-231 cells mixed with reference material AuNPs were treated with 4:1 v/v hydrochloric acid to nitric acid.

Internal standard was added to each sample and the mixture was vortexed to ensure homogenous sample. Then $5 \mu \mathrm{l}$ aliquot of the solution was deposited into the siliconized reflector and dried in the oven (Boekel Scientific, Festerville, PA, USA) at $80^{\circ}$. Each reflector was rotated by $120^{\circ}$ and $240^{\circ}$ between measurements to assess precision, thus triplicate measurements of all samples were collected. Reflectors were measured for 600 secs per rotation.

\subsubsection{Total reflection X-ray fluorescence spectrometer}

This study was conducted with benchtop S2 PicoFox TXRF spectrometer from Bruker-AXS (Billirica, MA, USA) equipped with, a molybdenum anode X-ray tube operating at $50 \mathrm{kV}$ and $600 \mu \mathrm{A}$ with a primary beam incidence angle of $0.1^{\circ}$. Radiation detection was achieved with a XFlash $\odot$ silicon drift detector with energy resolution of $<150 \mathrm{eV}\left(\mathrm{Mn} \mathrm{K}_{\alpha}\right)$ and $30 \mathrm{~mm}^{2}$ active 
area. Quality assurance tests of the system were routinely performed to ensure optimal operating condition.

\subsubsection{Spectral processing, calculations and statistical methods}

The spectrometer's software package, SPECTRA version 7.5.3.0 (Bruker Nano GmbH, Berlin, Germany), was used for all data acquisition. The acquired spectra were deconvolved and integrated with three different data analysis software - SPECTRA, PyMCA (European Synchrotron Radiation Facility, Grenoble, France) and OriginPro (OriginLab corporation, Northampton, MA, USA). Additionally, Bruker's SPECTRA software offers three different fitting approaches - Bayes, Normal fit and Optimized fit. While details on the SPECTRA software are not available for proprietary reasons, details on PyMCA can be found in literature [79]. In OriginPro, peaks were integrated using a Gaussian function with linear background, and deconvolution of overlapped peaks was achieved by fitting multiple Gaussian peaks using elemental relative intensities.

Integration and deconvolution of the X-ray fluorescence peaks results in net count of each peak. The concentration of the analyte is then calculated with the following formula,

$$
C_{i}=\frac{N_{i} S_{i s}}{N_{i s} S_{i}} C_{i s}
$$

where $\mathrm{N}_{i}$ and $\mathrm{C}_{\mathrm{i}}$ are the net count and concentration of the analyte and $\mathrm{N}_{\text {is }}$ and $\mathrm{C}_{\mathrm{is}}$ are the net count and concentration of internal standard. The relative sensitivities of the analyte and internal standard are represented by $\mathrm{S}_{\mathrm{i}}$ and $\mathrm{S}_{\text {is }}$ respectively.

Precision tests were performed using the relative standard deviation (RSD\%),

$$
\mathrm{RSD} \%=\frac{\sqrt{\frac{1}{\mathrm{~N}} \sum_{\mathrm{i}=1}^{\mathrm{N}}(\mathrm{x}-\overline{\mathrm{x}})^{2}}}{\overline{\mathrm{x}}} * 100
$$

The limit of detection of the analyte was determined by [32],

$$
\mathrm{LOD}=\frac{3 \mathrm{C}_{\mathrm{i}} \sqrt{\mathrm{N}_{\mathrm{b}}}}{\mathrm{N}_{\mathrm{i}}}
$$

where $\mathrm{N}_{\mathrm{b}}$ is the background net count of the analyte peak.

The effect of sample storage time was assessed using one-way ANOVA, while the effect of the two types of sample preparation methods were compared by t-test. The effect was considered statistically significant if $\mathrm{p}<.05$. 


\subsection{Results and Discussion}

\subsubsection{Determination of Suitable Internal Standard}

Quantitative TXRF analysis is carried out by internal standardization, where a known amount of an element that is similar to the analyte but isn't present in the sample is added to the sample in order to calculate the unknown amount of analyte in the sample [67] as shown by equation 2.1. Consequently, finding a suitable internal standard is a crucial step. Considering the rich elemental composition of cellular suspensions, Yttrium was chosen as an internal standard. However, as shown in Figure 2-1, the resulting recovery rates were low irrespective of the fitting approach used. In order to improve the recovery rate, as per the recommendation of the vendor, the sensitivity factor of gold was recalculated using instructions obtained directly from the vendor. The new sensitivity factor of gold resulted in analytically acceptable recovery rates (i.e. $90-110 \%$ [80]) for the Optimized and Normal fit methods, while the recovery rates of the other fitting methods remained low.

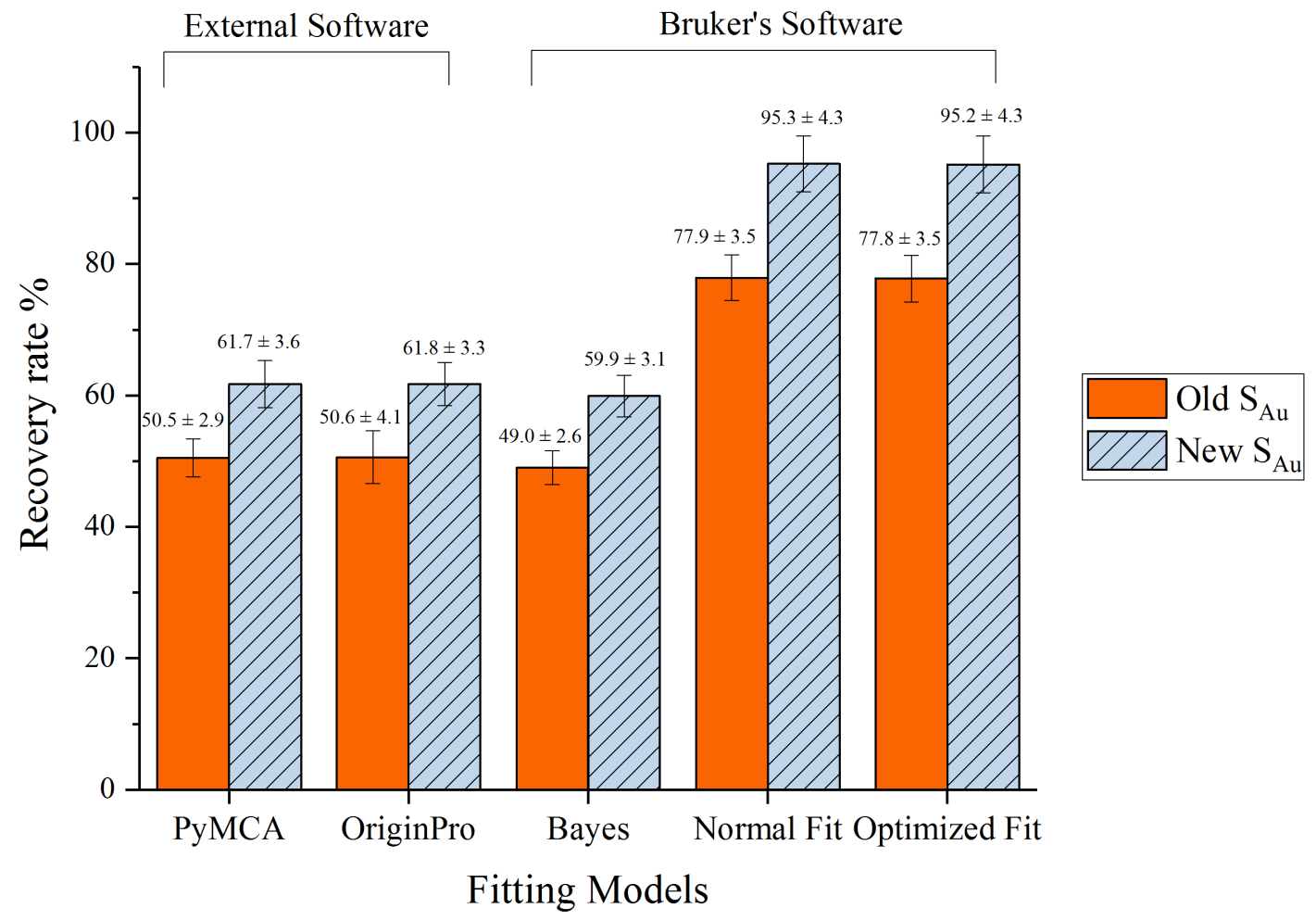

Figure 2-1:Comparison of PyMCA, OriginPro and Bruker's fitting software. Results show recovery rate of $1 \mathrm{ppm}$ $\mathrm{Au}$ certified reference solution with $1 \mathrm{ppm} \mathrm{Y}$ as an internal standard using the original and newly calculated sensitivity factor of $\mathrm{Au}$. The error bars represent the standard deviation of recovery rates from using 3 separate reflectors with 3 rotations per reflector. 
The higher $(\sim 30 \%)$ recovery rate for the Normal and Optimized fits (Figure 2-1) compared to the PyMCA and Origin can be explained by examining the fitting approaches. In this work, we attempt to quantify Au concentrations as low as parts per billion levels. As the analyte concentration decreases, the peak intensity also decreases [66], [77] making it challenging to fit non-prominent, lower count peaks. Thus, in PyMCA and Origin, the most prominent peak of both the internal standard and analyte was used to obtain the net count and calculate concentration of the analyte as shown by equation 2.1, while the rest of the analyte and internal standard peaks were not taken into account. In Bruker's Normal and Optimized fits, one can speculate that peaks other than the prominent may be taken into account when computing the net count.

Given the proprietary nature of Bruker's SPECTRA software, the use of external fitting software such as PyMCA and OriginPro is preferred since their transparency allows control and configuration of fitting parameters. As such, other elements were investigated as potential internal standards in efforts to improve the recovery rate of the external software. Considering that $\mathrm{La}$ is not present in cell cultures and doesn't suffer from spectral interference with elements present in cell suspensions, it was investigated as an internal standard. Additionally, La is the same series as $\mathrm{Au}$, thus mimicking $\mathrm{Au}$ more closely. As shown in Figure 2-2, with La as the internal standard, the recovery rates of Au with the external software improved to analytically acceptable ranges of $102.3 \%$ and $97.6 \%$. Interestingly, Optimized and Normal fit performed worse while Bayes performed better with the L-series internal standard. 


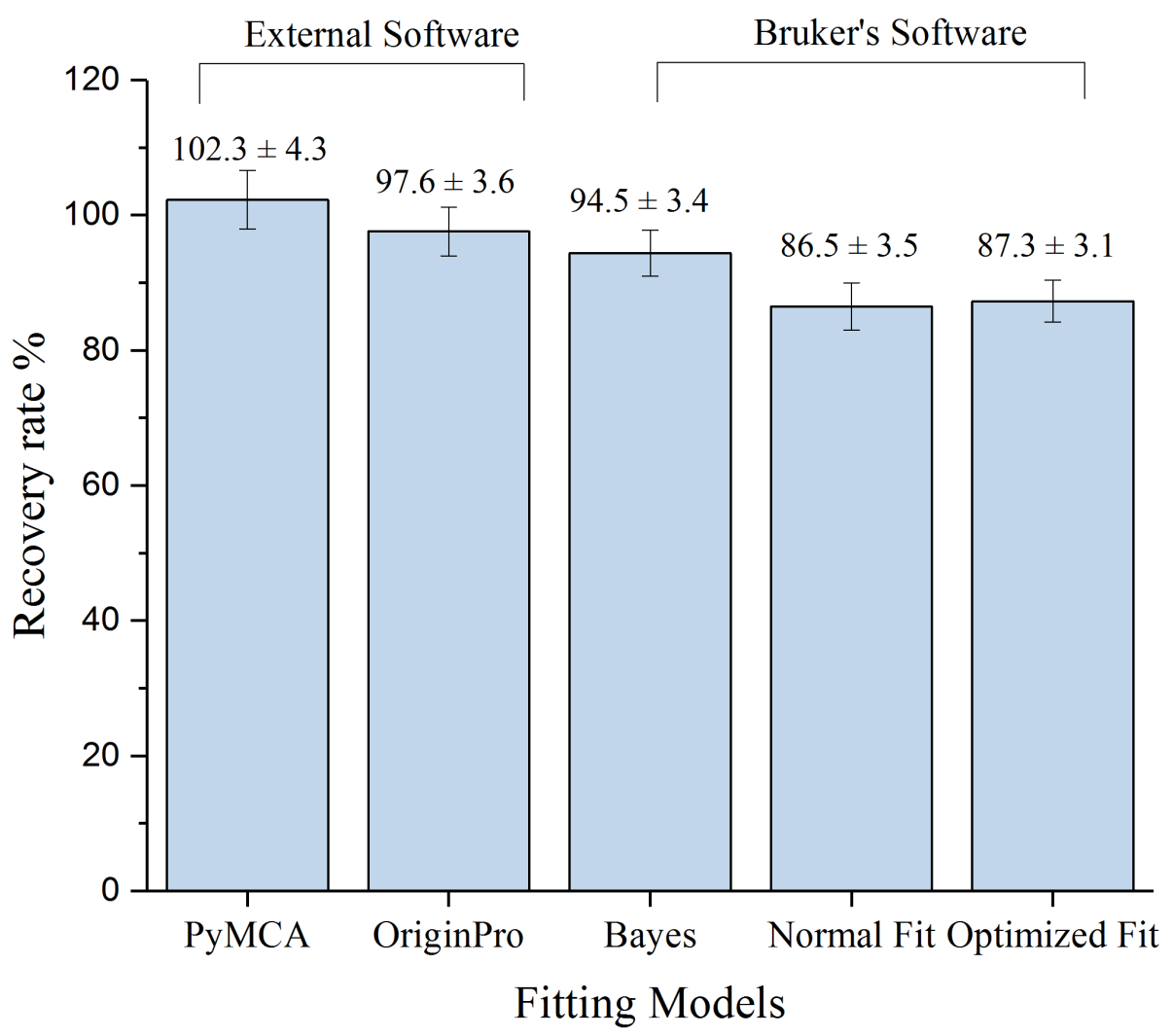

Figure 2-2: Comparison of PyMCA, OriginPro and Bruker's fitting software. Results show recovery rate of 1 ppm Au certified reference solution with $1 \mathrm{ppm}$ La as an internal standard using the newly calculated sensitivity factor of $\mathrm{Au}$. The error bars represent the standard deviation of recovery rates from using 3 separate reflectors with 3 rotations per reflector.

Furthermore, various concentrations of gold reference solution (10 ppb - $1 \mathrm{ppm})$ were investigated against $\mathrm{La}$ internal standard to evaluate dependence of the recovery rate on the concentration of analyte. The lower limit (10 ppb) was chosen as it was calculated to be the LOD in this case. All samples were measured for 600 secs with the exception of the 10 ppb samples which were measured for 1800 secs. Since the detection limit is inversely proportional to measurement time [81] it was necessary to increase measurement time in order to detect the peak of $10 \mathrm{ppb}$ gold. In all cases, acceptable recovery rates were achieved, and it was concluded that La was a suitable internal standard for the quantifying Au. The obtained results are shown in Figure 2-3. 


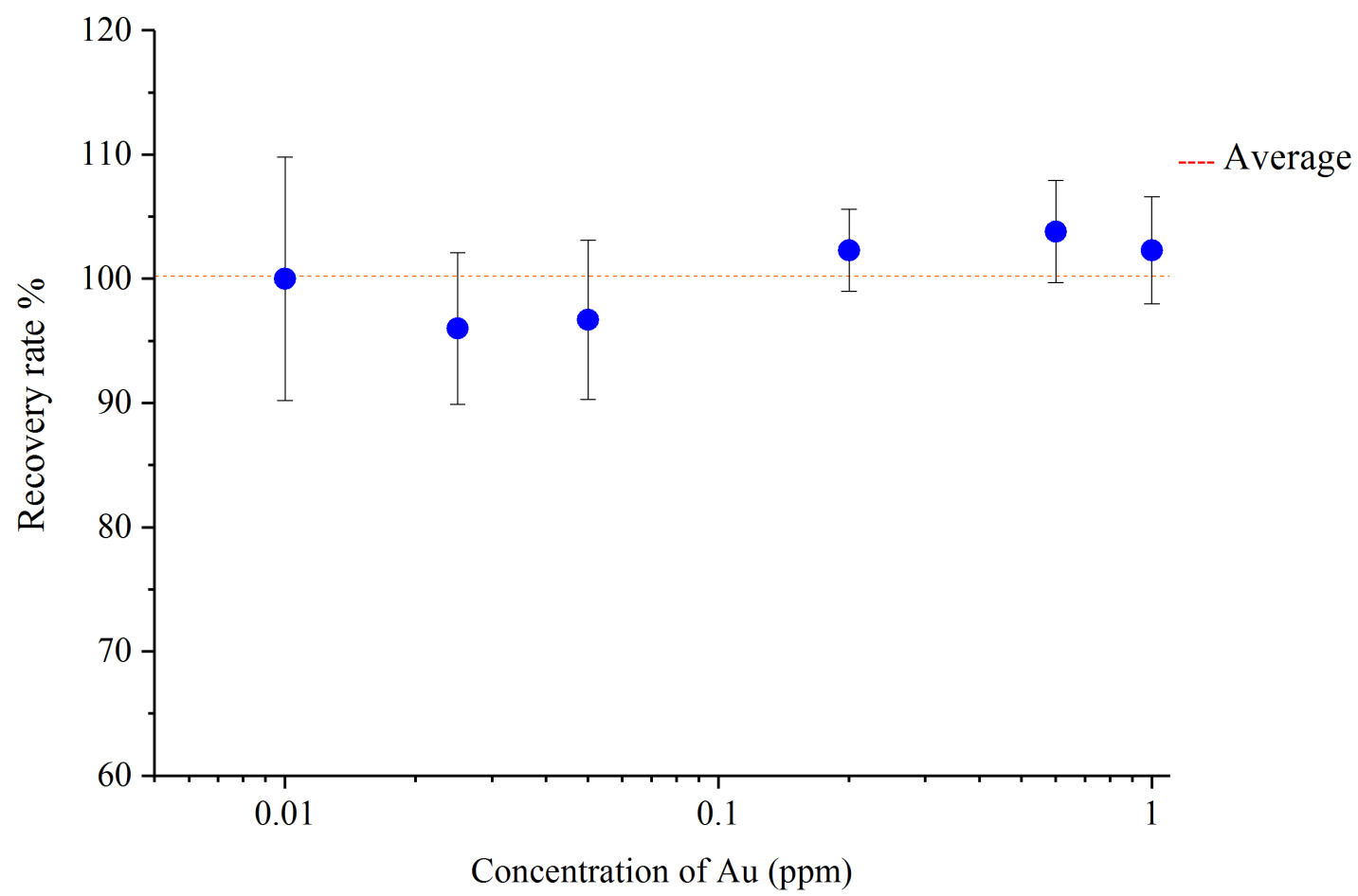

Figure 2-3: Evaluation of dependence of recovery rate on analyte concentration. Results show the recovery rate of $0.01 \mathrm{ppm}, 0.025 \mathrm{ppm}, 0.05 \mathrm{ppm}, 0.2 \mathrm{ppm}, 0.6 \mathrm{ppm}$ and $1 \mathrm{ppm}$ Au certified reference solutions with $1 \mathrm{ppm}$ La as an internal standard using the newly calculated sensitivity factor of $\mathrm{Au}$. The error bars represent the standard deviation of recovery rates from using 3 separate reflectors with 3 rotations per reflector. Data was analyzed with PyMCA

\subsubsection{Effect of Zinc on Gold recovery rate}

Spectral interference is a common problem in TXRF based analysis, especially in multielemental samples [67]. In this case, the $\mathrm{Zn} \mathrm{K}_{\beta}$ peak $(9.57 \mathrm{keV})$, naturally present element in this cell culture, overlaps with the $\mathrm{Au} \mathrm{L}_{\alpha}$ peak $(9.71 \mathrm{keV})$ as shown in Figure 2-4. 


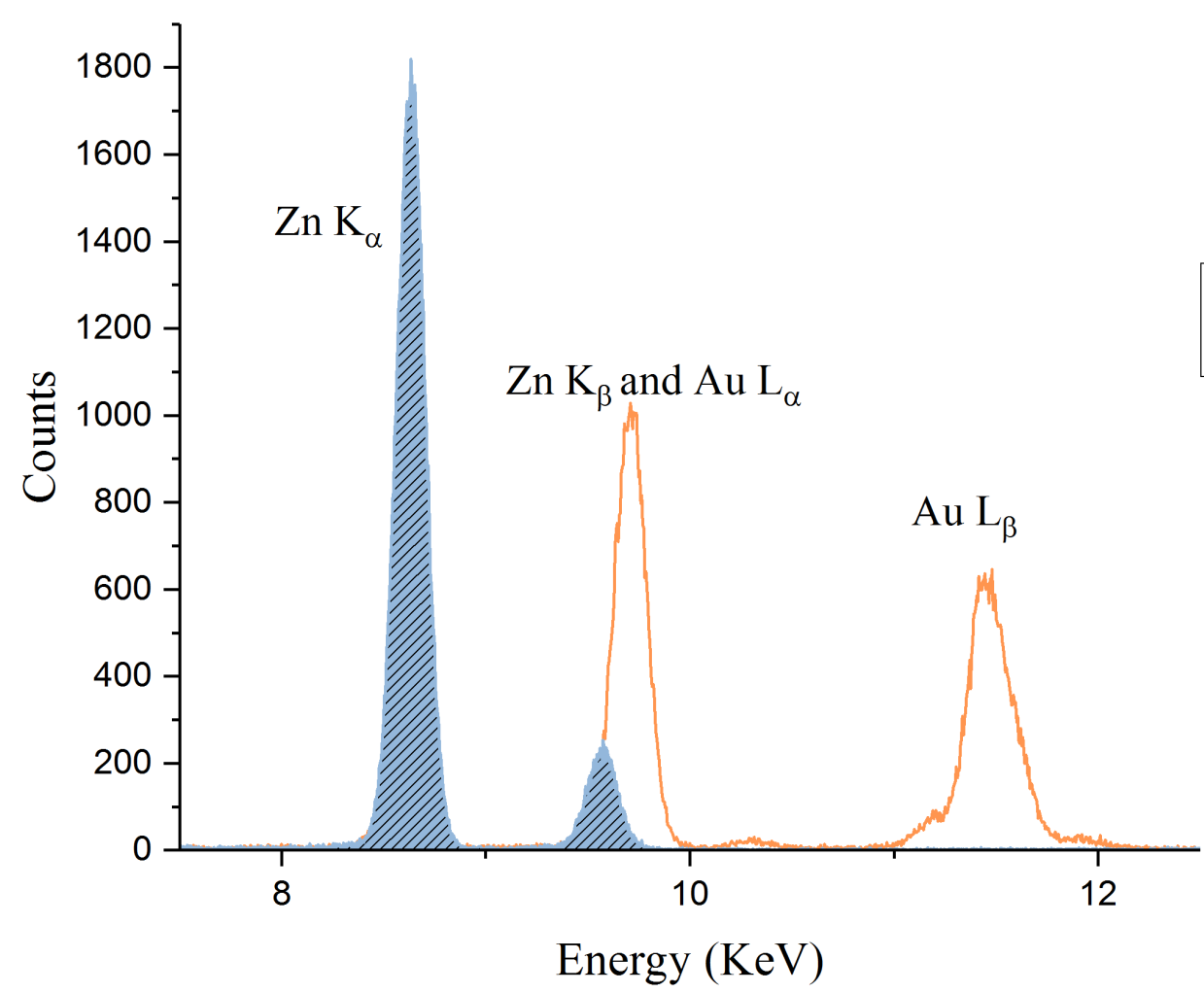

Figure 2-4: Representative TXRF spectrum of $1 \mathrm{ppm} \mathrm{Zn}$ and $1 \mathrm{ppm}$ Au certified reference solution. The spectrum shows spectral overlap of $\mathrm{Zn} \mathrm{K}_{\beta}$ and $\mathrm{Au} \mathrm{L}_{\alpha}$ peak.

The goal of this experiment was to investigate the deconvolution efficacy of the three fitting software. Samples of $1 \mathrm{ppm}$ certified reference Au solution with Zn concentrations of 0.5, 1 and $2 \mathrm{ppm}$ were made to assess the three fitting software and the effect of spectral overlap on the recovery rate of Au. Quantification was carried out with $\mathrm{La}$ as the internal standard as established in section 2.3.1. The obtained recovery rates of Au for each software are shown in Figure 2-5. 


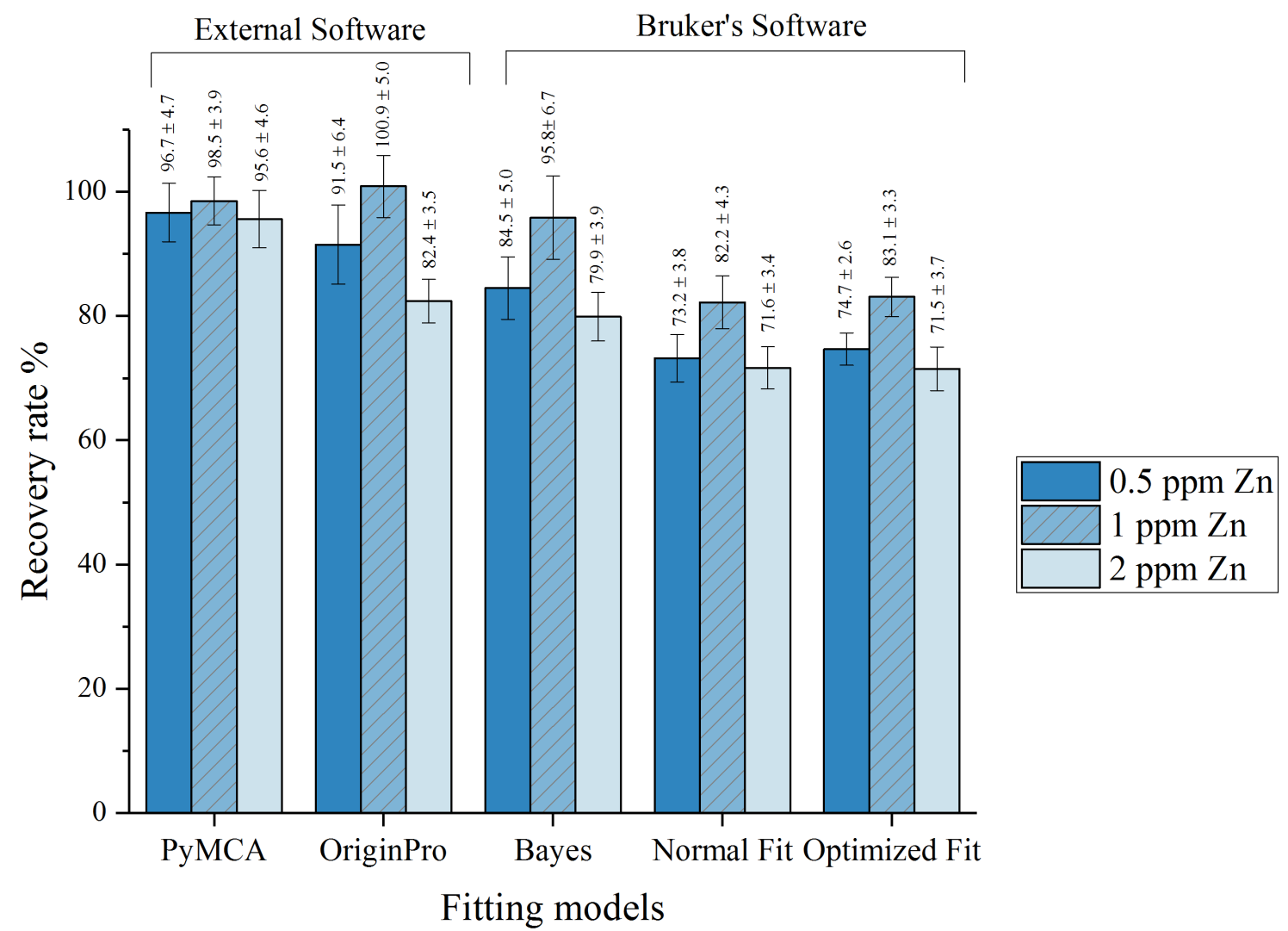

Figure 2-5: Investigation of deconvolution efficacy of PyMCA, OriginPro and Bruker's fitting software with varying concentrations of $\mathrm{Zn}$. Results show the recovery rate of $1 \mathrm{ppm}$ Au certified reference solution with $0.5,1$ and $2 \mathrm{ppm}$ $\mathrm{Zn}$ and $1 \mathrm{ppm} \mathrm{La}$ as an internal standard using the newly calculated sensitivity factor of Au. The error bars represent the standard deviation of recovery rates from using 3 separate reflectors with 3 rotations per reflector.

The results show that Normal and Optimized Fit achieved the lowest recovery rates for all concentrations of $\mathrm{Zn}$. This was expected since they both gave the lowest recovery of Au with La as the internal standard (Figure 2-2). Both Bayes and OriginPro fitting routines performed well when the concentration of $\mathrm{Zn}$ was the same as the concentration of $\mathrm{Au}$ present in the sample. However, they both gave lower recovery rate when the concentration of $\mathrm{Zn}$ varied compared to the concentration of Au. These results suggest that the success of the spectral deconvolution for OriginPro and Bayes is dependent on the amount of $\mathrm{Zn}$ present relative to the amount of $\mathrm{Au}$. This could potentially be an issue for cell cultures rich in $\mathrm{Zn}$ that are incubated with very low concentrations of gold or cell cultures with very low AuNPs uptake.

Unlike Bruker's SPECTRA and OriginPro fitting approaches, PyMCA based approach was able to achieve high recovery rates regardless of the amount of $\mathrm{Zn}$ added. The success of PyMCA based approach over the OriginPro based fitting could be accounted by the sophistication of the software. The OriginPro based approach relied on simple Gaussian model to 
deconvolve the peaks while PyMCA offers and allows the control of several fitting parameters such as tailing and anchors to get more accurate results [82]. OriginPro can also implement similar functionalities. However, the process has to be added manually and repeated for individual peaks, making it a time-consuming process.

Given the results, $\mathrm{La}$ as the internal standard accompanied with PyMCA spectrum fitting is recommended to achieve acceptable peak deconvolution and high recovery rates for the quantification of $\mathrm{Au}$ in cell cultures.

\subsubsection{Sample preparation and storage analysis}

Fundamental principles of TXRF dictate that the sample must be homogenous and free of matrix effect [67]. Thus, a suitable sample preparation process needs to be determined for cell cultures incubated with AuNPs. Samples diluted in ultrapure water and digested in Aqua Regia were investigated to determine if acid digestion is necessary to meet such requirements. The samples were also measured over 48 hours long period to investigate the effect of sample storage time on the accuracy of the quantification. As the goal of this experiment was to determine an appropriate sample preparation method, the absolute values were not of interest for this specific experiment, rather the focus was on the relative values obtained from the two methods of sample preparation and the gold concentration change, if any, over the $48 \mathrm{hrs}$. Figure 2-6 shows the results for in-house synthesized $10 \mathrm{~nm}$ AuNPs and MDA-MB-231 cells incubated with in-house synthesized $10 \mathrm{~nm}$ AuNPs. 
(A)

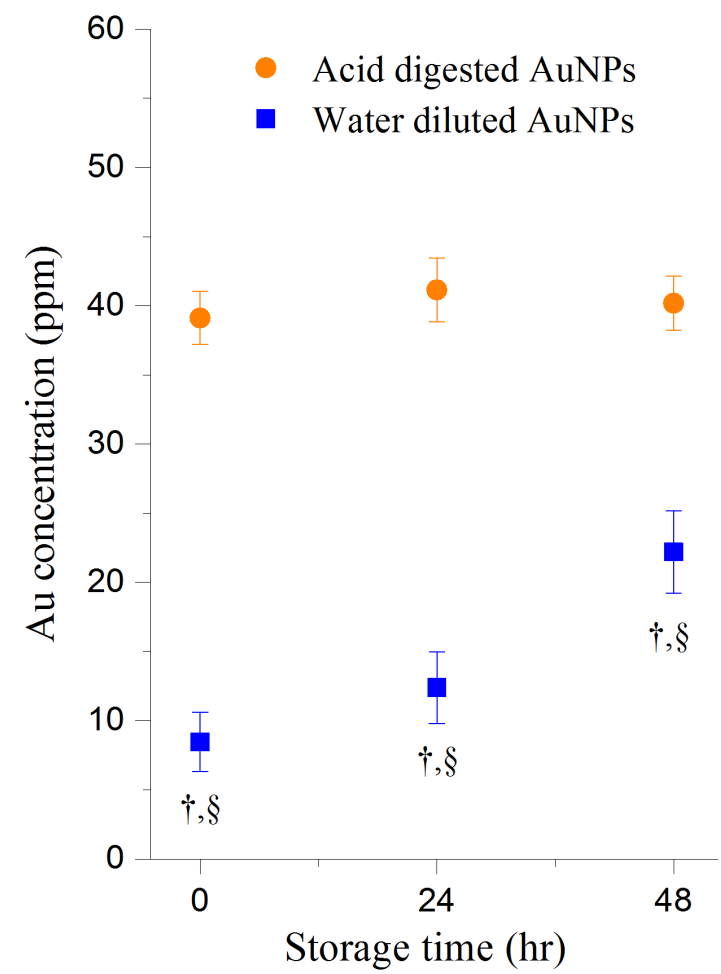

(B)

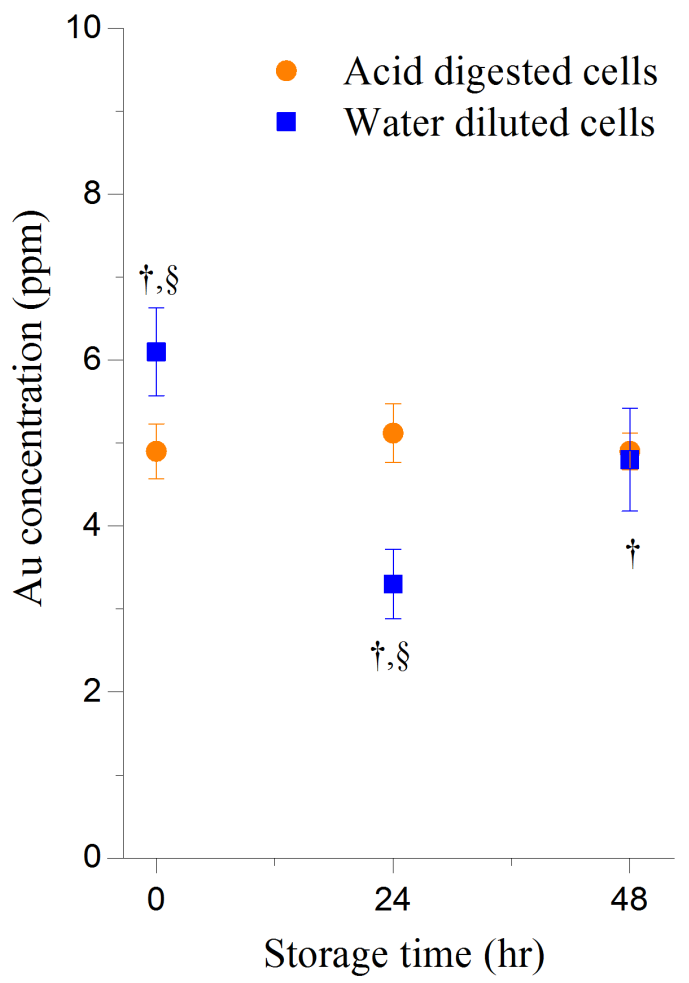

$\S \mathrm{p}<.05$, statistically significant difference between sample preparation methods.

$\mathrm{p}<.05$, statistically significant difference between sample storage time.

Figure 2-6: The effect of storage time and sample preparation methods on recovered Au concentrations. (A) Inhouse synthesized $10 \mathrm{~nm}$ AuNPs digested with 3:1 Aqua Regia and diluted with ultrapure water. (B) MDA-MB-231 cells incubated with In-house synthesized $10 \mathrm{~nm}$ AuNPs digested with 3:1 Aqua Regia and diluted with ultrapure water. The results show the obtained Au concentration with $1 \mathrm{ppm} \mathrm{La}$ as the internal standard. The error bars represent the standard deviation of concentration from using 3 separate reflectors with 3 rotations per reflector. All data was analyzed with PyMCA

As show in Figure 2-6, there is statistically significant difference in the obtained value of $\mathrm{Au}$ between water diluted and acid digested samples, both for the in-house synthesized $10 \mathrm{~nm}$ AuNPs and the MDA-MB-231 cells incubated with in-house synthesized $10 \mathrm{~nm}$ AuNPs. Moreover, the 48-hour trend of the acid digested samples shows measured Au concentration from AuNPs sample and the MDA-MB-231 incubated with AuNPs remained consistently the same over storage time. The AuNPs diluted with water resulted in seemingly increasing concentration of $\mathrm{Au}$ with storage time, while the water diluted cells resulted in decrease of concentration of $\mathrm{Au}$ followed by an increase with storage time.

Taking a closer look at the acid digested samples, the trend observed for the AuNPs and the MDA-MB-231 cells was expected. The samples were digested with acid and stored in glass 
tubes, and since neither Aqua Regia nor Au react with glass [63] the concentration was expected to remain the same with storage time. As such, the one-way ANOVA test showed there is no statistically significant difference in the obtained $\mathrm{Au}$ concentration for both acid digested samples over the 48 hours.

In order to explain why the concentration of the water diluted AuNPs increased as a function of storage time, one must examine the impact of nanoparticle aggregation [35], [83]. Naked AuNPs are prone to aggregation under various conditions such as acidity [84], temperature [85] and laser irradiation [86]. Acidity and temperature are of particular relevance to this experiment. Acid-induced aggregation of AuNPs occurs without increases in the individual nanoparticle sizes. At higher $\mathrm{pH}$, the AuNP colloids are stable as a result of the electrostatic reaction which occurs due to the oxyanion groups. This is reversed at lower $\mathrm{pH}$ causing the protonation of the anion group and the formation of uncharged hydroxy functional groups which form hydrogen bonds with nearby particles, leading to aggregation [84]. AuNP aggregation is also governed by a balance of entropic and enthalpic effects, making them very temperature dependent [85]. In fact, thermal control of nanoparticle size and morphology is used as a methodology of assembling monolayers into structured aggregates since lowering the temperature yields larger aggregates. In this experiment, the aggregation of unlabeled AuNPs as a function of storage time causes the deposited TXRF sample to contain larger AuNP aggregate units. Unlike the acid-dissolved case, these samples remain intact and as a result, the TXRF quantification technique estimates a higher concentration of Au.

In Figure 2-6 B, the starting concentration of the $\mathrm{Au}$ in the water-diluted MDA-MB-231 cell suspension was approximately $6.1 \mathrm{ppm}$. During the storage, its concentration initially dropped by $50 \%$ to $3.2 \mathrm{ppm}$ before a slight increase at $48 \mathrm{~h}$. In order to interpret this trend, one must again examine the impact of AuNP aggregation. Incubating the cells with the AuNPs at $37^{\circ} \mathrm{C}$ for 16 hours and diluting them with room temperature water prior to TXRF measurements, might have caused the AuNPs to aggregate as a result of the temperature change [85]. The subsequent drop in the estimated concentration after $24 \mathrm{~h}$ of storage suggests that the AuNPs disaggregate. However, the cause of this phenomenon was not investigated as it was beyond the scope of this work. The increase in Au concentration from 24 to $48 \mathrm{~h}$ could be a result of the equilibrium between the $\mathrm{pH}$ of the solution and the effect that storage temperature has on aggregation, similar to the one observed in the water-AuNP solutions in Figure 2-6 A. 


\subsubsection{Recovery rate of reference material AuNPs}

The importance of appropriate sample preparation method is highlighted above in section 2.3.3. However, the recovery rates of Au couldn't be calculated in section 2.3.3 as the samples were prepared with in-house AuNPs. Reference material AuNPs were thus used to further investigate the findings from section 2.3.3. The reference material AuNPs were prepared by acid digestion and water dilution. Furthermore, the acid digestion was carried out with two different ratios of Aqua Regia. The samples that were diluted with water resulted in recovery rate of $(67.5 \pm 8.1)$ $\%$, while the acid digested samples resulted in recovery rate of $(85.9 \pm 3.9) \%$ and $(102.7 \pm 3.7)$ $\%$, for the 3:1 and 4:1 Aqua Regia respectively. The undigested sample resulted in low recovery rate compared to the digested samples which is in agreement with the findings of section 2.3.3. Between the two ratios of Aqua Regia, higher recovery rate was obtained when samples were digested with 4:1 Aqua Regia (102.7 \pm 3.7$) \%$. This increased recovery rate can be attributed to the excess $\mathrm{Cl}^{-}$ions available to react with the oxidized metallic gold and form the complex ion $\mathrm{AuCl}^{-}$, therefore fully dissolving the AuNPs [87].

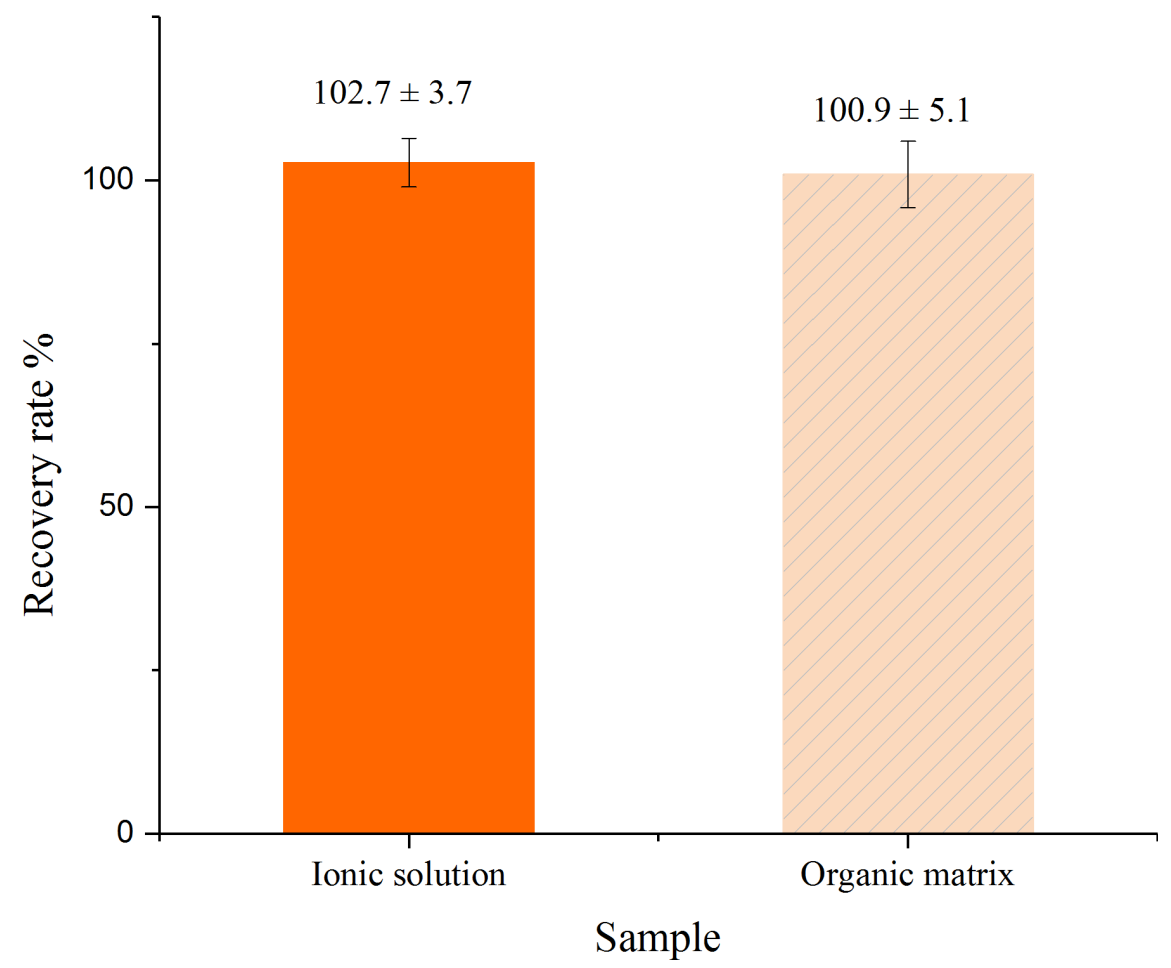

Figure 2-7: $10 \mathrm{~nm}$ reference material AuNPs digested with 4:1 Aqua Regia and $10 \mathrm{~nm}$ reference material AuNPs in the presence of suspensions of MDA-MD-231 cells digested with 4:1 Aqua Regia. The results show the obtained recovery rates with $1 \mathrm{ppm} \mathrm{La}$ as the internal standard. The error bars represent the standard deviation of recovery rates from using 3 separate reflectors with 3 rotations per reflector. All data was analyzed with PyMCA. 
The quantification method developed shows that analytically acceptable recovery rates of AuNPs can be obtained using La as an internal standard and PyMCA fitting software, provided that the samples are digested in 4:1 Aqua Regia. To validate the efficacy of this method for cellular uptake studies, $10 \mathrm{~nm}$ reference material AuNPs were mixed with MDA-MB-231 and digested with $4: 1$ acid. The obtained recovery rate of $(100.9 \pm 5.1) \%$, shows this method is capable of accurately quantifying AuNPs uptake in cancer cells.

\subsubsection{Measurement precision}

The effect of sample position on TXRF measurement precision was evaluated by rotating each reflector by $120^{\circ}$ and $240^{\circ}$ between measurements. The RSD\% was calculated for several types of samples to assess precision, where an $\mathrm{RSD} \%$ value of less than $10 \%$ is considered sufficient precision [88]. As shown in table 2-1, rotation of reflector had no effect on the precision of the measurements as all samples showed $<10 \%$ RSD $\%$. Future measurements of cellular suspensions incubated with AuNPs can thus be shortened and simplified without the need to rotate the reflector.

\begin{tabular}{cccc}
\hline Sample & Reflector 1 & Reflector 2 & Reflector 3 \\
\hline \hline $\begin{array}{c}1 \text { ppm reference Au solution } \\
\text { 1 ppm Au with 1 ppm Zn } \\
\text { reference solution }\end{array}$ & $4.5 \%$ & $2.9 \%$ & $3.2 \%$ \\
\hline $\begin{array}{c}10 \mathrm{~nm} \text { reference AuNPs } \\
\text { digested in 4:1 Aqua Regia }\end{array}$ & $1.9 \%$ & $3.4 \%$ & $3.7 \%$ \\
\hline $\begin{array}{l}10 \text { nm reference AuNPs in } \\
\text { MDA-MB-231 digested in } \\
4: 1 \text { Aqua Regia }\end{array}$ & $4.4 \%$ & $3.1 \%$ & $3.6 \%$ \\
\hline
\end{tabular}

Table 2-1:Investigating the effect of sample position on measurement precision. Precision was evaluated with RSD\%. The results show obtained RSD\% for the 3 separate reflectors used per sample. Data was analyzed with PyMCA. 


\subsection{Conclusion}

While recent developments in nanomedicine have improved conventional cancer therapies, with studies showing that only $0.7 \%$ of the systemically administered nanoparticles accumulate within the tumor, further work is still needed to increase cellular targeting and uptake. Progress of nanomedicine thus necessitates accurate and efficient means of quantitatively assessing the NP delivery in cells and tissues.

We have shown the efficacy of a TXRF based quantification method of AuNPs in breast cancer cell line. Internal standards, fitting approaches and sample preparation methods were suggested using certified reference solutions. Samples that were digested with 4:1 Aqua Regia and quantified against $\mathrm{La}$ as the internal standard accompanied with PyMCA fitting software showed analytically acceptable recovery rates of $(102.7 \pm 3.7) \%$ and $(100.9 \pm 5.1) \%$. Sample storage time was also investigated, and the results suggest that AuNPs and MDA-MB-231 cells incubated with AuNPs can be stored up to $48 \mathrm{hrs}$ after acid digestion without affecting measurement precision. Using reference material AuNPs, this study showed that AuNPs in organic matrix can be measured accurately and precisely. The simple sample preparation steps and small sample volume $(5 \mu 1)$ this method requires makes it a valuable alternative to ICP based methods.

\subsection{Acknowledgements}

The authors would like to thank Dr. Devika Chithrani (Department of Physics and Astronomy, Faculty of Science, University of Victoria) and Celina Yang (Department of Physics, Faculty of Science, Ryerson University) for generously providing the cell cultures and in-house synthesized AuNPs. Diane Eichert (Elettra-Sincrotrone Trieste) is also kindly thanked for her assistance with PyMCa. This work was supported by the Natural Sciences and Engineering Research Council Research tool and instruments grant and discovery grant (APM), and the Queen Elizabeth II Graduate Scholarships in Science and Technology (GM). Ryerson University's department of Physics is also acknowledged for the financial support of this work. 


\subsection{Conflict of interest disclosure}

The authors have no conflict of interest to declare. 


\section{COMPARISON OF TXRF QUANTIFICATION OF GOLD NANOPARTICLES WITH ICP-AES - PRELIMINARY INVESTIGATIONS}

\subsection{Introduction and chapter organization}

The ability of TXRF to quantify AuNPs within organic matrix was demonstrated in chapter 2. Using well-characterized suspensions of reference material AuNPs, it was demonstrated that TXRF technique based on La as an internal standard and PyMCA deconvolution of the Au- $\mathrm{Zn}$ overlap is able to yield recovery rate of $100.9 \pm 5.1 \%$. This chapter will compare the conventionally used technique of ICP-AES (introduced in section 1.4.1.3) against the TXRF method developed in chapter 2 for quantifying AuNPs inside MDA-MB-231 breast cancer cells. Given the global prevalence of breast cancer in women, MDA-MB-231 cell line poses a great significance in cancer research. The overarching goal of this chapter is twofold:

1. To use a well-controlled, in-vitro experiment to assess the performance of TXRF against the conventional standard in analytical spectroscopy, namely ICP-AES.

2. To demonstrate the advantages of TXRF over ICP-AES as it pertains to sample preparation.

The chapter is organized as follows. First, the materials and methods utilized for the comparative experiments is summarized. The results include the direct comparison between the TXRF and ICP-AES recovery rates for reference material AuNPs in the presence of organic matrix. Further, the comparison in gold concentration estimates for synthesized AuNPs using the two techniques are summarized. Finally, the effects of sample preparation for gold quantification in incubated MDA-MB-231 cells are presented. A discussion of the experimental results will be followed by a conclusion of the findings of this work. 


\subsection{Materials and methods}

\subsubsection{Cellular suspensions and nanoparticle synthesis}

MDA-MB-231 breast cancer cells derived from mammary gland were purchased from ATCC (Manassas, VA, USA). Suspensions were maintained in Dulbecco's Modified Eagle's Medium supplemented with $10 \%$ fetal bovine serum at $37^{\circ} \mathrm{C}$ with $5 \% \mathrm{CO}_{2}$. The cells were grown to $80 \%$ confluency then were either incubated with synthesized $10 \mathrm{~nm}$ AuNPs or exposed to the same 10 nm NIST reference AuNPs used in chapter 2.

The in-house AuNPs (referred to as AuNPs hereinafter) were synthesized using the Turkevich method described in section 1.1.1 and refined by Chithrani and colleagues [28]. Briefly, $300 \mu \mathrm{l}$ of $1 \%$ chloroauric acid (Sigma-Aldrich Corporation, St. Louis, MO, USA) was added to $30 \mathrm{ml}$ of double distilled water prior to heating on a hot plate while stirring. Upon reaching its boiling point, $1 \mathrm{ml}$ of $1 \%$ sodium citrate tribasic dehydrate (Sigma-Aldrich) was added to form nanoparticles of $10 \mathrm{~nm}$ diameter. After the solution changed color from dark blue to red, the solution was kept boiling for 5 minutes before allowing the solution to cool to room temperature, while continuously stirring. The size of the resulting AuNPs was also independently verified using transmission electron microscopy. While it was shown that $50 \mathrm{~nm}$ AuNPs lead to higher accumulation inside mammalian cells under in-vitro [28], [34], [37], [89], this experiment studied the uptake of $10 \mathrm{~nm}$ AuNPs. This was due to the fact that smaller NPs have been shown to be more feasible for targeting the nucleus and penetrating through the nuclear pore complex described in section 1.3.1 [27]. Additionally, smaller NPs have also been shown to lead to deeper tumor penetration and thus enhanced therapeutic effect [20], [90]. The $10 \mathrm{~nm}$ diameter NIST reference AuNPs (referred to as NIST AuNPs) were purchased from the National Institute of Standard and Technology or NIST (Gaithersburg, MD, USA) and were used without any modifications.

The cells were incubated with $0.3 \mathrm{nM} 10 \mathrm{~nm}$ AuNPs for 16 hours prior to being washed with Phosphate Buffered Saline (PBS) three times. Given the collaborative nature of this project, the optimization of the incubation times was chosen by collaborators for experiments outside the scope of this thesis. Following the PBS wash, the adherent MDA-MB-231 cells were dissociated from the culture dish using a solution of $0.25 \%$ trypsin-EDTA. By comparison, the NIST 
samples were not incubated with the cells but rather simply introduced to a post-trypsinization suspension of cells. In total, triplicate solutions of the following samples measured with both ICP-AES and TXR are shown in table 3.1 along with the rationale for measuring each sample. A total of 36 reflectors were used for measuring all the samples with TXRF while 36 measurements were performed with ICP-AES.

\begin{tabular}{|c|c|}
\hline $\begin{array}{l}\text { Sample description } \\
\text { (Abbreviation) }\end{array}$ & Rationale for using the sample \\
\hline $\begin{array}{l}\text { Cells with NIST reference AuNP } \\
\text { suspension } \\
\text { (MDA-MB-231-NIST AuNPs) }\end{array}$ & $\begin{array}{l}\text { To estimate the recovery rates of TXRF/ICP-AES } \\
\text { using AuNPs with a known concentration in the } \\
\text { presence of the organic matrix from cells. Other than } \\
\text { the work of Chithrani et al in } 2006 \text { [89], no other } \\
\text { publications had examined the development of ICP- } \\
\text { AES as a method for AuNP uptake inside cells. } \\
\text { However, that work did not investigate recovery rates } \\
\text { since the initial concentration of the nanoparticles } \\
\text { prior to incubation was not known. Using the } \\
\text { reference NIST AuNPs with a known certified } \\
\text { concentration, the absolute recovery rate of both ICP- } \\
\text { AES and TXRF are obtained. }\end{array}$ \\
\hline $\begin{array}{l}\text { In-house AuNPs alone } \\
\text { (AuNPs) }\end{array}$ & $\begin{array}{l}\text { To obtain the initial concentration prior to incubation } \\
\text { with cells. This is required to understand how many of } \\
\text { the administered AuNPs are taken up by the cells. }\end{array}$ \\
\hline $\begin{array}{l}\text { Cells incubated with in-house AuNPs } \\
\text { (MDA-MB-231-AuNPs } \\
\text { boiled/un-boiled) }\end{array}$ & $\begin{array}{l}\text { To investigate ability of the newly developed TXRF } \\
\text { method for accurately quantifying internalized NPs } \\
\text { inside cells. Since the ICP-AES (but not TXRF) } \\
\text { requires boiling of the samples prior to measurements } \\
\text { (see section 1.4.1.3), the incubated cell samples were } \\
\text { measured with and without boiling. }\end{array}$ \\
\hline
\end{tabular}

Table 3-1: Summary of the samples measured with TXRF and ICP-AES. 


\subsubsection{ICP-AES: sample preparation and measurements}

The ICP-AES was performed using an Optima 7300 DV system (Perkin Elmer, Waltham, MA, USA). All calibration curves were prepared using certified reference solutions supplied by the Department of Chemistry at the University of Toronto Analytical Laboratory for Environmental Science, Research and Training. All samples were prepared in triplicates and each replicate was measured three times. The output of the ICP-AES was a concentration of the Au atoms in parts per million (ppm). The size of the spherical AuNP was used to estimate the number of nanoparticles per cells taking into account the number of MDA-MB-231 cells per sample. Equations 3.1 - 3.3 provide an expression for estimating the number of AuNPs per cell [89]. These equations were also applied for the TXRF estimations.

$$
\frac{\text { Number of Au atoms }}{\mathrm{AuNP}}(\mathrm{U})=\frac{2}{3} \pi\left(\frac{\mathrm{D}}{\mathrm{a}}\right)^{3}
$$

where, $D$ is the diameter of the AuNP $(10 \mathrm{~nm})$ and a is the length of a unit cell $(0.408 \mathrm{~nm})$

$$
\frac{\text { Number of AuNPs }}{\text { Sample }}=\mathrm{C}_{\mathrm{Au}} * \mathrm{~V}_{\text {sample }} * \frac{1}{\mathrm{M}_{\mathrm{Au}}} * \mathrm{~N}_{\mathrm{A}} * \frac{1}{\mathrm{U}}
$$

where, $\mathrm{C}_{\mathrm{Au}}$ is the measured concentration of $\mathrm{Au}, \mathrm{V}_{\text {sample }}$ is the sample volume, and $\mathrm{M}_{\mathrm{Au}}$ is the molar mass of $\mathrm{Au}$.

$$
\frac{\text { Number of AuNPs }}{\text { Cell }}=\frac{\text { Number of AuNPs per sample }}{\text { Number of cells per sample }}
$$

The sample preparation prior to the ICP-AES measurements depended on sample measured. The MDA-MB-231-NIST AuNP samples was prepared by mixing $1 \mathrm{ml}$ of MDA-MB231 suspension with $1 \mathrm{ml}$ of NIST AuNPs and $1 \mathrm{ml}$ of aqua regia (4:1 mixture of hydrochloric acid and nitric acid). The samples were boiled in $200^{\circ} \mathrm{C}$ silica bath for 2 hours. The boiling procedure was replicated from previous studies quantifying AuNPs using ICP-AES [1]-[5],[8]. Any undissolved solids were removed through a filter. The remaining sample was then diluted to $5 \mathrm{ml}$ using Milli-Q water (EDM Millipore, Billirica, MA, USA).

The AuNPs ( $1 \mathrm{ml}$ volume) solutions were dissolved in a 4:1 aqua regia mixture. They were however not boiled as there is no organic matrix present thus no sample digestion is necessary [72]. In contrast, the $1 \mathrm{ml}$ of cells incubated with AuNP for 16 hours (MDA-MB-231AuNPs) were digested with $4: 1$ aqua regia and boiled in a $200^{\circ} \mathrm{C}$ silica bath for 2 hours. Any undissolved solids in the suspension were removed through filtration. The filtered samples were 
diluted to $5 \mathrm{ml}$ using Milli-Q water. The un-boiled samples were prepared in an identical fashion as described above, however, without the silica bath boil. These samples were prepared in order to investigate the effect of sample preparation on the recovery of AuNPs.

\subsubsection{TXRF: sample preparation and measurements}

The S2 PicoFox TXRF spectrometer (Billirica, MA, USA) was also utilized for these measurements. The reflectors were siliconized using a $10 \mu \mathrm{l}$ silicone solution $(1: 500 \mathrm{v} / \mathrm{v}$ Sigmacote ${ }^{\circledR}$ to $\left.n-H e x a n e\right)$. They were subsequently transferred to an oven (Boekel Scientific, Festerville, PA, USA) where they were dried for 30 minutes at $80^{\circ} \mathrm{C}$. Blank TXRF measurements of each reflector were then taken to ensure the absence of contaminants. The La internal standard was prepared from a certified reference solution (VWR International, Radnor, PA, USA) diluted with Milli-Q water. The internal standard was added to each sample and mixed to form a homogenous sample. A volume of $5 \mu \mathrm{l}$ was then deposited into the siliconized reflector and it was dried in the $80^{\circ} \mathrm{C}$ for 20 minutes. These procedures were identical regardless of the samples measured. Each sample was prepared in triplicates and each triplicate was measured three times (matched with ICP-AES). Each measurement was conducted for 900 seconds. All the acquired spectra were analyzed using the PyMCA software (European Synchrotron Radiation Facility, Grenoble, France). All the analysis techniques described in chapter 2 using standard reference solutions were applied for estimating the Au concentration in these samples as well. Just like the ICP-AES measurements, the recovery rate from MDA-MB-231-NIST AuNP samples were computed along with the number of AuNPs per cell for both boiled and un-boiled samples. These metrics were compared directly with the estimates of ICP-AES for every sample.

\subsection{Results and Discussion}

\subsubsection{Recovery rates for reference material nanoparticles in presence of organic matrix}

As shown in chapter 2, the use of NIST reference gold nanoparticles demonstrated that TXRF is capable of accurately quantifying AuNPs in the presence of organic matrix (i.e. cellular components from cancer cells) with recovery rate of $100.9 \pm 5.1 \%$. Chithrani and colleagues have shown that ICP-AES is also capable of quantifying various sizes and shapes of AuNPs in 
the presence of cells [89]. It was reported that approximately 3000 spherical AuNPs (14 nm diameter) were localized inside HeLa cells after 6 hours of incubation. However, the recovery rate of the ICP-AES protocol used in this seminal study (or subsequent follow-up studies) was not investigated. A subsequent study by Fischer et al reported using aqueous standards with certified concentrations that the recovery of Au after nitric acid digestion (in combination with liver tissue homogenate) was $85 \pm 12.6 \%$ [91]. To the best of our knowledge, the recovery rate of ICP-AES using certified gold nanoparticles has never been reported in the literature.

Figure 3-1 shows the comparison between the recovery rates of NIST AuNPs in the presence of MDA-MB-231 cells measured with TXRF and ICP-AES. The recovery rate of TXRF is $94.6 \pm 3.4 \%$ while that of ICP-AES is $44.8 \pm 2.4 \%$. In calculating the recovery rates, the sample was first measured with ICP-AES and the remaining sample was measured with TXRF. This approach reduced the possibility of discrepancy from measuring different samples and allowed for a direct comparison. As shown from the results in chapter 2 (section 2.3.4) using NIST AuNPs (alone and with cells), the recovery of TXRF was nearly $100 \%$. This is in agreement with results from Chapter 2 and Figure 3-1 implies that TXRF results are reproducible across multiple experimental conditions (ex. cell passing, numbers and days). 


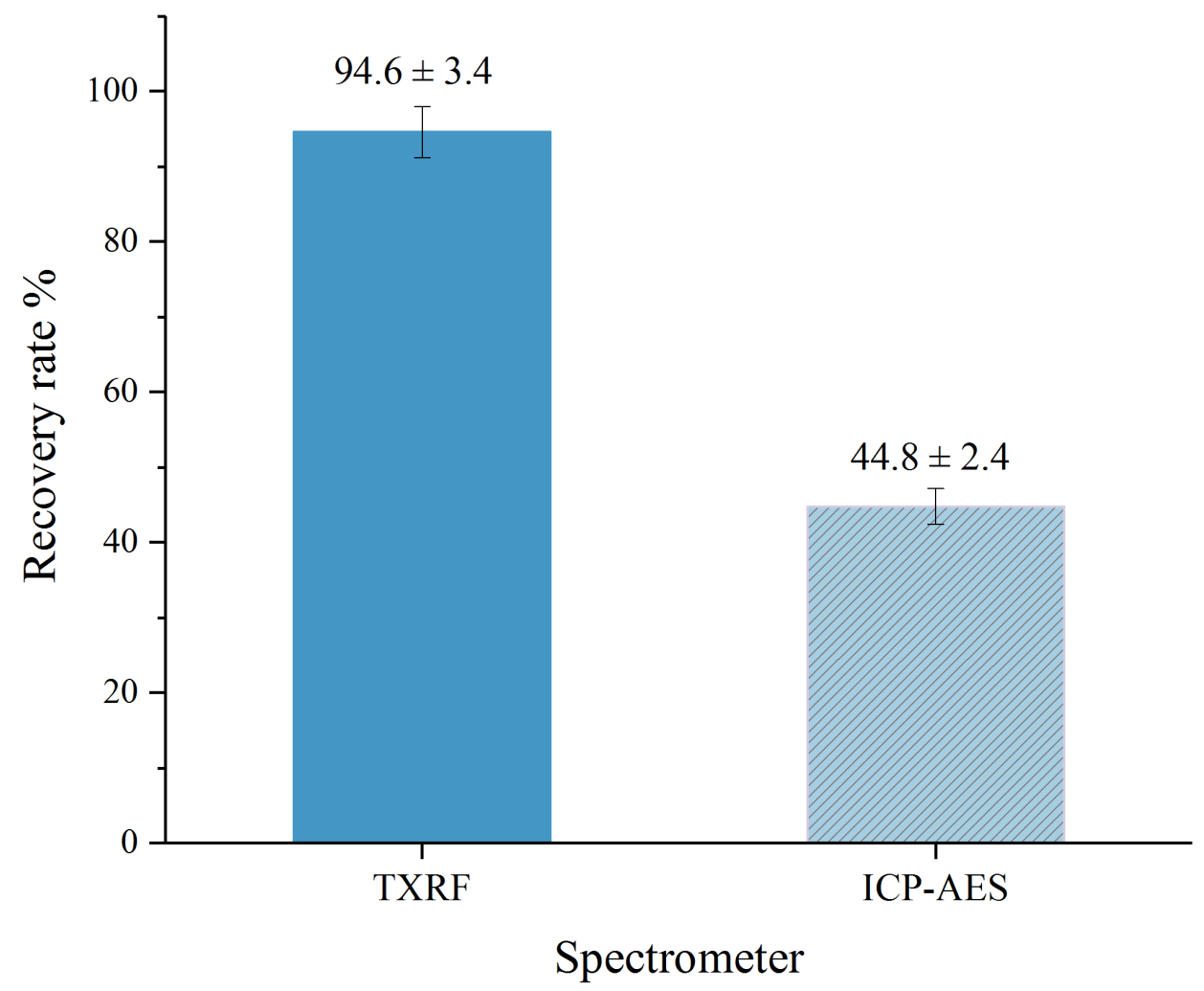

Figure 3-1: Comparison of the TXRF and ICP-AES recovery rates of $10 \mathrm{~nm}$ NIST AuNPs in the presence of a suspension of MDA-MB-231 cells. The error bars for TXRF represent the standard deviation from recovery rates from using 3 separate cell suspensions with 3 separate reflectors each. The error bars for ICP-AES represent the standard deviation of triplicate measurements for 3 separate cell suspensions.

The low ICP-AES recovery rate suggests that the sample preparation plays an important role. During the ICP measurements, the sample sucked into the nebulizer must be in aqueous form [52], [59]. For biological samples, this is achieved through acid digestion which completely dissolves the organic matrix present in cells. The 2-hour boiling implemented in this experiment as well as other work reported in literature might only partially digest the matrix. It is possible that the organic matrix made it to the filtered solution, and subsequently to the plasma torch resulting in matrix interference. Insufficient boiling time can also result in matrix mismatch between the sample and the standard solutions used for the calibration curve. Most ICP-AES applications dealing with biological samples make use of elevated pressures or microwave digestions to achieve higher temperatures for maximal digestion [52]. Sample preparation has also been reported to affect ICP-MS results and the quantification of various types of nanoparticle suspensions when multiple methods of digestion (acid or microwave) are employed [74]. 


\subsubsection{ICP vs. TXRF: Comparisons in estimates of Au concentration}

Figure 3-2 shows the comparison between the concentration of Au when the in-house AuNPs were measured in the absence of any organic matrix. A significant difference (45\%) exists between the quantification obtained by the two techniques with TXRF estimating a higher concentration of Au than ICP-AES. This sample simply consists of solutions of the AuNPs synthesized with the Turkevich method [2], which have not been boiled after acid digestion. The goal of this measurement was to obtain the initial concentration of AuNPs prior to incubation with cells.

As reported by Allabashi et al, ICP-MS is capable of estimating the concentrations of $\mathrm{Au}$ without prior digestion of AuNPs in the absence of organic matrix [72]. Thus, it was expected that both techniques would yield similar estimates in Au concentration since there is no organic matrix present. The obtained results however show contrary to what was expected. It's important to note that Allabashi et al had samples that were $1 \%(\mathrm{v} / \mathrm{v})$ hydrochloric acid, which matched the matrix of the calibration solutions [72]. One can thus speculate that potential reasons for the low $\mathrm{Au}$ concentrations obtained by ICP-AES might lie in matrix mismatch between samples and the calibration curve. A proper calibration curve preparation and matrix matching is paramount to the accuracy of ICP-based techniques for estimating analyte concentrations [52], [56]. As discussed in section 1.4.1.4 and highlighted in section 1.4.1.5, matrix effect can substantially influence recovery rates. TXRF on the other hand shows exceeding capabilities in estimating the analyte concentrations. The difference in concentration of Au obtained between ICP-AES and TXRF is consistent with the difference in recovery rate observed between the two techniques shown in Fig 3.1, which suggests TXRF based approach was able to accurately quantify the concentration of the in-house AuNPs. 


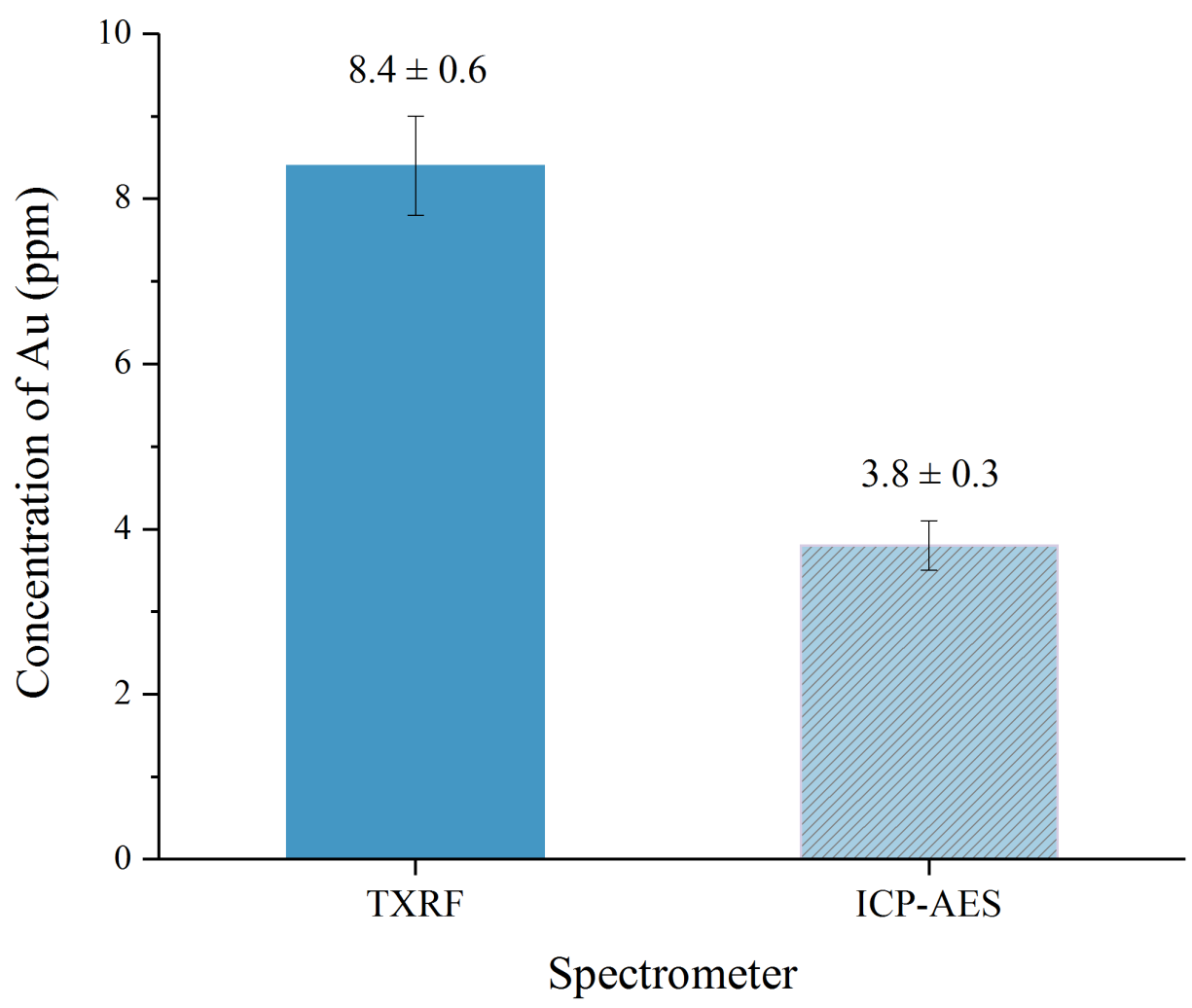

Figure 3-2: Comparison of the estimated Au concentration from synthesized $10 \mathrm{~nm}$ AuNP suspensions. The error bars for TXRF represent the standard deviation from recovery rates from using 3 separate cell suspensions with 3 separate reflectors each. The error bars for ICP-AES represent the standard deviation of triplicate measurements for 3 separate cell suspensions.

The next step was to investigate the ability of the newly developed TXRF method to accurately quantify AuNPs taken up by cells. The effect of sample boiling was also examined in order to further investigate the impact that sample preparation has on estimates of $\mathrm{Au}$ concentration. Figure 3-3 shows a representative TXRF spectrum from a suspension of MDA-MB-231 cells incubated with in-house Au-NPs. The overlap of Au peak from the in-house nanoparticles with the $\mathrm{Zn}$ present in the cells (shown by the $\mathrm{K}_{\beta}$ ) and as well as other peaks of interest are shown. 


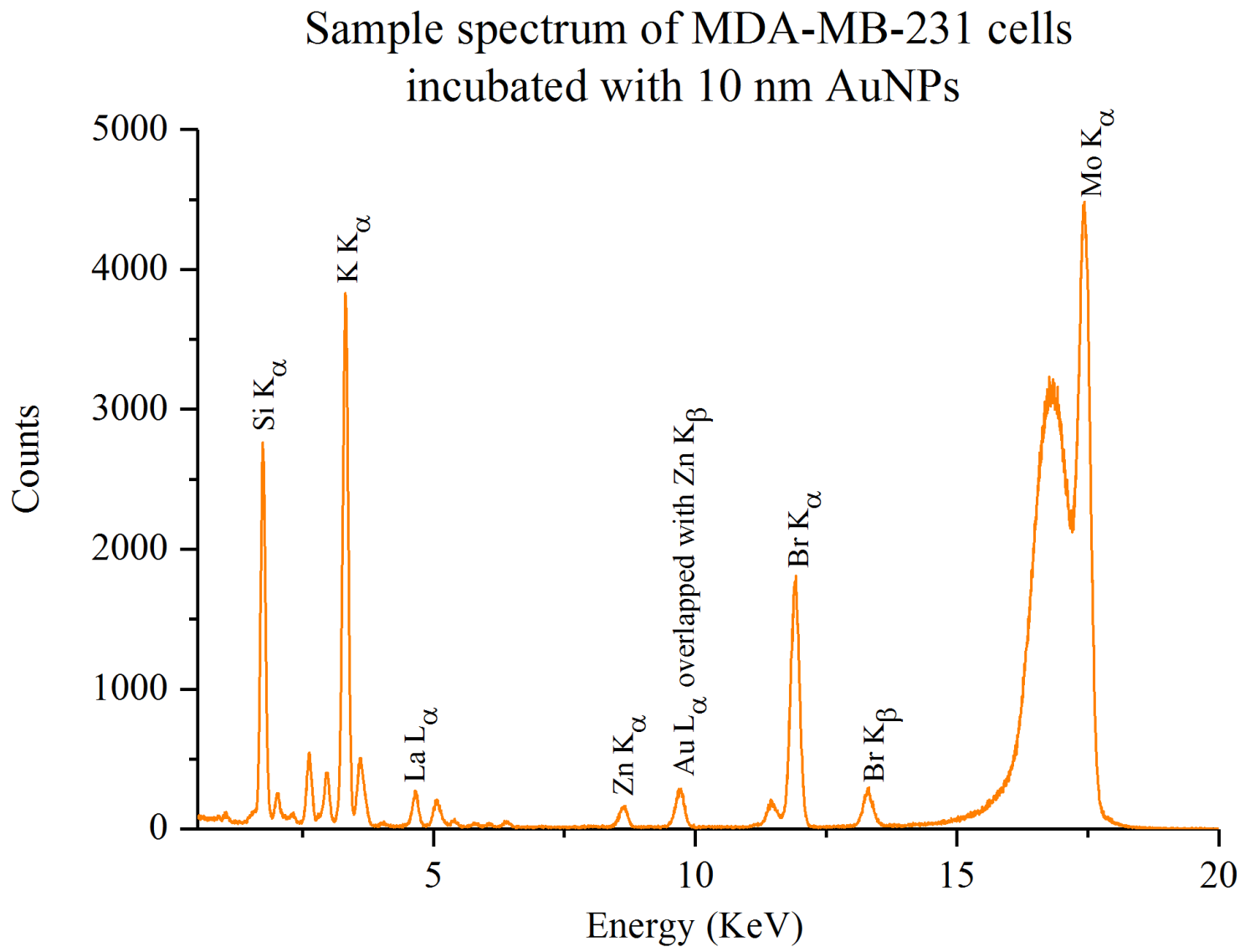

Figure 3-3: Representative TXRF spectrum from MDA-MB-231 cells incubated with 10 nm AuNPs for 16 hours. The spectrum shows the relevant peaks of interest as a result of the X-ray tube (Mo), sample reflector (Si), internal standard (La) and the AuNPs overlapping with the endogenous $\mathrm{Zn}$ present inside cells.

The deconvolution method developed and validated in chapter 2 was used to quantify the uptake of $\mathrm{Au}$ within cells incubated for 16 hours. Using the concentrations of Au measured and equations 3.1 - 3.3, one can compute the number of AuNPs inside MDA-MB-231 cells. Figure 3-4 shows the estimated number of AuNPs per cell from both TXRF and ICP-AES; boiled and un-boiled samples are shown for both techniques. It is evident that ICP-AES estimates are approximately 50\% lower than TXRF for both conditions - consistent with the results shown in Fig 3.1 and 3.2. This result can potentially be explained by the combination of possible incomplete digestion of sample and the complex matrix matching requirements of ICP-AES.

The difference between the two techniques observed in Figure 3-4 is consistent with that observed in Figure 3-1, where the NIST AuNPs were quantified in an MDA-MB-231 suspension. Given the high recovery obtained in Fig 3.1 (94.6\%), the consistent difference suggests that TXRF based approach was able to accurately quantify AuNPs uptaken by the breast 
cancer cells. The results also show boiling a sample prior to TXRF measurements does not make a difference in estimating the number of internalized nanoparticles per cell.

Given that no difference was observed between boiled and un-boiled samples for ICPAES, this provides further evidence that the 2-hour silica bath boiling is not sufficient for matching the sample matrix with that of the calibration curve, subsequently affecting the obtained recovery rate. Wet ashing of organic materials results in strongly acidic solutions. The solution must either be diluted with large amount of base or digestion must be continued longer to drive of excess acid [65]. Dilution is not a viable option when dealing with samples that have low starting concentration of analyte. Thus, samples need to be further digested to match the matrix of the calibration stands. This could also explain the low recovery rate of obtained by ICP-AES for the reference material (NIST AuNP) reported in section 3.3.1.

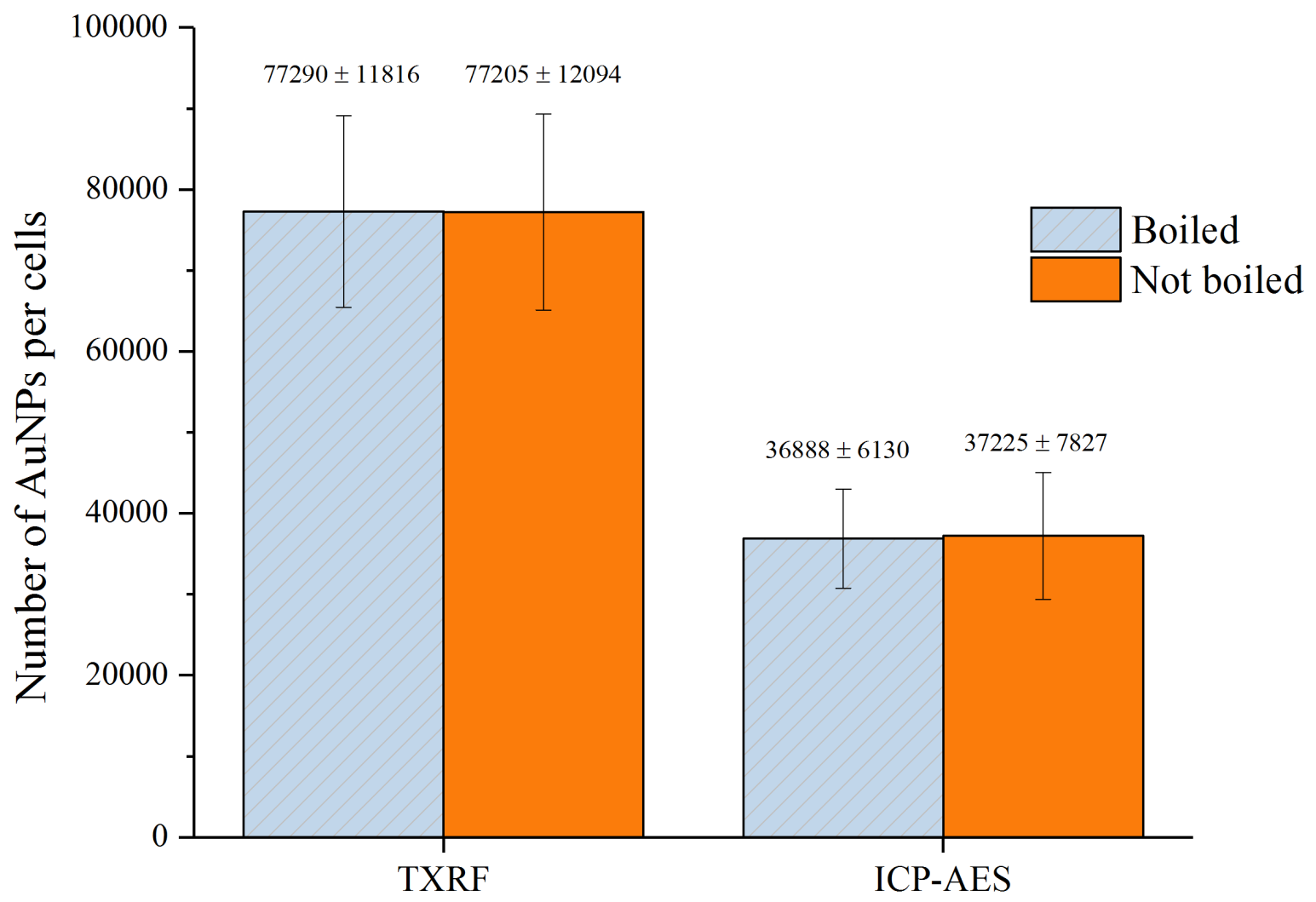

Spectrometer

Figure 3-4: Comparative estimates of the number of AuNP per cell using TXRF and ICP-AES for boiled and unboiled samples of MDA-MB-231 cells incubated with AuNPs. Incubation time was 16 hours. The error bars for TXRF represent the standard deviation from using 3 separate cell suspensions with 3 separate reflectors each. The error bars for ICP-AES represent the standard deviation of triplicate measurements for 3 separate cell suspensions. 
The $50 \%$ difference in recovery rate measured by both techniques for the same sample carries across all samples. This suggests that the sample preparation for ICP-AES quantification of Au-NPs plays a crucial role in accurately estimating Au concentration. Further investigations of sample preparation are required in order to understand the limitations of ICP-based techniques for the AuNP quantification. Most importantly, the high recovery of TXRF strongly suggests that the technique can indeed quantify the amount of Au incubated inside a cell.

\subsection{Conclusions}

This chapter demonstrates for the first time a direct comparison between TXRF and ICP-AES for the quantification of AuNPs inside breast cancer cells. Measurements of reference material AuNP from NIST resulted in recovery rates of $44.8 \pm 2.5 \%$ and $94.6 \pm 3.4 \%$ for ICP-AES and TXRF respectively. The discrepancy between the ICP-AES and TXRF obtained concentration were consistent for all samples, suggesting the importance of proper ICP-AES sample preparation for accurate quantification. The results also showed the newly developed TXRF method can accurately quantify uptake of AuNPs by breast cancer cells without the need to digest the organic matrix, thereby allowing for simple and fast sample preparation. 


\section{THESIS CONCLUSIONS AND FUTURE WORK}

\subsection{Thesis conclusions}

The focus of this thesis was to assess the feasibility of TXRF to quantify the uptake of AuNPs within breast cancer (MDA-MB-231) cell cultures. All thesis objectives outlined in chapter 1 (section 1.6.2) were investigated. This work demonstrated, for the first time, that the AuNP concentration in organic matrix can be accurately recovered (90-110\% recovery rate) using TXRF.

Using certified reference solutions, it was shown that La is a suitable internal standard for AuNP quantification and that PyMCA fitting can provide analytically acceptable recovery rates. Comparisons with $\mathrm{Y}$ as the internal standard using certified reference Au solutions revealed that $\mathrm{La}$ is a more suitable element for the external fitting models. The $\mathrm{Zn}$ that is naturally present in this cell culture presented challenges due to the proximity of the Zn's K $\beta$ and Au's L $\alpha$ peaks. By investigating varying concentrations of $\mathrm{Zn}$ relative to $\mathrm{Au}$ in the sample, it was shown that PyMCA is a suitable fitting software for the deconvolution of the overlapped $\mathrm{Zn} / \mathrm{Au}$ peaks.

Sample preparation approaches were also investigated and it was found that aciddigestion prior to measurements yields samples which meet the TXRF thickness criterion in the presence of the cellular organic matrix. Using in-house AuNPs, the effect of storage time was also investigated in the presence and absence of cells. This experiment shed light into the effects of AuNP aggregation and solution $\mathrm{pH}$ and the impact that this has on TXRF-estimated concentrations. Shelf life is an important parameter in analytical measurements and these results demonstrate that acid digested samples can be stored up to $48 \mathrm{hrs}$. without affecting TXRF measurement.

The validity of the internal standard choice, fitting approach and sample preparation was assessed using NIST reference material AuNPs. The results presented in chapter 2 (section 2.3.4) revealed that recovery rates of AuNPs (without cells) was (102.7 \pm 3.7$) \%$. Mixing the cells with the reference material AuNPs and digesting the sample in 4:1 Aqua Regia yielded an acceptable recovery rate of $(100.9 \pm 5.1) \%$. These results indicated that TXRF can accurately quantify AuNPs provided that La is used as the internal standard equipped with PyMCA fitting approach, and that samples are digested with Aqua Regia. This protocol provides a building block in testing 
the feasibility of TXRF for the quantification of nanoparticles in the presence of cellular material.

A direct comparison of the TXRF quantification of AuNPs with the conventional method of ICP-AES post-incubation was performed as shown in chapter 3 (section 3.3). In-house and NIST reference AuNPs were incubated with MDA -MB-231 cells and quantified using the method developed in chapter 2 (section 2.3.4). TXRF outperformed ICP-AES by nearly 50\% in estimating the NIST AuNPs as well as the number of nanoparticles per cell after 16 hours of incubation. The discrepancy between the two techniques was attributed to the sample preparation methodology employed for ICP-AES, particularly matching the sample matrix to that of the calibration curve. The findings of this thesis demonstrate the capability of TXRF to accurately quantify the AuNP uptake in cancer cells with minimal sample preparation.

\subsection{Future work}

The results presented in this thesis are the first-ever investigations to demonstrate that TXRF can accurately (90-110\% recovery rate) quantify uptake of AuNPs in mammalian cancer cells. In order to further explore the potential of the developed method for quantifying uptake of nanoparticles in biological samples, several investigations are required. They are summarized below:

1. Optimization of TXRF measurement time: In this thesis, each sample was measured for 600 seconds to ensure adequate counting statistics for acceptable recovery. It would be worthwhile to investigate the lowest measurement time that can yield $90-110 \%$ recovery rate. These experiments can be conducted using certified reference solutions and cells incubated with reference material nanoparticles. Lowering the "per sample measurement time" would allow TXRF to measure multiple experimental conditions, which is typical for nanoparticlebased research [34], [42]. Additionally, this optimization is necessary for estimating the cost of operation and maintenance of TXRF equipment.

2. Direct comparisons of TXRF with ICP: Chapter 3 (Section 3.3) showed a preliminary investigation which compared the recovery rates of ICP-AES with TXRF for nanoparticle quantification. The sample preparation issues highlighted in this section (ex. longer boiling time for matching the sample matrix) and their impact on recovery rates suggest that further investigations are required in order to optimize the ICP quantification. Proper digestion of the 
organic matrix is crucial for obtaining suitable recoveries; acid digestion and sample boiling protocols must be carefully investigated. Specifically, microwave digestion approaches for dissolving the organic matrix common in ICP-related studies [72], [74], [91] should be considered. Any and all preparation steps taken for ICP must also be applied to TXRF in order to ensure direct comparison between the two techniques is achieved. This comparison will further strengthen TXRF' standing as a robust nanoparticle quantification tool and expand upon current existing applications in the field [67].

\section{Extend the developed TXRF quantification method to other nanoparticle and cell line} combinations: As introduced in chapter 1 (section 1.2), a diverse population of nanoparticles are currently in use for biomedical research due to their versatility [1], [5]. To test the capabilities of the developed technique for quantifying nanoparticle uptake in cancer cell suspensions, commonly used nanoparticles such as $\mathrm{Pt}$ or magnetic $\mathrm{Fe}_{2} \mathrm{O}_{3}$ can be investigated with the current spectrometer. Pt has a high atomic number and like Au it can be used in radiotherapy. It has been shown that hadron radiation with PtNP showed a 2-fold increase in DNA damages [92]. Additionally, PtNPs have also been used as a electrochemical biosensor to improve the electro-activity for hydrogen peroxide and glucose [93]. $\mathrm{Fe}_{2} \mathrm{O}_{3} \mathrm{NPs}$ have been used as to enhance the contrast in magnetic resonance imaging due to their superparamagnetic properties which can shorten the T2 water relaxation time [94]. Magnetic nanoparticles do not exhibit the harmful effects of gadolinium, especially in patients with weakened renal function [95].

Examining the uptake of these nanoparticles in various cell lines would be of interest for various biomedical applications. For instance, the radiosensitization properties of $\mathrm{HfO}_{2} \mathrm{NPs}$ have been demonstrated in-vitro in HTC116 colorectal carcinoma cells and in-vivo in tumor xenografts [96]. Given that NPs can also cause thermal induced damage to cellular components [97], it is imperative that the kinetics of their uptake is quantified in relevant biomedical applications where multiple other types of cancer cell lines (ex. prostate, kidney, skin) are utilized [98]. This will diversify the capabilities of TXRF as a quantification technique. It is worth noting that the diverse choices for NP material do not necessarily allow themselves for TXRF quantification. For instance, carbon nanotubes are becoming more and more utilized in biomedical imaging [99] and cancer therapy [100] without influencing the activity of the immune system [101], a significant feat in reducing systemic cytotoxicity. Due 
to the low-energy $(<0.5 \mathrm{KeV})$ peak of carbon, TXRF quantification of the uptake of carbonbased nanoparticles is simply not feasible with the system described in this thesis. This is a potential limitation of the technique which requires that the elemental fluorescence peaks be located within the detector energy bandwidth (1-20 keV) and not overlap with other permanent peaks within the system such as $\operatorname{Ar}$ (air), Si (reflector) and Mo (tube) [67].

\section{Quantify nanoparticle uptake in excised tumor samples and tissues from in-vivo} experiments: Given that one of the goals of nanoparticle research is the clinical translation for cancer therapy, understanding their uptake in a realistic (in-vivo) tumor environment is important [42]-[44]. Excising of tumor samples is standard practice in cancer research as histology is a widely used means of examining the biological environment of the tumor [48]. Excised tumor samples make for a suitable biological model for quantifying nanoparticle uptake with TXRF. Previous work on the quantification of elemental concentrations in breast [102] and prostate [103] cancerous tissue with synchrotron based X-ray fluorescence methods has been reported. Translating these studies to a bench top TXRF requires refinement of the sample preparation and internal standard quantification methods developed for cellular suspensions in order to meet the needs of excised tissue samples, particularly as it pertains to meeting the TXRF thickness criteria.

Another important aspect of nanoparticle research in-vivo is the bio-distribution [47] and pharmacokinetics [104] of uptake as they become important factors in assessing cytotoxicity [105], [106]. TXRF quantification of nanoparticle distributions in various tissues would be of great interest for researchers designing targeted agents. This can be achieved by extracting normal and cancerous tissues samples from animals injected with NPs, and measuring them as micrometer thin histological specimens. The histological measurements would allow the TXRF-based quantification to be correlated with electron microscopy images of the sections, providing a direct metric of the biodistribution of NPs in various sites of interest. 


\section{REFERENCES}

[1] C. Rancoule et al., "Nanoparticles in radiation oncology: From bench-side to bedside," Cancer Lett., vol. 375, no. 2, pp. 256-262, Jun. 2016.

[2] J. Turkevich, P. C. Stevenson, and J. Hillier, "A study of the nucleation and growth processes in the synthesis of colloidal gold," Discuss. Faraday Soc., vol. 11, no. 0, pp. 5575, Jan. 1951.

[3] G. Frens, "Controlled Nucleation for the Regulation of the Particle Size in Monodisperse Gold Suspensions," Nature, vol. 241, no. 105, pp. 20-22, Jan. 1973.

[4] I. H. El-Sayed, X. Huang, and M. A. El-Sayed, "Surface plasmon resonance scattering and absorption of anti-EGFR antibody conjugated gold nanoparticles in cancer diagnostics: applications in oral cancer," Nano Lett., vol. 5, no. 5, pp. 829-834, May 2005.

[5] S. Her, D. A. Jaffray, and C. Allen, "Gold nanoparticles for applications in cancer radiotherapy: Mechanisms and recent advancements," Adv. Drug Deliv. Rev., vol. 109, pp. 84-101, Jan. 2017.

[6] J. L. Su et al., "Advances in clinical and biomedical applications of photoacoustic imaging," Expert Opin. Med. Diagn., vol. 4, no. 6, pp. 497-510, Nov. 2010.

[7] R. A. Sperling and W. J. Parak, "Surface modification, functionalization and bioconjugation of colloidal inorganic nanoparticles," Philos. Trans. R. Soc. Lond. Math. Phys. Eng. Sci., vol. 368, no. 1915, pp. 1333-1383, Mar. 2010.

[8] J. Chen et al., "Gold Nanocages: Bioconjugation and Their Potential Use as Optical Imaging Contrast Agents," Nano Lett., vol. 5, no. 3, pp. 473-477, Mar. 2005.

[9] A. K. Oyelere, P. C. Chen, X. Huang, I. H. El-Sayed, and M. A. El-Sayed, "PeptideConjugated Gold Nanorods for Nuclear Targeting," Bioconjug. Chem., vol. 18, no. 5, pp. 1490-1497, Sep. 2007.

[10] M. Thomas and A. M. Klibanov, "Conjugation to gold nanoparticles enhances polyethylenimine's transfer of plasmid DNA into mammalian cells," Proc. Natl. Acad. Sci., vol. 100, no. 16, pp. 9138-9143, Aug. 2003.

[11] X. Huang, I. H. El-Sayed, W. Qian, and M. A. El-Sayed, "Cancer Cell Imaging and Photothermal Therapy in the Near-Infrared Region by Using Gold Nanorods," J. Am. Chem. Soc., vol. 128, no. 6, pp. 2115-2120, Feb. 2006.

[12] R. Tivony, L. Larush, O. Sela-Tavor, and S. Magdassi, "Biomedical imaging of colorectal cancer by near infrared fluorescent nanoparticles," J. Biomed. Nanotechnol., vol. 10, no. 6, pp. 1041-1048, Jun. 2014.

[13] N. J. Durr, T. Larson, D. K. Smith, B. A. Korgel, K. Sokolov, and A. Ben-Yakar, "TwoPhoton Luminescence Imaging of Cancer Cells Using Molecularly Targeted Gold Nanorods," Nano Lett., vol. 7, no. 4, pp. 941-945, Apr. 2007.

[14] "NanoHybrids - Top Nanoparticle Labels for Lateral Flow Assays." [Online]. Available: https://nanohybrids.net/pages/gold-nanoparticles-for-lateral-flow-assays. [Accessed: 03-Jul2017].

[15] J. Du, B. Zhu, and X. Chen, "Urine for Plasmonic Nanoparticle-Based Colorimetric Detection of Mercury Ion," Small, vol. 9, no. 24, pp. 4104-4111, Dec. 2013.

[16] T. Zheng et al., "Gold Nanoparticle-Enabled Blood Test for Early Stage Cancer Detection and Risk Assessment," ACS Appl. Mater. Interfaces, vol. 7, no. 12, pp. 6819-6827, Apr. 2015. 
[17] S. Y. Emelianov, P.-C. Li, and M. O'Donnell, "Photoacoustics for molecular imaging and therapy," Phys. Today, vol. 62, no. 8, p. 34, 2009.

[18] L. V. Wang and S. Hu, "Photoacoustic tomography: in vivo imaging from organelles to organs," Science, vol. 335, no. 6075, pp. 1458-1462, Mar. 2012.

[19] S. Y. Nam, L. M. Ricles, L. J. Suggs, and S. Y. Emelianov, "In vivo Ultrasound and Photoacoustic Monitoring of Mesenchymal Stem Cells Labeled with Gold Nanotracers," PLoS ONE, vol. 7, no. 5, p. e37267, May 2012.

[20] D. Yohan and B. D. Chithrani, "Applications of Nanoparticles in Nanomedicine," $J$. Biomed. Nanotechnol., vol. 10, no. 9, pp. 2371-2392, Sep. 2014.

[21] M. R. Detty, S. L. Gibson, and S. J. Wagner, "Current clinical and preclinical photosensitizers for use in photodynamic therapy," J. Med. Chem., vol. 47, no. 16, pp. 3897-3915, Jul. 2004.

[22] A. J. Gomes, L. O. Lunardi, J. M. Marchetti, C. N. Lunardi, and A. C. Tedesco, "Photobiological and ultrastructural studies of nanoparticles of poly(lactic-co-glycolic acid)-containing bacteriochlorophyll-a as a photosensitizer useful for PDT treatment," Drug Deliv., vol. 12, no. 3, pp. 159-164, Jun. 2005.

[23] S. Cui et al., "In Vivo Targeted Deep-Tissue Photodynamic Therapy Based on NearInfrared Light Triggered Upconversion Nanoconstruct," ACS Nano, vol. 7, no. 1, pp. 676688, Jan. 2013.

[24] Y. Wang et al., "Comparison Study of Gold Nanohexapods, Nanorods, and Nanocages for Photothermal Cancer Treatment," ACS Nano, vol. 7, no. 3, pp. 2068-2077, Mar. 2013.

[25] H. Matsudaira, A. M. Ueno, and I. Furuno, "Iodine contrast medium sensitizes cultured mammalian cells to X rays but not to gamma rays," Radiat. Res., vol. 84, no. 1, pp. 144 148, Oct. 1980.

[26] J. F. Hainfeld, D. N. Slatkin, and H. M. Smilowitz, "The use of gold nanoparticles to enhance radiotherapy in mice," Phys. Med. Biol., vol. 49, no. 18, pp. N309-N315, Sep. 2004.

[27] C. Yang, J. Uertz, D. Yohan, and B. D. Chithrani, "Peptide modified gold nanoparticles for improved cellular uptake, nuclear transport, and intracellular retention," Nanoscale, vol. 6, no. 20, pp. 12026-12033, Sep. 2014.

[28] D. B. Chithrani et al., "Gold nanoparticles as radiation sensitizers in cancer therapy," Radiat. Res., vol. 173, no. 6, pp. 719-728, Jun. 2010.

[29] P. Rai et al., "Development and applications of photo-triggered theranostic agents," $A d v$. Drug Deliv. Rev., vol. 62, no. 11, pp. 1094-1124, Aug. 2010.

[30] F.-Y. Cheng, C.-H. Su, P.-C. Wu, and C.-S. Yeh, "Multifunctional polymeric nanoparticles for combined chemotherapeutic and near-infrared photothermal cancer therapy in vitro and in vivo," Chem. Commun., vol. 46, no. 18, pp. 3167-3169, Apr. 2010.

[31] T. K. Jain, J. Richey, M. Strand, D. L. Leslie-Pelecky, C. Flask, and V. Labhasetwar, "Magnetic Nanoparticles with Dual Functional Properties: Drug Delivery and Magnetic Resonance Imaging," Biomaterials, vol. 29, no. 29, pp. 4012-4021, Oct. 2008.

[32] R. A. Petros and J. M. DeSimone, "Strategies in the design of nanoparticles for therapeutic applications," Nat. Rev. Drug Discov., vol. 9, no. 8, pp. 615-627, Aug. 2010.

[33] L. M. Bareford and P. W. Swaan, "Endocytic mechanisms for targeted drug delivery," $A d v$. Drug Deliv. Rev., vol. 59, no. 8, pp. 748-758, Aug. 2007. 
[34] B. D. Chithrani and W. C. W. Chan, "Elucidating the Mechanism of Cellular Uptake and Removal of Protein-Coated Gold Nanoparticles of Different Sizes and Shapes," Nano Lett., vol. 7, no. 6, pp. 1542-1550, Jun. 2007.

[35] P. Ghosh, G. Han, M. De, C. K. Kim, and V. M. Rotello, "Gold nanoparticles in delivery applications," Adv. Drug Deliv. Rev., vol. 60, no. 11, pp. 1307-1315, Aug. 2008.

[36] D. B. Chithrani, "Intracellular uptake, transport, and processing of gold nanostructures," Mol. Membr. Biol., vol. 27, no. 7, pp. 299-311, Oct. 2010.

[37] B. D. Chithrani, J. Stewart, C. Allen, and D. A. Jaffray, "Intracellular uptake, transport, and processing of nanostructures in cancer cells," Nanomedicine Nanotechnol. Biol. Med., vol. 5, no. 2, pp. 118-127, Jun. 2009.

[38] A. G. Tkachenko et al., "Cellular Trajectories of Peptide-Modified Gold Particle Complexes: Comparison of Nuclear Localization Signals and Peptide Transduction Domains," Bioconjug. Chem., vol. 15, no. 3, pp. 482-490, May 2004.

[39] P. Nativo, I. A. Prior, and M. Brust, "Uptake and Intracellular Fate of Surface-Modified Gold Nanoparticles," ACS Nano, vol. 2, no. 8, pp. 1639-1644, Aug. 2008.

[40] C. Yang, M. Neshatian, M. van Prooijen, and D. B. Chithrani, "Cancer Nanotechnology: Enhanced Therapeutic Response Using Peptide-Modified Gold Nanoparticles," J. Nanosci. Nanotechnol., vol. 14, no. 7, pp. 4813-4819, Jul. 2014.

[41] C. J. Yang and D. B. Chithrani, "Therapeutic Enhancement with Nuclear Targeted Gold Nanoparticles," Biophys. J., vol. 110, no. 3, p. 502a, Feb. 2016.

[42] S. Wilhelm et al., "Analysis of nanoparticle delivery to tumours," Nat. Rev. Mater., vol. 1, no. 5, p. 16014, Apr. 2016.

[43] J. Lazarovits, Y. Yang Chen, E. A. Sykes, and W. C. W. Chan, "Nanoparticle-blood interactions: the implications on solid tumour targeting," Chem. Commun., vol. 51, no. 14, pp. 2756-2767, 2015.

[44] Y. H. Bae and K. Park, "Targeted drug delivery to tumors: myths, reality and possibility," J. Control. Release Off. J. Control. Release Soc., vol. 153, no. 3, pp. 198-205, Aug. 2011.

[45] V. Mirshafiee, M. Mahmoudi, K. Lou, J. Cheng, and M. L. Kraft, "Protein corona significantly reduces active targeting yield," Chem. Commun. Camb. Engl., vol. 49, no. 25, pp. 2557-2559, Mar. 2013.

[46] E. A. Sykes, J. Chen, G. Zheng, and W. C. W. Chan, "Investigating the Impact of Nanoparticle Size on Active and Passive Tumor Targeting Efficiency," ACS Nano, vol. 8, no. 6, pp. 5696-5706, Jun. 2014.

[47] S. Sindhwani et al., "Three-Dimensional Optical Mapping of Nanoparticle Distribution in Intact Tissues," ACS Nano, vol. 10, no. 5, pp. 5468-5478, May 2016.

[48] E. A. Sykes et al., "Tailoring nanoparticle designs to target cancer based on tumor pathophysiology," Proc. Natl. Acad. Sci., vol. 113, no. 9, pp. E1142-E1151, Mar. 2016.

[49] A. Montaser, Inductively Coupled Plasma Mass Spectrometry. John Wiley \& Sons, 1998.

[50] N. H. Bings, A. Bogaerts, and J. A. C. Broekaert, "Atomic Spectroscopy," Anal. Chem., vol. 78, no. 12, pp. 3917-3946, Jun. 2006.

[51] J. Mora, S. Maestre, V. Hernandis, and J. L. Todolí, "Liquid-sample introduction in plasma spectrometry," TrAC Trends Anal. Chem., vol. 22, no. 3, pp. 123-132, Mar. 2003.

[52] G. Zachariadis, Inductively Coupled Plasma Atomic Emission Spectrometry: A Model Multi-Elemental Technique for Modern Analytical Laboratory, UK ed. edition. Hauppauge, N.Y: Nova Science Pub Inc, 2012. 
[53] J. S. Becker, "Trace and ultratrace analysis in liquids by atomic spectrometry," $\operatorname{Tr} A C$ Trends Anal. Chem., vol. 24, no. 3, pp. 243-254, Mar. 2005.

[54] P. W. J. M. Boumans, "Inductively coupled plasma emission spectroscopy. Part II: applications and fundamentals. Volume 2," 1987.

[55] R. Thomas, Practical Guide to ICP-MS: A Tutorial for Beginners, Third Edition, 3 edition. Boca Raton: CRC Press, 2013.

[56] D. Beauchemin, "Inductively Coupled Plasma Mass Spectrometry," Anal. Chem., vol. 82, no. 12, pp. 4786-4810, Jun. 2010.

[57] R. S. Houk and J. J. Thompson, "Inductively coupled plasma mass spectrometry," Mass Spectrom. Rev., vol. 7, no. 4, pp. 425-461, Jul. 1988.

[58] Concordia College, "Simultaneous Determination of Manganese and Nickel in Steel by Inductively Coupled Plasma Atomic Emission Spectrometry - Analytical Chemistry Lab Manual." [Online]. Available: http://sites.cord.edu/chem-330-lab-manual/experiments/icpaes. [Accessed: 08-Jul-2017].

[59] J. Marshall, S. Chenery, E. H. Evans, and A. Fisher, "Atomic Spectrometry Update-Atomic emission spectrometry," J. Anal. At. Spectrom., vol. 13, no. 6, p. 107R-130R, Jan. 1998.

[60] S. M. Nelms, Ed., Inductively Coupled Plasma Mass Spectrometry Handbook. Blackwell Publishing Ltd., 2005.

[61] A. J. Bard, "Fundamentals of analytical chemistry (Skoog, Douglas A.; West, Donald M.)," J. Chem. Educ., vol. 40, no. 11, p. 614, Nov. 1963.

[62] A Handbook of Silicate Rock Analysis | P.J. Potts | Springer. .

[63] J. R. Dean, Practical Inductively Coupled Plasma Spectroscopy. John Wiley \& Sons, Ltd, 2005.

[64] S. Böhme et al., "Quantification of A12O3 nanoparticles in human cell lines applying inductively coupled plasma mass spectrometry (neb-ICP-MS, LA-ICP-MS) and flow cytometry-based methods," J. Nanoparticle Res., vol. 16, no. 9, p. 2592, Sep. 2014.

[65] E. H. Wood, "A Low Temperature Wet Ashing Method Applied to the Study of the Electrolyte Composition of the Ventricular Musculature and Lung Parenchyma of the Dog," J. Biol. Chem., vol. 143, no. 1, pp. 165-170, Mar. 1942.

[66] R. V. Grieken and A. Markowicz, Eds., Handbook of X-Ray Spectrometry, Second Edition, 2 edition. New York: CRC Press, 2001.

[67] R. Klockenkämper and A. von Bohlen, Total-Reflection X-Ray Fluorescence Analysis and Related Methods. John Wiley \& Sons, 2014.

[68] International Union of Pure and Applied Chemistry, "Nomenclature system for x-ray spectroscopy," Pure Appl Chern, vol. 63, no. 5, pp. 735-746, 1991.

[69] P. Wobrauschek and H. Aiginger, "Total-Reflection X-Ray Fluorescence Spectrometric Determination of Elements in Nanogram Amounts," Anal. Chem., vol. 47, no. 6, 1975.

[70] X. Huang and M. A. El-Sayed, "Gold nanoparticles: Optical properties and implementations in cancer diagnosis and photothermal therapy," J. Adv. Res., vol. 1, no. 1, pp. 13-28, Jan. 2010.

[71] J. F. Hainfeld, F. A. Dilmanian, D. N. Slatkin, and H. M. Smilowitz, "Radiotherapy enhancement with gold nanoparticles," J. Pharm. Pharmacol., vol. 60, no. 8, pp. 977-985, Aug. 2008.

[72] R. Allabashi, W. Stach, A. de la Escosura-Muñiz, L. Liste-Calleja, and A. Merkoçi, "ICPMS: a powerful technique for quantitative determination of gold nanoparticles without previous dissolving," J. Nanoparticle Res., vol. 11, no. 8, p. 2003, Nov. 2009. 
[73] D. Vanhecke, L. Rodriguez-Lorenzo, M. J. D. Clift, F. Blank, A. Petri-Fink, and B. RothenRutishauser, "Quantification of nanoparticles at the single-cell level: an overview about state-of-the-art techniques and their limitations," Nanomed., vol. 9, no. 12, pp. 1885-1900, 2014.

[74] A.-L. Fabricius, L. Duester, B. Meermann, and T. A. Ternes, "ICP-MS-based characterization of inorganic nanoparticles--sample preparation and off-line fractionation strategies," Anal. Bioanal. Chem., vol. 406, no. 2, pp. 467-479, Jan. 2014.

[75] Y. Yoneda and T. Horiuchi, "Optical Flats for Use in X-Ray Spectrochemical Microanalysis,” Rev. Sci. Instrum., vol. 42, no. 7, pp. 1069-1070, Jul. 1971.

[76] N. V. Alov, "Total reflection X-ray fluorescence analysis: Physical foundations and analytical application (A review)," Inorg. Mater., vol. 47, no. 14, pp. 1487-1499, Dec. 2011.

[77] R. Klockenkämper and A. von Bohlen, "Elemental Analysis of Environmental Samples by Total Reflection X-Ray Fluorescence: a Review," X-Ray Spectrom., vol. 25, no. 4, pp. 156162, Jul. 1996.

[78] L. M. Marcó P. and E. A. Hernández-Caraballo, "Direct analysis of biological samples by total reflection X-ray fluorescence," Spectrochim. Acta Part B At. Spectrosc., vol. 59, no. 8, pp. 1077-1090, Aug. 2004.

[79] V. A. Solé, E. Papillon, M. Cotte, P. Walter, and J. Susini, "A multiplatform code for the analysis of energy-dispersive X-ray fluorescence spectra," Spectrochim. Acta Part B At. Spectrosc., vol. 62, no. 1, pp. 63-68, Jan. 2007.

[80] J. Ermer and J. H. M. Miller, Eds., Method Validation in Pharmaceutical Analysis: A Guide to Best Practice, 1 edition. Weinheim: Wiley-VCH, 2005.

[81] G. Gauglitz and D. S. Moore, Eds., Handbook of Spectroscopy, 2 edition. Weinheim, Germany: Wiley-VCH, 2014.

[82] D. Dale, "PyMCA Documentation: Getting Started tutorial," 25-Sep-2008. [Online]. Available: http://pymca.sourceforge.net/PyMcaCHESS.pdf. [Accessed: 08-Aug-2017].

[83] A. Albanese and W. C. W. Chan, "Effect of Gold Nanoparticle Aggregation on Cell Uptake and Toxicity," ACS Nano, vol. 5, no. 7, pp. 5478-5489, Jul. 2011.

[84] P. Pengo, L. Pasquato, and P. Scrimin, "Gold nanoparticles protected with triethyleneglycol-Functionalized thiolates: acid-Induced clustering of the aggregates and solvent dependent optical properties," J. Supramol. Chem., vol. 2, no. 1, pp. 305-310, Jan. 2002.

[85] J. B. Carroll, B. L. Frankamp, S. Srivastava, and V. M. Rotello, "Electrostatic self-assembly of structured gold nanoparticle/polyhedral oligomeric silsesquioxane (POSS) nanocomposites," J. Mater. Chem., vol. 14, no. 4, pp. 690-694, Feb. 2004.

[86] Z. Peng, T. Walther, and K. Kleinermanns, "Influence of Intense Pulsed Laser Irradiation on Optical and Morphological Properties of Gold Nanoparticle Aggregates Produced by Surface Acid-Base Reactions," Langmuir, vol. 21, no. 10, pp. 4249-4253, May 2005.

[87] J. Moore and C. Stanitski, Chemistry: The Molecular Science, 5 edition. Stamford, CT: Brooks Cole, 2014.

[88] G. P. Robertson, D. C. Coleman, C. S. Bledsoe, and P. Sollins, Eds., Standard Soil Methods for Long-Term Ecological Research. New York: Oxford University Press, 1999.

[89] B. D. Chithrani, A. A. Ghazani, and W. C. W. Chan, "Determining the Size and Shape Dependence of Gold Nanoparticle Uptake into Mammalian Cells," Nano Lett., vol. 6, no. 4, pp. 662-668, Apr. 2006. 
[90] D. Yohan, C. Cruje, X. Lu, and D. Chithrani, "Elucidating the Uptake and Distribution of Nanoparticles in Solid Tumors via a Multilayered Cell Culture Model," Nano-Micro Lett., vol. 7, no. 2, pp. 127-137, Apr. 2015.

[91] H. C. Fischer, S. Fournier-Bidoz, W. C. W. Chan, and K. S. Pang, "Quantitative detection of engineered nanoparticles in tissues and organs: An investigation of efficacy and linear dynamic ranges using ICP-AES," NanoBiotechnology, vol. 3, no. 1, pp. 46-54, Mar. 2007.

[92] E. Porcel et al., "Platinum nanoparticles: a promising material for future cancer therapy?," Nanotechnology, vol. 21, no. 8, p. 85103, Feb. 2010.

[93] S. Hrapovic, Y. Liu, K. B. Male, and J. H. T. Luong, "Electrochemical Biosensing Platforms Using Platinum Nanoparticles and Carbon Nanotubes," Anal. Chem., vol. 76, no. 4, pp. 1083-1088, Feb. 2004.

[94] D. E. Sosnovik, M. Nahrendorf, and R. Weissleder, "Magnetic nanoparticles for MR imaging: agents, techniques and cardiovascular applications," Basic Res. Cardiol., vol. 103, no. 2, pp. 122-130, Mar. 2008.

[95] B. T. Farrell et al., "Using iron oxide nanoparticles to diagnose CNS inflammatory diseases and PCNSL," Neurology, vol. 81, no. 3, pp. 256-263, Jul. 2013.

[96] L. Maggiorella et al., "Nanoscale radiotherapy with hafnium oxide nanoparticles," Future Oncol. Lond. Engl., vol. 8, no. 9, pp. 1167-1181, Sep. 2012.

[97] J. G. Mendoza et al., "Synthesis and characterization of hafnium oxide films for thermo and photoluminescence applications," Appl. Radiat. Isot. Data Instrum. Methods Use Agric. Ind. Med., vol. 68, no. 4-5, pp. 696-699, May 2010.

[98] D. K. Chatterjee, L. S. Fong, and Y. Zhang, "Nanoparticles in photodynamic therapy: an emerging paradigm," Adv. Drug Deliv. Rev., vol. 60, no. 15, pp. 1627-1637, Dec. 2008.

[99] H. Gong, R. Peng, and Z. Liu, "Carbon nanotubes for biomedical imaging: The recent advances," Adv. Drug Deliv. Rev., vol. 65, no. 15, pp. 1951-1963, Dec. 2013.

[100] M. Chakrabarti, R. Kiseleva, A. Vertegel, and S. K. Ray, "Carbon Nanomaterials for Drug Delivery and Cancer Therapy,” J. Nanosci. Nanotechnol., vol. 15, no. 8, pp. 55015511, Aug. 2015.

[101] H. Dumortier et al., "Functionalized Carbon Nanotubes Are Non-Cytotoxic and Preserve the Functionality of Primary Immune Cells," Nano Lett., vol. 6, no. 7, pp. 1522-1528, Jul. 2006.

[102] K. Geraki, M. J. Farquharson, and D. A. Bradley, "X-ray fluorescence and energy dispersive x-ray diffraction for the quantification of elemental concentrations in breast tissue," Phys. Med. Biol., vol. 49, no. 1, pp. 99-110, Jan. 2004.

[103] E. L. Crossley, J. B. Aitken, S. Vogt, H. H. Harris, and L. M. Rendina, "Uptake and Distribution of a Platinum(ii)-Carborane Complex Within a Tumour Cell Using Synchrotron XRF Imaging," Aust. J. Chem., vol. 64, no. 3, pp. 253-257, Apr. 2011.

[104] K. Huang et al., "Size-Dependent Localization and Penetration of Ultrasmall Gold Nanoparticles in Cancer Cells, Multicellular Spheroids, and Tumors in Vivo," ACS Nano, vol. 6, no. 5, pp. 4483-4493, May 2012.

[105] N. Lewinski, V. Colvin, and R. Drezek, "Cytotoxicity of nanoparticles," Small Weinh. Bergstr. Ger., vol. 4, no. 1, pp. 26-49, Jan. 2008.

[106] A. D. Maynard and R. J. Aitken, "Safe handling of nanotechnology' ten years on," Nat. Nanotechnol., vol. 11, no. 12, pp. 998-1000, Dec. 2016. 